\title{
5 Zur Kombinierbarkeit von Nomen und Verb
}

\subsection{Einleitendes}

Was die Kombinationsmöglichkeiten von Verb und Nomen in einem FVG angeht, so deutet zunächst einiges darauf hin, dass die Besetzung der NP- bzw. PP-Position eines FVG vollkommen arbiträr ist. Als Beleg für die Beliebigkeit der Kombination lässt sich mit Krenn/Erbach (1994: 379f.) das unterschiedliche Verhalten der bedeutungsnahen Nomina Beschluss und Entscheidung in FVG mit treffen bzw. fassen anführen:
a. eine Entscheidung treffen
b. eine Entscheidung fassen
c. *einen Beschluss treffen
e. einen Besuch machen/'tun

Da Beschluss und Entscheidung als Quasi-Synonyme gelten müssen, bleibt es unerklärt und wohl auch unerklärbar, weshalb Entscheidung mit fassen und treffen (mit Letzterem wohl etwas besser als mit Ersterem) kombiniert werden kann, während Beschluss nur mit fassen und nicht mit treffen kompatibel ist. Hier wird man annehmen müssen, dass die Kombination von Verb und NP im Lexikon festgeschrieben ist.

Es gibt allerdings auch Beispiele dafür, dass die Selektion der Substantive nicht gänzlich beliebig ist, sondern nach semantischen Klassen erfolgt, $d$. h. nach Gruppen von Substantiven, die ein signifikantes Bedeutungsmerkmal teilen. Für eine solche Selektion lassen sich Verbindungen mit dem Verb begehen anführen (vgl. Sailer 2003: $260 \mathrm{zu}$ engl. commit). Dieses selektiert Nomina, die ein Verbrechen oder eine negativ bewertete Handlung bezeichnen:

(2) Fehler, Verbrechen, Mord, Totschlag, Einbruch, Diebstahl, Verkehrsdelikt, Überfall, Betrug

Eine Selektion der Nomina über semantische Klassen ist nach Krenn/Erbach auch bei den Verbindungen mit haben wirksam: „In the case of support verb constructions such as Angst/Probleme/Lust/Freude/Hoffnungen/Befürchtungen/Schwierigkeiten haben, selection of the predicative noun by semantic sorts 
suggests itself“ (Erbach/Krenn 1994: 379). Diese Einschätzung erweist sich jedoch bei näherem Hinsehen als zu optimistisch, da unklar bleibt, weshalb nur bestimmte Lexeme der entsprechenden semantischen Klasse MENTALE ZUSTÄNDE selektiert werden, während bei anderen keine Verbindung mit haben möglich ist, vgl. (3).

*Bestürzung haben, *Traurigkeit haben, *Verzweiflung haben, *Liebe haben ...

Angesichts dieser Situation könnte eine Lösung sein, für FVG mit haben eine Liste mit den jeweils einschlägigen Lexemen zu postulieren. Eine solche Liste würde dann mindestens die Beispiele in (4) umfassen. ${ }^{84}$

(4) die Absicht, Achtung (vor), eine Ahnung, Angst, eine Anwandlung, die Befürchtung, die/eine Begabung, eine Beobachtungsgabe, das Bestreben, Bedenken, ein Bewusstsein (von), Durst, Freude, Furcht, Gefallen, das/ein Gefühl, die Gewissheit, die/eine Eigenschaft, eine Einstellung, die/eine Empfindung, die/eine Erkenntnis, die Erwartung, das/ein Gespür, den Glauben, einen Hass, die Hoffnung, Hunger, Interesse, Kenntnis, Kummer, Langeweile, die/eine Meinung, Mühe, den Mut, Panik, einen Plan, Respekt, Skrupel, Skepsis, Spaß, den/einen Traum, die Überzeugung, den/einen Verdacht, die/eine Vermutung, Verständnis, Vertrauen, die/eine Vorstellung, den Wahn, den/einen Wunsch, Zutrauen, Zweifel

Umfangreiche und arbiträr zusammengestellte Lexemlisten wie diese sind jedoch in kognitiver Hinsicht wenig plausibel, so dass diese Annahme nicht attraktiv ist.

Verschärft stellt sich das angedeutete Problem bei den Verbindungen mit kommen und bringen. Während im Fall von haben mit der Klasse MENTALE ZUSTÄNDE immerhin noch eine relativ gut konturierte Gruppe von Nomina als Reservoir für die auftretenden Nominale benannt werden konnte, ist bei den mit bringen verbindbaren Nomina keine semantische Klasse auf der Basis eines gemeinsamen Inhaltsmerkmals konstruierbar, vgl. die Auflistung in (5): ${ }^{85}$

84 Zum Artikelgebrauch finden sich in der Liste finden nur vorläufige Angaben; Weiteres in Kapitel 6.3.

85 Der Bestand ergibt sich aus einer Sammlung der in der Forschungsliteratur genannten Fälle sowie weiterer Einzelfunde. Eine systematische Suche (etwa nach Kookkurrenzen mit bringen 
(5) Abbruch, Abschluss, Anklage, Anzeige, Anwendung, Aufführung, Ausführung, Austragung, Ausdruck, Darstellung, Darbietung, Druck, Durchführung, Einsatz, Entscheidung, Umsetzung, Verbreitung, Verkauf, Verlesung, Veröffentlichung, Verteilung, Versteigerung, Vollendung, Vorführung, Vortrag ${ }^{86}$

Anders als bei den Substantiven in (4) ist z. B. zwischen Abbruch, Anklage, Druck und Versteigerung zumindest auf Anhieb keine inhaltliche Übereinstimmung zu erkennen. In den folgenden Abschnitten soll untersucht werden, ob dennoch Regularitäten bzw. Beschränkungen erkennbar sind, die die Selektion der Nomina in einem FVG steuern, oder ob hier tatsächlich, wie die Nomina in (5) suggerieren, von einer beliebigen Auswahl auszugehen ist. Im erstgenannten Fall wären FVG als produktives Muster anzusehen, im zweiten Fall hätte man von einer Liste einzelner Lexikoneinträge auszugehen.

Der Fragestellung entsprechend werden im Weiteren die Fügungen in den Blick genommen, in denen eine größere Anzahl von Nomina auftritt. Dies betrifft neben den FVG mit haben und bringen auch die Verbindungen mit geben und machen. ${ }^{87}$ Bei FVG wie eine Entscheidung treffen oder einen Beschluss fassen, die gar keine oder nur wenige parallele Bildungen aufweisen, stellt sich die Frage nach der Produktivität naturgemäß nicht. FVG mit bringen, geben, haben und machen sind zudem diejenigen, die in der bisherigen Forschung das größte Interesse

zur/zum) ergibt zu wenig einschlägige Ergebnisse. Die der Forschungsliteratur entnommenen Fälle wurden sämtlich an der COSMAS II-Datenbank (Archiv der geschriebenen Sprache) überprüft. Verbindungen, die sich nicht durch COSMAS belegen ließen, wurden aus der Liste in (5) ausgeschlossen, so z. B. das häufig zitierte, von Karl Kraus in einer Sprachglosse inkriminierte zur Abschaltung bringen ,abschalten'.

86 Einige wenige der FVG mit den genannten Nomina haben ein Kausativum neben sich (s. Kapitel 2.3.1). Kausativ ist z. B.: „Mit waghalsigen Störmanövern auf hoher See hat die Tierschutzgruppe Sea Shepherd die japanischen Walfänger zum Abbruch der Jagdsaison im Südpolarmeer gebracht“ (Nürnberger Zeitung, 19.02.2011, S. 24; Zugriff 06.04.2021). Hier veranlasst ein Agens einen anderen Agens, eine Handlung abzubrechen. Eine Fügung mit implikativem Bedeutungsaufbau liegt hingegen im folgenden Satz vor: „Als Möthrath gestern acht neue Beweisanträge stellte und vier weitere ankündigte, war es mit der Geduld des Vorsitzenden Richters, Rolf-Rainer Nebe, zu Ende: ,Die Anträge dienen nur dem Zwecke der Prozessverschleppung.' Ziel sei es, das Verfahren zum Abbruch zu bringen, um einer möglichen Verurteilung vorerst zu entgehen“ (Rhein-Zeitung, 21.04.2006; Zugriff 06.04.2021).

87 FVG mit kommen sind zwar ebenfalls häufig. Dabei handelt es sich aber meist lediglich um die passive Version der aktiven FVG mit bringen (zur Anwendung bringen/kommen). Da die kommen-FVG offensichtlich von den bringen-FVG abhängen, ist die Frage nach der Selektion der Nomina gegenstandslos. 
gefunden haben (dies gilt besonders für die Fügungen mit bringen). Dass die genannten Bildungen hier im Mittelpunkt stehen, ist auch aus diesem Grund angebracht.

\subsection{FVG mit bringen (zu)}

\subsubsection{Der Vorschlag von Winhart (2005)}

Die hier zu beantwortende Frage nach möglichen Selektionskriterien für die Substantive in FVG ist von der Forschung bislang eher stiefmütterlich behandelt worden - bei der Vielzahl der Studien zum Thema FVG ist dies ein überraschender Befund. Eine Ausnahme stellt die Untersuchung von Winhart (2005) dar, in der erstmals eine Auseinandersetzung mit dem Selektionsproblem erfolgt ist. Auf diese Arbeit sei hier deshalb ausführlicher eingegangen. Winhart vertritt die Hypothese, dass die Kombination von Verb und Nominalisierung nicht beliebig sei; vielmehr könne die ,enge Verbindung zwischen Verb und Nominalisierung [...] auf die (partielle) Übereinstimmung der lexikalisch-semantischen Strukturen zurückgeführt werden“ (Winhart 2005: 194). Diese Übereinstimmung der Strukturen habe zur Folge, dass die Argumente der Nominalisierung - bzw. des der Nominalisierung zugrunde liegenden Verbs - als Argumente des Funktionsverbs interpretiert werden könnten (Winhart 2005: 157). Winharts These, dass die lexikalisch-semantischen Strukuren von Verb und Nomen eines FVG enge Parallelen aufweisen und auf dieser Basis die Beschränkungen in der Besetzung der N-Position erklärbar sind, sei auch für dieses Kapitel zugrunde gelegt.

Im Hinblick auf die FVG mit bringen, die hier zunächst als Beispiel herausgegriffen seien, ${ }^{88}$ nennt Winhart (2005) die folgenden drei wesentlichen Gesichtspunkte, die die Kombinierbarkeit von Verb und Nomen begründen. Als erste Beschränkung gilt nach Winhart:

Da bringen den Übergang von einem Zustand in einen anderen Zustand explizit macht, ist eine grundlegende Bedingung, dass das Objekt, das der Veränderung unterworfen wird, bereits vorher existiert.

(Winhart 2005: 161).

Als zweite Einschränkung wird angeführt, dass bringen nur mit solchen Nomina verbunden werden kann, die einen sog. „Resultatszustand“ kodieren (Winhart

$88 \mathrm{Zu}$ diesen zählt Winhart im Anschluss an Herrlitz (1973: 160ff.) eine große Zahl von Fügungen, die nicht als FVG im Sinne dieser Untersuchung gelten. 
2005: 157-161). Den Begriff „Resultatszustand“ bzw. „Resultatszustandsnominalisierung“ („RZ-NOM“) entnimmt Winhart der Untersuchung von Ehrich/Rapp (2000) zur Argumentstruktur von Nominalisierungen. Laut Ehrich/Rapp (2000: 251-253) sind damit Zustände gemeint, die durch ein vorausgehendes Ereignis verursacht sind, vgl. (6).

(6) a. die jahrelange Absperrung des Geländes

b. Die Verunreinigung des Flusses besteht fort

c. Die Beklebung der Wand besteht unverändert fort.

(Beispiele Ehrich/Rapp 2000: 252f.)

Als Test für das Vorliegen einer Resultatszustandsnominalisierung schlagen Ehrich/Rapp (2000: 252) die Kombinierbarkeit mit sog. „stationären Prädikaten“ wie vorliegend, bestehend $u$. dgl. vor, vgl. neben den Beispielen in (6) auch die folgenden Verbindungen:

(7) a. Die bestehende Absperrung des Geländes/Verunreinigung des Flusses

b. Die vorgefundene/kartographisch registrierte Absperrung des Geländes/Verunreinigung des Flusses

Als dritte Bedingung wird schließlich formuliert:

Außerdem muss, da die Nominalisierung den Resultatszustand herausgreift, dieser auch beim zugrunde liegenden Verb lexikalisch betonter sein als die Tätigkeitsphase, die zu diesem Endzustand führt.

(Winhart 2005: 161)

Die drei von Winhart postulierten Beschränkungen - Vorexistenz des Objekts, Vorliegen einer Resultatszustandsnominalisierung, Betonung des Endzustandes, - seien im Folgenden in umgekehrter Reihenfolge diskutiert, da das erste Kriterium eine wesentlich ausführlichere Behandlung erfordert als die beiden zuletzt genannten.

Zur These, dass es sich bei den Nominalen in Verbindungen mit bringen $(z u)$ um Resultatszustandsnominalisierungen handle, ist anzumerken, dass der von Ehrich/Rapp (2000) vorgeschlagene Test - die Kombinierbarkeit mit stationären Prädikaten - auf die meisten der Nomina in (7) gerade nicht angewandt werden kann, vgl. die folgenden Beispiele: 
(8)
a. *Die Aufführung von ,Tannhäuser ${ }^{\star}$ besteht fort/`die bestehende Auf- führung von ,Tannhäuser
b. *Der Einsatz des Fußballspielers besteht fort./ ${ }^{\star}$ der bestehende Einsatz des Fußballspielers
c. ${ }^{\star}$ Die Vollendung des Domes besteht fort./?die bestehende Vollendung des Domes
d. *Die Versteigerung der Kunstwerke besteht fort./* die bestehende Ver- steigerung der Kunstwerke

Die Nomina in FVG mit bringen stellen somit - entgegen Winharts These - nicht generell Versprachlichungen eines Resultatszustands dar. ${ }^{89}$

Als zweites Kriterium für die in Fügungen mit bringen $z u$ auftretenden Nominalisierungen hatte Winhart die ,lexikalische Betonung' des zweiten Prädikatsteils der Verben genannt, die den betreffenden Nomina zugrunde liegen. Den Hintergrund dieser These bildet die Annahme, dass Accomplishments generell durch eine zweiteilige Ereignisstruktur gekennzeichnet sind: Sie enthalten eine unspezifische Tätigkeits-Phase („DO-Phase“) sowie einen Zustand, in welchen die Tätigkeit mündet (vgl. grundlegend Dowty 1979: 184). Diese zweiteilige Struktur kommt auch in der von Winhart genutzten Formalisierung zum Ausdruck, in welcher das BE-Prädikat mit einem den Zustand näher kennzeichnenden Subskript versehen wird:

$$
\text { vollenden: } \lambda \mathrm{y} \lambda \mathrm{x} \lambda \mathrm{e}\left[\mathrm{DO}((\mathrm{x}, \mathrm{y}) \mathrm{r}) \& \operatorname{BEC}\left(\left(\mathrm{BE}_{\text {vollendet }}((\mathrm{y}) \mathrm{s})\right) \mathrm{e}\right)\right]
$$

(Winhart 2005: 158)

Das Subskriptum „,vollendet“ in (9) - das im Übrigen nicht auf die sonst von Winhart benutzte Notation von Ehrich/Rapp (2000) zurückgeht, sondern von ihr selbst eingeführt wurde - soll hier offenbar die herausgehobene Rolle des BE-Prädikats auch in der Formalisierung deutlich machen. Auf der Grundlage einer zweiphasigen Ereignisstruktur wie in (9) formuliert Winhart die bereits angesprochene These etwas ausführlicher:

Grundlegend scheint außerdem zu sein, dass die den Nominalisierungen zugrunde liegenden Verben eine unspezifizierte Do-Phase haben und die dem einzelnen Verb eigene lexikalische Information im BE-Prädikat steckt.

(Winhart 2005: 158)

89 Dies liegt auch nicht an unterschiedlichen Klassenabgrenzungen, da die in dieser Untersuchung als FVG definierten Verbindungen ebenfalls FVG im Sinne von Winhart (2005) sind. 
In der prominenten Position des BE-Prädikats wird auch eine wesentliche Übereinstimmung des Basisverbs mit bringen gesehen. Dieses hat nach Winhart ebenfalls eine unspezifizierte DO-Phase und eine durch das Prädikat APPL (y, b $)^{90}$ spezifizierte Endphase, die die eigentliche semantische Information enthält, vgl. (10):

$$
\text { bringen: } \lambda y \lambda_{x} \lambda e[D O((x, y) r) \& \operatorname{BEC}((\operatorname{APPL}((y, a) \operatorname{APPL}(y, b) s)) e)]
$$

(Winhart 2002: 156, 158)

Die von Winhart postulierte lexikalische Betonung des zweites Ereignisteils, welche diese für eine Kombination mit bringen qualifizieren soll, bleibt jedoch insgesamt vage: So fehlt in der von Winhart angesetzten Notation der semantischen Struktur von bringen ein Subskriptum, das mit „BEvollendet (y)“ vergleichbar wäre. Es wird ferner nicht deutlich, ob es neben den betonten Resultatszuständen auch unbetonte gibt und nach welchen Kriterien beide zu unterscheiden wären. Was hier als ,lexikalische Betonung، bezeichnet wird, stellt vielmehr eine generelle Eigenschaft zweiphasiger Verben dar, gilt also für alle Verben mit Accomplishment- und Achievementstruktur. So ist etwa auch für das von Winhart (2005: 177) diskutierte verletzen eine unspezifische Tätigkeitsphase sowie eine spezifische, „lexikalisch betonte“ Endphase zu postulieren, die ganz im Sinne Winharts ebenfalls mit einem Subskript „verletzt“ gekennzeichnet werden könnte, vgl. (11).

verletzen: $\lambda \mathrm{y} \lambda \mathrm{x} \lambda \mathrm{e}\left[\mathrm{DO}((\mathrm{x}, \mathrm{y}) \mathrm{r}) \& \mathrm{BEC}\left(\left(\mathrm{BE}_{\text {verletzt }}((\mathrm{y}) \mathrm{s})\right) \mathrm{e}\right)\right]$

(vgl. Winhart 2005: 145, Ehrich/Rapp 2000: 262)

Sowohl verletzen als auch vollenden ist gemeinsam, dass $\mathrm{x}$ etwas tut, so dass ein Zustand von y eintritt. Dass im Fall von vollenden der Endzustand betonter, lexikalisch in irgend einer Weise gehaltvoller sei als der Endzustand von verletzen, ist nicht anzunehmen. Damit entfällt auch das zweite von Winhart genannte Kriterium für die Verbindung von Nominalisierungen mit bringen.

Eine Antwort auf die Frage, welche Faktoren die Wahl der Nominalisierungen in den FVG mit bringen beschränken, bietet möglicherweise jedoch das erste

90 Bei APPL handelt es sich nach Ehrich/Rapp (2000: 256) um ein zweistelliges Prädikat, das „besagt, dass das erste Argument (APPLIKATUM) dem zweiten Agument (RELATUM) zugeordnet ist“. Es entspricht dem sonst häufiger angesetzten Prädikat Loc bzw. BE AT (z. B. Jackendoff 2002) und wird diesem von Ehrich/Rapp vorgezogen, weil APPL deutlicher zum Ausdruck bringt, dass es sich nicht nur um räumliche Relationen handelt. 
von Winharts drei Kriterien, nämlich die „grundlegende Bedingung, dass das Objekt [des der Nominalisierung zugrundeliegenden Verbs, V. H.], das der Veränderung unterworfen wird, bereits vorher existiert“ (2005: 161). Diese Beschränkung illustriert die Autorin an der Verbindung zur Vollendung bringen; vgl. dazu zunächst Beispiel (12):

(12) Anfang des 19. Jahrhunderts entdeckte Boisserée die ursprünglichen Baupläne und setzte nun alles daran, den Dom zur Vollendung zu bringen.

(Beispiel Winhart 2005: 156)

Das Basisverb von Vollendung, so Winhart, zeichne sich durch ein „effiziertes Thema“ aus,

das vom Patiens nicht referentiell unabhängig ist. D.h. im obigen Beispiel, bevor der Baumeister den Dom vollendet, besteht bereits ein Zustand des Doms, der mit dem Endzustand referentiell identifiziert werden kann.

(Winhart 2005: 156)

Den Dom zur Vollendung bringen setzt, anders ausgedrückt, voraus, dass - trotz z. B. der noch nicht fertiggestellten Türme - bereits ein Objekt besteht, das als Dom bezeichnet werden kann und das in einem gewissen Maße auch bereits wesentliche Merkmale und Funktionen eines Domes besitzt (z. B. dass man Gottesdienste in ihm abhalten kann). Gleiches gilt auch für andere mögliche PatiensRollen von vollenden/zur Vollendung bringen: Wenn etwa ein Komponist eine Symphonie vollendet, dann sind bereits wesentliche Bestandteile der Symphonie vorhanden, die es erlauben, das Werk als Symphonie zu identifizieren; lediglich z. B. die Orchestrierung einzelner Teile oder vergleichbare kleinere Arbeiten fehlen noch.

Das Kriterium der Vorexistenz des Objekts kommt insofern auch in der von Winhart gewählten Formalisierung zum Ausdruck, als das y-Argument sowohl im DO-Prädikat als auch im BECOME-Prädikat in Erscheinung tritt:

$$
\text { vollenden: } \lambda y \lambda_{x} \lambda e[D O((x, y) r) \& \operatorname{BEC}((B E((y) s)) e)]
$$

(Winhart 2005: 156)

Mit der von Winhart herausgearbeiteten Vorexistenz des Objekts ist auch eine wesentliche Eigenschaft von bringen benannt: Wer etwas wohin bringt, erschafft das Objekt nicht neu, sondern vollzieht diese Handlung an einem bereits vorhandenen Patiens, vgl. dazu nochmals den von Winhart angesetzten Lexikoneintrag: 
(10')

$$
\text { bringen: } \lambda y \lambda_{x} \lambda e[\operatorname{DO}((x, y) r) \& \operatorname{BEC}((\operatorname{APPL}((y, a) \operatorname{APPL}(y, b) s)) e)]
$$

(Winhart 2005: 156)

In dem Vorhandensein des y sowohl im DO- als auch im BECOME-Prädikat besteht somit in der Tat eine „Übereinstimmung der lexikalisch-semantischen Strukturen“ (Winhart 2005: 194) zwischen bringen und vollenden/Vollendung, welche Verb und Nominalisierung aufeinander abbildbar macht.

\subsubsection{Offene Fragen}

Sieht man die Nominalisierungen in (5) durch, bestätigt sich Winharts These, dass allen Nominalisierungen ein Verb zugrunde liegt, dessen Patiens vor dem Ereignis existiert. Wenn man dies als eine Beschränkung auffasst, die bringen den Nominalisierungen im FVG auferlegt, so wird auch erklärbar, weshalb Verben bzw. Nominalisierungen, die die Herstellung eines Patiens implizieren, nicht in FVG mit bringen (zu) auftreten können, vgl. (14).

(14) a. produzieren: ${ }^{\star}$ Die Firma bringt Bleistifte zur Produktion.

b. erschaffen: ${ }^{\star}$ Der Alchimist bringt einen Homunculus zur Erschaffung.

c. destillieren: *Der Winzer bringt Weinbrand zur Destillation.

Um die mit bringen $(z u)$ kompatiblen Nominalisierungen näher zu bestimmen, sind allerdings noch weitere, über Winharts Vorexistenzkriterium hinausgehende Eigenschaften herauszuarbeiten; denn dass der Patiens vor dem Ereignis bereits besteht, ist eine Eigenschaft, die für eine sehr große Zahl von Verben gilt, ohne dass diese als Kandidaten für eine Verbindung mit bringen in Betracht kämen. Dazu gehören vor allem ornative Verben (bemalen, bespritzen, einölen; bei Ehrich/Rapp 2000: 288 „Applikativverben“) sowie die große Gruppe der sog. „Modifikations-“ und „Vernichtungsverben“ (renovieren, verletzen, vernichten; Ehrich/Rapp 2000: 262, 284), vgl. z. B. die folgenden Beispiele:

(15) a. bemalen: *Der Handwerker bringt eine Wand zur Bemalung.

b. renovieren: ${ }^{\star}$ Der Handwerker bringt ein Haus zur Renovierung.

c. verletzen: ^Das Auto bringt den Mann zur Verletzung.

d. verbrennen: \#Die Sekretärin bringt die Unterlagen zur Verbrennung.

Weshalb ornative Verben sowie Modifikations- und Vernichtungsverben nicht in einem FVG mit bringen ( $z u$ ) auftreten, wäre somit noch zu klären. 


\subsubsection{Zur Beschreibungssprache}

Um diese und ggf. noch weitere Beschränkungen bei der Besetzung der N-Position in den betreffenden FVG ermitteln zu können, soll im Folgenden versucht werden, die Semantik des Verbs bringen näher zu beleuchten. Sind die Bedeutungsstrukturen von bringen in ihren Grundzügen erfasst, wird im besten Fall auch klarer, welche Beschränkungen dieses Verb den Nominalisierungen auferlegt.

Für die Erfassung der Bedeutungsstrukturen von bringen bedarf es freilich einer hinreichend präzisen Beschreibungssprache. Die von Winhart (2005) angewandten prädikatenlogischen Dekompositionsstrukturen, die sich an Ehrich/ Rapp (2000) anlehnen und die letztlich im theoretischen Rahmen der Zwei-Ebenen-Semantik (Bierwisch 1983) zu verorten sind, erweisen sich offenkundig als zu grobkörnig, um die gesuchte Übereinstimmung der lexikalisch-semantischen Strukturen modellieren zu können. Die Grobkörnigkeit der Formalisierung zeigt sich unter anderem darin, dass die Partizipanten lediglich als „x“ bzw. „y“ notiert werden, ohne dass relevante Unterschiede wie der zwischen einem effizierten und einem affizierten Patiens darstellbar sind. Dies hat zur Folge, dass z. B. das in (15b) angesprochene „Modifikationsverb“ renovieren in genau derselben Weise beschrieben wird wie etwa das „Herstellungsverb“ anfertigen, nämlich in Form von (16).

$$
\lambda y \lambda x \lambda e[D O((x, y) r) \& B E C((B E((y) s)) e)]
$$

Da ,etwas herstellen“ und ,etwas bereits Bestehendes modifizieren' jedoch als deutlich unterschiedliche Handlungstypen aufzufassen sind, ist es wenig überzeugend, wenn beide in ein und derselben Weise notiert werden.

Dass die Beschreibungssprache zu stark unterspezifiziert ist, belegt nicht zuletzt die Tatsache, dass die von Winhart (2005: 156) für bringen angenommene Dekomposition in (10') ebenso gut auf zahlreiche weitere Accomplishments anwendbar ist, die in irgendeiner Weise eine zielorientierte Bewegung enthalten, vgl. die Beispiele in (17).

(10') bringen: $\lambda y$ $\lambda x \lambda_{\mathrm{e}}[\mathrm{DO}((\mathrm{x}, \mathrm{y}) \mathrm{r}) \& \operatorname{BEC}((\mathrm{APPL}((\mathrm{y}, \mathrm{a}) \operatorname{APPL}(\mathrm{y}, \mathrm{b}) \mathrm{s})) \mathrm{e})]$

(17) a. Der Handballer wirft den Ball von der Linie aus ins Tor.

b. Ich lege das Buch vom Stuhl auf den Nachttisch. 
Um die möglicherweise entscheidenden semantischen Restriktionen herausarbeiten zu können, die ein Verb den mit ihm kombinierten Nomina auferlegt, ist somit eine detailliertere Beschreibung der Semantik von bringen erforderlich. Diese sollte nicht nur die grammatisch relevanten Struktureigenschaften wie z. B. die Argumentstrukturen und die Ereignisklasse (State, Activity usw.) angeben; sie sollte auch verbspezifische lexikalische Eigenschaften formulieren können. Dazu gehören vor allem auch Angaben zu den semantischen Rollen sowie zu der Art und Weise, in der die Rollen in dem betreffenden Ereignis miteinander interagieren. Die in der Tradition der generativen Semantik stehenden Dekompositionen mittels Prädikaten wie DO, CAUSE, BECOME, von denen auch Winhart Gebrauch gemacht hatte, sind zwar in gewisser Weise als Standard bei der Beschreibung von Verbbedeutungen anzusehen (vgl. u. a. Dowty 1979; Bierwisch 1983; Rappaport Hovav/Levin 1998; Jackendoff 1990; Wunderlich 1996). Sie haben sich aber, wie oben gezeigt, als nicht ausreichend trennscharf erwiesen, wenn es um die Beschreibung von Verbbedeutungen geht.

Aufgrund der nicht ausreichenden Distinktivität der dekompositionellen Beschreibungen mittels DO/CAUSE/BECOME, aber auch wegen ihrer mangelnden psychologischen Plausibilität (Roelofs 1997) sowie vieler weiterer Fragen und Schwierigkeiten hat Engelberg (2000: 17-22; 334-336) eine Repräsentation von Verbbedeutungen in Form von Carnap'schen Bedeutungspostulaten vorgeschlagen (vgl. Carnap 1952: 67). Engelberg (2000: 21) argumentiert, dass für bestimmte semantische Eigenschaften von Verben „Bedeutungspostulate auf jeden Fall benötigt werden“, da diese Eigenschaften nicht durch dekompositionelle Strukturen ausgedrückt werden könnten. So müsse etwa bei der Beschreibung der Bedeutung von öffnen ein Bedeutungspostulat wie in (18b) neben die dekompositionelle Analyse in (18a) treten.
a. öffnen': $\lambda \mathrm{y} \lambda \mathrm{x} \lambda \mathrm{e}[\operatorname{cause}(\mathrm{x}, \mathrm{become}(\mathrm{offen}(\mathrm{y})))]$

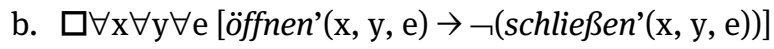

(Engelberg 2000: 21)

Da Bedeutungspostulate im Gegensatz zu den Dekompositionsstrukturen in jedem Fall notwendig seien, hat Engelberg (2000) in der von ihm entwickelten Beschreibungssprache grundsätzlich auf herkömmliche DO/CAUSE/BECOME-Dekompositionen verzichtet und die semantischen Eigenschaften von Verben nahezu ausschließlich mit Hilfe von Bedeutungspostulaten modelliert. Bedeutungspostulate werden auch von Cann (2002) den Dekompositionsstrukturen als das für Lexeme wesentlich geeignetere Beschreibungsinstrument vorgezogen. Eine Stärke von Beschreibungen mittels Bedeutungspostulaten besteht laut Cann (2002) darin, dass gerade die „weicheren“ Eigenschaften von Lexemen besser 
fassbar würden (,Meaning postulates provide a powerful tool for encoding detailed information about the non-logical entailments associated with particular lexemes“, Cann 2002: 552). Wie eine solche Verbbeschreibung auf der Basis von Bedeutungspostulaten aussieht, illustriert Canns Analyse von engl. kiss in (19).

$$
\begin{aligned}
& \forall \mathrm{x} \forall \mathrm{y}[\text { kiss' (x, y) } \leftrightarrow \text { touch' (x, y) \& concrete'(y) \& animate'(x) \& has- } \\
& \text { lips'(x) \& AGEnT(x) \& Theme(y)] }
\end{aligned}
$$

(Cann 2002: 552)

Gleichwohl ist nicht zu bestreiten, dass auch Dekompositionen mittels DO/CAUSE/BECOME Vorteile bei der Beschreibung von Verbbedeutungen bieten. Als linguistisches Beschreibungsinstrumentarium haben sie sich insofern bewährt, als sie auf sehr schlagende Weise Äquivalenzen zwischen Verbbedeutungen verdeutlichen können (vgl. Fanselow/Staudacher 1991: 61f.). Anhand von Dekompositionen lassen sich jedenfalls leicht Generalisierungen über den Verbwortschatz formulieren. Dies belegen die u. a. von Dowty (1979) und Rappaport Hovav/Levin (1998) etablierten Korrespondenzen zwischen Ereignisklassen und Dekompositionsstrukturen, wie sie Tabelle 4 zeigt.

Tab. 4: Entsprechungen zwischen Vendler-Klassen und Dekompositionsstrukturen (Rappaport Hovav/Levin 1998: 108; vgl. auch Dowty 1979: 184; Van Valin 2005: 45)

\begin{tabular}{ll}
\hline$[\mathrm{x}$ act $\langle$ MANNER $\rangle]$ & Activity \\
{$[\mathrm{x}\langle$ STATE $]$} & State \\
{$[\mathrm{BECOME}[\mathrm{x}\langle$ STATE $]]$} & Achievement \\
{$[[\mathrm{x}$ ACT $\langle$ MANNER $]$ CAUSE $[\mathrm{BECOME}[\mathrm{y}\langle$ STATE $\rangle]]$} & Accomplishment \\
& (,indirect causation”) \\
{$[\mathrm{x}$ CAUSE $[\mathrm{BECOME}[\mathrm{y}\langle$ STATE $\rangle]]$} & Accomplishment \\
& (,direct causation”) \\
\hline
\end{tabular}

Da es in diesem Kapitel um die Herausarbeitung von Entsprechungen bzw. Unterschieden zwischen Ereignisversprachlichungen geht, sind Dekompositionsstrukturen mit den Generalisierungen, die sie ermöglichen, somit ein nützliches Instrument. Um den Vorteil von Dekompositionen mittels Do/CAUSE/BECOME etc. zu erhalten und gleichzeitig deren mangelnde Granularität zu kompensieren, sei hier im Hinblick auf die Beschreibungssprache ein Mittelweg gewählt: Als Ausgangspunkt der semantischen Analyse dient eine Dekomposition, diese wird dann in einer zweiten Schicht durch die Angabe von Bedeutungspostulaten er- 
gänzt. Die Dekomposition soll dabei eine grundlegende Klassifikation des jeweiligen Ereignisses nach den Grundtypen in Tabelle 4 leisten. Die zweite Schicht erfasst dann die über diese erste Klassifikation hinausgehenden verbspezifischen Eigenschaften. Da Bedeutungspostulate und Dekompositionen keine einander ausschließenden Beschreibungen darstellen, sondern Dekompositionen grundsätzlich auch in Bedeutungspostulate übersetzt werden können (Chierchia/McConnell-Ginet 2000: 449), seien um der Homogenität der Beschreibung willen hier im Weiteren alle Dekompositionen als Bedeutungspostulate formuliert. Eine solche zweischichtige Analyse gestaltet sich dementsprechend wie in (20).
a. $\forall \mathrm{x} \forall \mathrm{y}\left[\right.$ kiss' $\left.^{\prime}(\mathrm{x}, \mathrm{y}) \rightarrow \mathrm{act}^{\prime}(\mathrm{x}, \mathrm{y})\right]$
b. $\forall x \forall y$ [kiss' ( $x, y) \rightarrow$ touch' $^{\prime}(x, y) \&$ concrete'(y) \& animate'(x) \& has- lips'(x) \& AGENT(x) \& THEME(y)]

Mit dem ersten Teil (20a) dieser Beschreibung ist das Verb kiss allgemein als Activity im Sinne der Muster in Tabelle 4 charakterisiert, und der zweite Teil (20b) gibt die spezifischeren Merkmale an, mit deren Hilfe sich das Verb von anderen Activities abgrenzen lässt.

In der vorliegenden semantischen Beschreibung wird freilich, anders als in Cann's Analyse von engl. kiss in (19), auf eine bikonditionale Relation zwischen Explanandum und Explanans verzichtet, da eine solche Relation für lexikalischsemantische Beschreibungen meist zu stark ist. Cann selbst hatte mit seiner auf Bedeutungspostulaten beruhenden Analyse ja explizit die „non-logical entailments associated with particular lexemes“ (2002: 552) im Blick, und gerade für diese sind exakte Genau-dann-wenn-Beziehungen häufig nicht formulierbar. Dies demonstriert nicht zuletzt Cann's Beispiel kiss, das mit den in (19) angegebenen Bedeutungspostulaten gerade nicht hinreichend beschrieben ist. (Das Postulat „has lips'(x)“ in [19] reicht in jedem Fall nicht für eine adäquate Definition von ,küssen‘.) Für die weiteren Analysen sei hier deshalb ein einfaches Konditional verwendet.

Zweisträngige Analysen, die derjenigen in (20) prinzipiell entsprechen, sind in der lexikalischen Semantik gängig. Meist werden die zwei Stränge von sprachtheoretischen Annahmen über unterschiedliche Ebenen der Semantik her begründet. So unterscheidet Wunderlich (2000) zwischen einer „semantic form“ (SF) und einer „conceptual structure“ (CS). Erstere enthält lediglich diejenigen semantischen Informationen, die für die Syntax relevant sind, während Letztere eine im Prinzip offene Liste von Wissenskomponenten umfasst (vgl. Wunderlich 2000: 250). Noch radikaler verfährt die Distributed Morphology (Halle/Marantz 
1993). Hier wird zwischen „vocabulary entries“ und „encyclopedic entries“ differenziert (Harley/Noyer 2000: 361), wobei die „vocabulary en-tries“ als die eigentlich sprachlichen Komponenten des Lexikons noch stärker unterspezifiziert sind als die vergleichbare SF in Wunderlichs Ansatz.

Für die hier verwendeten zweisträngigen Analysen im Stile von (20) wird keine Korrelation zwischen dem a-bzw. b-Strang und irgendwelchen Ebenen der Bedeutung - etwa einer genuin sprachlichen/syntaktisch relevanten und einer enzyklopädischen Bedeutung - postuliert. Dies ist zunächst damit zu begründen, dass seine solche Differenzierung grundsätzlich problematisch ist, weil die Kriterien für die Unterscheidung zwischen sprachlichen und enzyklopädischen Gegebenheiten nach wie vor unklar sind (vgl. Haiman 1980 sowie die Diskussion in Taylor 1995: 268-294). Gegen eine solche Differenzierung spricht ferner, dass auch Konzepte, die gemeinhin als enzyklopädisch gelten, syntaktisch relevant sein können (vgl. Jackendoff 2002: 290; Engelberg 2011a: 135). Der wichtigere Grund für den Verzicht auf die Zuordnung einzelner Beschreibungskomponenten $\mathrm{zu}$ verschiedenen Bedeutungsebenen ist jedoch schlicht darin $\mathrm{zu}$ sehen, dass eine solche Zuordnung in unserem Zusammenhang keine deskriptiven Vorteile mit sich bringt: Die Frage, ob z. B. das Merkmal „touch’(x,y)“ in (20b) als sprachlich oder enzyklopädisch zu gelten hat, ist für die Beschreibung der lexikalischen Semantik des Verbs unerheblich.

Ein Format wie das in (20) ist sicherlich nur als eine vorläufige Lösung für das Problem einer angemessenen lexikalisch-semantischen Beschreibung von Verben anzusehen. Da die lexikalische Semantik bisher aber insgesamt noch keine breit akzeptierten und auf eine größere Zahl von Fällen anwendbaren Beschreibungswerkzeuge entwickelt hat (vgl. Engelberg 2000: 22; Cruse 2011: 234), ${ }^{91}$ erscheinen solche Zwischenlösungen unausweichlich. Wie eine solche Beschreibung im Einzelnen aussehen kann, sei im Folgenden an der Bedeutung von bringen gezeigt.

91 Cruse (2011: 234) führt zu den bisher entwickelten ,approaches to the description of lexical meaning“ weiter aus: „[A]lthough the majority of them contribute at least some insight to the problem, a satisfying, coherent, comprehensive approach is currently not available“. Ein Grund für diese Situation dürfte darin zu sehen sein, dass viele theoretische Ansätze ihre Konzepte an einer relativ überschaubaren Zahl von Beispielen entwickeln und sich auch auf eine begrenzte Menge von Fragestellungen beschränken. Die „Conceptual Semantics“ von Jackendoff (1990) beschreibt zwar relativ große Teile des Verbwortschatzes (vgl. die Übersicht in Jackendoff 1990: 311-315); die Beschreibungen sind grundsätzlich aber (wie der oben skizzierte Ansatz von Wunderlich 2000 auch) auf die Syntax-Semantik-Schnittstelle ausgerichtet und lassen häufig die für lexikalische Fragestellungen erforderliche Granularität vermissen; vgl. die Kritik von Geeraerts (2010: 140f.). 


\subsubsection{Die Bedeutung von bringen}

\subsubsection{Lesarten}

Für die Beschreibung von bringen in FVG ist zunächst festzuhalten, dass kaum alle Bedeutungen aus dem reichhaltigen semantischen Spektrum des Verbs hier überhaupt einschlägig sind. Verwendungen des Verbs wie in Die Zeitung bringt einen schönen Artikel, Mein Konto bringt kaum Zinsen oder Der Manager bringt die Leistung nicht sind offenbar metaphorisch und bilden sicher nicht die Basis für das FV bringen. Es kommt hier vielmehr nur die Verblesart in Betracht, von welcher am ehesten alle weiteren Bedeutungen im semantischen Spektrum von bringen motiviert sind, nämlich das Bewegungsverb, wie es in den Beispielen in (21) vorliegt.
(21) a. Das Mädchen bringt das Buch zur Bibliothek/in die Bibliothek.
b. Der Mann bringt den Gast zum Bahnhof.
c. Die Bibliothekarin brachte der Studentin das Buch.

Die Beispiele in (21) bezeichnen zwar gleichermaßen ein konkretes Ereignis im Raum, sie sind freilich nicht vollkommen identisch: (21a) versprachlicht einen Transport, (21b) kann ohne weiteren Kontext sowohl als Transport als auch als Begleitung interpretiert werden, und (21c) hebt sich insofern von den beiden anderen Beispielsätzen ab, als das Verb hier nicht mit einer PP, sondern mit einem Dativobjekt konstruiert ist. Bringen zeigt also die sog. Dativalternation (vgl. Pinker 1989: 110f.; Adler 2011). Bringen mit Dativobjekt stellt auch in semantischer Hinsicht eine Besonderheit dar, weil hier nicht (nur) eine Bewegung, sondern auch ein Besitzwechsel kodiert wird: In (21c) verfügt Susi nach Abschluss des bringen-Ereignisses über den Gegenstand ,Buch‘. Für bringen werden daher mit Recht zwei grundlegende Lesarten angesetzt: Bringen mit PP bezeichnet eine ,caused motion', bringen mit Dativobjekt eine ,caused possession“ (Adler 2011: 216; zu der Unterscheidung vgl. auch Pinker 1989: 110f.; Levin 1993: 46, 114). Für bringen in FVG kann die ,caused possession'-Lesart vernachlässigt werden, und zwar schlicht deshalb, weil das Verb in FVG nur mit PP und nie mit Dativobjekt auftritt. Daher ist bringen im Zusammenhang dieser Arbeit als ein Fall von ,caused motion' zu beschreiben. ${ }^{92}$

92 Ob bringen, wenn es keinen Transport, sondern eine Begleitung wie in Hans brachte den Gast zur Tür kodiert, ebenfalls unter ,caused motion' zu behandeln wäre, sei dahin gestellt. Wenn überhaupt, so handelt es sich hier um eine sehr spezielle Ausprägung von ,caused motion‘, die 


\subsubsection{Bringen als, caused-motion'-Verb}

,Caused motion' liegt vor, wenn ein Ereignis grundsätzlich anhand der Struktur in (22) beschrieben werden kann.

(22) $x$ causes $y$ to be at $z^{93}$

(vgl. Levin 2008: 285)

Eine solche Struktur trifft allerdings auf eine relativ große Zahl von Verben zu, sofern diese mit Akkusativobjekt und der Angabe eines Zieles versehen sind, vgl. die Fälle in (23).

(23) a. den Ball ins Spielfeld werfen

b. den Wagen in die Garage rollen

c. das Wasser in den Pool spritzen

Mit einer Charakterisierung von bringen als Fall von caused motion ist somit lediglich eine erste Einordnung erreicht. Entscheidendes Merkmal der Bedeutung von bringen, durch das es sich von anderen caused-motion-Ereignissen abgrenzen lässt, ist die Art und Weise, in der das Bewegungsereignis kontrolliert wird. Bei werfen in (22a) wird das Erreichen des Ziels dadurch gewährleistet, dass eine Steuerung des Werfens am Beginn des Ereignisses steht: Der Ball wird so geworfen, dass er einen bestimmten Pfad in Richtung auf das Ziel nimmt. Die Kontrolle des Ereignisses durch den Agens gilt hier also nur für die Initialphase von werfen. Bei bringen hingegen ist die Kontrolle des Ereignisses nicht auf den Beginn der Bewegung begrenzt, sondern erstreckt sich zeitlich über das gesamte Ereignis und räumlich über den gesamten Pfad des Patiens. Der Agens hat hier permanent sicherzustellen, dass der Patiens sich bewegt und dass dieser sich genau auf dem Pfad bewegt, der der Intention des Agens entspricht. Das übliche Mittel dieser konstanten Ereigniskontrolle ist die Begleitung des Patiens durch den Agens auf seinem Pfad. Die Tätigkeit des Agens von bringen kann daher mit Ehrich (1996: 244f.) als ,Antrieb“ charakterisiert und vom ,Anstoß` etwa bei werfen abgegrenzt

eng mit einem kulturellen Konzept von Höflichkeit verknüpft ist. Daran, dass bringen in FVG als ,caused motion'-Ereignis zu beschreiben ist, ändert auch dieser Sonderfall nichts.

93 Ehrich (1996: 235) beschreibt ,caused motion“ auch als „Transport“: „Ein Transport ist ein Vorgang, bei dem ein AGENS ( $\mathrm{x}$ ) auf ein PATIENS (y) einwirkt, so dass y ausgehend von seinem URSPRUNGSort (u) längs einen WEGES (w) seinen Ort kontinuierlich ändert und schließlich zu dem ZIELort (z) gelangt“. 
werden (vgl. auch die Unterscheidung von zwei Arten des Bewirkens von Bewegungsereignissen - entraining und launching - bei Jackendoff 1990: 138).

Von anderen caused-motion-Verben mit ,Antrieb“ wie z. B. setzen, stellen, legen ist bringen durch das Vorhandensein eines Pfades abzugrenzen. Das Bewegungsereignis, das die eben genannten Verben versprachlichen, hat zwar ein Ziel, es liegt jedoch kein signifikanter Pfad vor, wie (24a) zeigt. Ein Satz mit bringen kann dagegen ein explizites Pfad-Argument enthalten, vgl. (24b). Auch wenn das Pfad-Argument nicht obligatorisch ist, setzt bringen dennoch das Vorhandensein eines Pfades voraus. Dies belegt die ungrammatische Äußerung (24c), die deshalb nicht akzeptabel ist, weil hier eine zu große räumliche Nähe zwischen Patiens und Ziel vorliegt und folglich kein Pfad existieren kann.

(24) a. `Er stellte die Blumen über den Flur in die Vase.

b. Er brachte den Gast über die Wilhelmstraße zum Bahnhof.

c. ${ }^{\star}$ Er brachte die Blumen in die Vase.

Das Gegebensein eines Pfades kann übrigens auch als Bedingung für die permanente Agenspartizipation, d. h. den ,Antrieb،, gesehen werden: Ein Pfad, d. h. eine signifikante Entfernung zwischen Ausgangspunkt und Ziel, erfordert die Bewegung des Agens als Ganzen; die Bewegung bloß eines Teils (typischerweise des Armes) ist zwar bei setzen, stellen, legen für eine Kontrolle des Pfades ausreichend, bei bringen jedoch nicht. Dies erklärt auch, weshalb Sätze wie (25b) gegenüber (25a) weniger akzeptabel erscheinen.

(25) a. Sie brachte das Buch in die Bibliothek.

b. 'Sie brachte das Buch ins Bücherregal.

Bringen setzt somit voraus, dass der Agens als Ganzer auch am Endpunkt der Bewegung anwesend ist. Das ist bei ins Bücherregal in (25b) nicht möglich - der Agens findet als Ganzer kaum Platz im Bücherregal -, weshalb der Satz fragwürdig klingt. Stellen und legen sind hier als Alternativen unproblematisch.

Die Beschreibung von bringen als caused-motion-Ereignis in (26a) ist - so kann hier gefolgert werden - zunächst um die zwei Postulate in (26b) zu ergänzen.

(26) a. bringen: $\forall \mathrm{x} \forall \mathrm{y} \forall \mathrm{z}[\operatorname{BringEN}(\mathrm{x}, \mathrm{y}, \mathrm{z}) \rightarrow \operatorname{CAUSE}(\mathrm{x}, \operatorname{BECOME}(\operatorname{AT} \operatorname{GOAL}(\mathrm{y}, \mathrm{z})))]$

b. $\forall \mathrm{x} \forall \mathrm{y} \forall \mathrm{e}\left[\operatorname{BRINGEn}(\mathrm{x}, \mathrm{y}, \mathrm{e}) \rightarrow \exists \mathrm{p}(\operatorname{PATH}(\mathrm{p}, \mathrm{e})) \& \exists \mathrm{e}^{\prime}\left(\operatorname{CONTROL}_{\text {perm }}(\mathrm{x}, \mathrm{e}, \mathrm{e})\right.\right.$ by $\operatorname{PART}(\mathrm{x}, \mathrm{e})))$ 
Das erste Postulat in (26b) bezieht sich auf das Gegebensein eines Pfades, das zweite auf die Kontrolle des Patiens durch den Agens. Durch das Subskript „perm“ wird ausgedrückt, dass der Agens den Patiens nicht nur am Beginn des Ereignisses (Initialkontrolle, ,Anstoß`), sondern während des gesamten Ereignisses kontrolliert. „by PART (x, e)“ schließlich ist eine Angabe der Art und Weise, in der die permanente Ereigniskontrolle ausgeübt wird, nämlich durch Partizipation des Agens an der Bewegung des Patiens.

Ein weiterer Aspekt der Bedeutung von bringen, der für das Verständnis seiner Kombinationen besonders wichtig ist, betrifft eine Eigenschaft, die es mit vielen caused-motion-Prädikaten teilt und die daher nicht sogleich in den Blick kommt. So ist für die meisten Bewegungsereignisse charakteristisch, dass sich der Patiens im Zuge der Bewegung nicht ändert: Wenn jemand den Kaffee an den Tisch bringt, dann ist der Patiens am Ziel mit dem Patiens am Ausgangspunkt identifizierbar, auch wenn er in akzidentellen Eigenschaften verändert sein kann - der Kaffee mag nach Abschluss der Transports etwas kälter werden, er ist aber unmittelbar als Kaffee erkennbar. Winhart (2005: 156) hatte diese Eigenschaft zutreffend als referenzielle Identifizierbarkeit des Objekts im Endzustand mit dem Objekt im Vorzustand beschrieben (s. o. Abschnitt 5.2.1). Diese Identifizierbarkeit liegt freilich nicht bei allen caused-motion-Prädikaten vor, vgl. die Beispiele in (27).

(27) a. Hans streute die Brocken auf den Boden.

b. Greta krümelt das Brot in die Suppe.

Hier ist zwar ebenfalls die Grundstruktur „, $\mathrm{x}$ causes y to be at z“ gegeben, der Patiens ändert aber sehr wohl seine innere Beschaffenheit im Zuge des Ereignisses. Für bringen ist die Unveränderlichkeit der Patienskonstitution freilich wesentlich. Daher klingt eine Äußerung wie (28b) gegenüber (28a) merkwürdig.

(28) a. Der Geldtransporter hat die Tageseinnahmen zur Bank gebracht. Es fehlen jedoch kleinere Beträge.

b. 'Der Geldtransporter hat die Tageseinnahmen zur Bank gebracht. Es fehlt jedoch 90\% des Geldes.

Die Beschreibung der Bedeutung von bringen in (26) ist somit um ein weiteres Postulat zu ergänzen, das die Unveränderlichkeit der Patienskonstitution festschreibt. Daraus ergibt sich die Notation in (29). 

a. bringen: $\forall \mathrm{x} \forall \mathrm{y} \forall \mathrm{z}[\operatorname{BRINGEN}(\mathrm{x}, \mathrm{y}, \mathrm{z}) \rightarrow \operatorname{CAUSE}(\mathrm{x}, \operatorname{BECOME}(\operatorname{AT~GOAL}(\mathrm{y}, \mathrm{z}))$ )]
b. $\forall \mathrm{x} \forall \mathrm{y} \forall \mathrm{e}$ [BRingen( $\mathrm{x}, \mathrm{y}, \mathrm{e}) \rightarrow \exists \mathrm{p}(\operatorname{PATH}(\mathrm{p}, \mathrm{e}))$ \& $\exists$ e'(CONTROL $(\mathrm{x}, \mathrm{e}, \mathrm{e})$ by PART (x, e)) \& $\neg \exists$ e’(CHANGE OF STATE $(x, y, e ”))]$

\subsubsection{Bringen und die Beschränkungen in der Kombinierbarkeit}

\subsubsection{Semantische Vereinbarkeit zwischen Verb und Nomen}

Wenn man der Arbeitshypothese von Winhart (2005) folgend davon ausgeht, dass zwischen der Bedeutung des Verbs bringen und den Nomina in FVG eine wie auch immer geartete semantische Übereinstimmung besteht, so stellt sich die Frage, ob diese Hypothese nicht sehr einfach falsifiziert werden kann: Bringen ist ein Bewegungsverb, während die Nomina in bringen-FVG gerade keine Bewegungsereignisse bezeichnen. Die Suche nach semantischen Äquivalenzen zwischen Verben unterschiedlicher semantischer Domänen wäre demnach von vornherein zum Scheitern verurteilt. Soll diese Arbeitshypothese weiter verfolgt werden, ist sie daher abzuschwächen, und zwar insofern, als es nur um wortfeldübergreifende Strukturentsprechungen zwischen dem Bewegungsereignis bringen und den in den Nominalisierungen ausgedrückten Ereignissen gehen kann.

Dass es zwischen Bewegungsverben und Verben anderer Wortfelder zahlreiche strukturelle Parallelen gibt, ist bereits mehrfach festgestellt worden. So werden in Jackendoffs „Conceptual Semantics“ (Jackendoff 1983; 1990) Bewegungskonzepte als grundlegend für die Konzeptualisierung von Ereignissen aufgefasst. Daraus folgt für die Beschreibung von Verben: „[T]he formalism for encoding concepts of spatial location and motion, suitably abstracted, can be generalized to many other semantic fields“ (Jackendoff 1990: 25; vgl. 1983: 188). Jackendoff sieht dabei freilich die räumlichen Konzepte selbst als Instanzen eines abstrakten Schemas, das sich in verschiedenen Domänen (,semantic fields“) manifestieren kann. Es handelt sich jeweils um „parallel instantiations of a more abstract schema“ (Jackendoff 2002: 359). So ist Jackendoff zufolge das Primitiv Go gleichermaßen in den Feldern „Location“, „Possession“ und „Predication“ vertreten:

(30) a. The messenger went from Paris to Istanbul. (Location)

b. The inheritance finally went to Fred. (Possession)

c. The light went from green to red. (Predication)

(vgl. Jackendoff 1990: 25f.; 2002: 356)

In der kognitiven Linguistik wird die Parallelität zwischen Bewegungs- und Ereigniskonzepten als Resultat einer metaphorischen Übertragung beschrieben, 
die dem Muster CHANGE OF STATE IS CHANGE OF LOCATION bzW. CHANGE IS MOTION folgt (Lakoff/Turner 1989: 8; Radden 1996: 425). Im Zuge der Übertragung von der Domäne der Bewegung bzw. des Ortswechsels auf nicht-lokale Ereignisse werden dabei, so die Annahme, die strukturellen Elemente des Bewegungsereignisses auf das nicht-lokale Ereignis übertragen. Der Ausgangspunkt der Bewegung ist dann mit dem Vorzustand, der Pfad mit der Übergangsphase und das Ziel mit dem Resultatszustand zu identifizieren (vgl. die sog. „Invariance Hypothesis“ von Lakoff 1990: 54).

Wie auch immer man die Übereinstimmungen zwischen Bewegungsverben und Verben anderer Wortfelder modelliert, es erscheint vor dem Hintergrund dieser Überlegungen jedenfalls alles andere als aussichtslos, nach Parallelen zwischen bringen und den Verben $\mathrm{zu}$ fragen, die die N-Position in entsprechenden FVG besetzen. Dabei hat man - in diesem Punkt besteht Einigkeit zwischen Jackendoff und den Vertretern der kognitiven Linguistik - von wortfeldspezifischen semantischen Merkmalen abzusehen und stattdessen strukturelle Entsprechungen herauszuarbeiten, die auf einem relativ hohen Abstraktionsniveau liegen. Dies können sein:

- Übereinstimmungen hinsichtlich Transitivität/Intransitivität des Verbs,

- Entsprechungen zwischen den jeweils vorliegenden Ereignistypen (statisches/dynamisches Ereignis bzw. genauer die Unterscheidung zwischen State, Activity, Accomplishment und Achievement),

- Entsprechungen bei den semantischen Rollen und ihrer Interaktion (permanente Kontrolle/Initialkontrolle usw.) .

Was die ersten beiden Punkte angeht, ergeben sich freilich keine besonders signifikanten Übereinstimmungen zwischen bringen und den Basisverben der einschlägigen Nomina, die hier nochmals in (31) wiedergegeben seien.

(31) Abbruch, Abschluss, Anklage, Anzeige, Anwendung, Aufführung, Ausführung, Darstellung, Darbietung, Druck, Durchführung, Einsatz, Umsetzung, Verbreitung, Verkauf, Verlesung, Veröffentlichung, Verteilung, Versteigerung, Vollendung, Vortrag

Selten sind (mit weniger als fünf Treffern in DeReKo [Stand 15.03.2013] bzw. anderer Quelle entnommen): Aufruf (Kontext Auktion), Aussaat, Ausstrahlung, Fertigstellung, Meldung, Sendung, Verrechnung)

Alle Basisverben sind hier (wie bringen) transitiv bzw. haben eine transitive Lesart; angesichts der Vielzahl der existierenden transitiven Verben ergibt sich daraus, wie angedeutet, keine weiterführende Differenzierung. Die Beschränkungen 
in der Besetzung der N-Position werden erst dann verständlich, wenn man bei den einzelnen Positionen der Bedeutungsbeschreibung in (29) ansetzt.

\section{a) Ereignistyp}

Im Hinblick auf den Ereignistyp der Nominalisierungen in bringen-FVG kann festgehalten werden, dass es sich auch bei den allermeisten Basisverben um Accomplishments handelt. Dies lässt sich grundsätzlich durch die Kombinierbarkeit des transitiven Verbs mit einem Zeitrahmenadverbial in/innerhalb belegen (zu diesem gängigen Test vgl. Lohnstein 1996: 232f.). Die entsprechenden Testsätze in (32) weisen neben einer Reihe sehr guter Beispiele indes auch einige etwas schwierigere Fälle auf.

(32) etwas innerhalb von drei Minuten abbrechen etwas innerhalb von drei Minuten abschließen das Gesetz innerhalb weniger Tage anwenden ein Verbrechen innerhalb kürzester Zeit anzeigen die Oper in drei Stunden aufführen Der Computer führte das Programm innerhalb weniger Sekunden aus komplexe Gedanken in wenigen Minuten darstellen Der Drucker kann einen langen Text in wenigen Minuten drucken die Suppe innerhalb von zehn Minuten verteilen eine Maßnahme innerhalb kürzester Zeit durchführen die Wirkung entfaltet sich innerhalb von wenigen Minuten etwas innerhalb von zwei Stunden entscheiden die Bilder waren in zwei Minuten versteigert seine Gedanken innerhalb von fünf Minuten vortragen

Was die ersten beiden Punkte angeht, ergeben sich freilich keine besonders signifikanten Übereinstimmungen zwischen bringen und den Basisverben der einschlägigen Nomina, die hier nochmals in (31) wiedergegeben seien.

(33) a. Er brachte den Gast in/innerhalb von 20 Minuten zum Bahnhof.

b. `Er brachte den Gast 20 Minuten lang zum Bahnhof.

Einige Verben entziehen sich freilich einer genauen Einordnung hinsichtlich ihres Ereignistyps. Dies gilt besonders für einsetzen (in zum Einsatz bringen). Hier ist keine Kombination mit einem Zeitrahmenadverbial möglich: 
(34) a. Der Trainer setzte den Spieler nur drei Minute lang ein.

b. \#Der Trainer setzte den Spieler innerhalb von drei Minuten ein.

Gleichwohl verhält sich einsetzen im Hinblick auf seine semantische Struktur in gewisser Weise wie ein Accomplishment, da es ebenfalls nach dem Muster [x CAUSE (BECOME $(\mathrm{y}<S T A T E>))]$ beschrieben werden kann, zumindest wenn man als STATE hier so etwas wie ,im Spiel sein“ oder ,in Gebrauch sein“ voraussetzt. Der Trainer setzt den Spieler ein wäre dann zu paraphrasieren als ,Der Trainer bewirkt, dass der Spieler im Spiel ist“, Der Polizist setzt eine Waffe ein als ,Der Polizist bewirkt, dass eine Waffe im Gebrauch ist‘.

Mit der Zuordnung der einschlägigen Basisverben zum Ereignistyp Accomplishment, die zumindest für die allermeisten Fälle in (32) plausibel gemacht werden kann, ist der Bestand der Kandidaten für die Besetzung der N-Position in bringen-FVG etwas überschaubarer geworden: States, Activities und Achievements sind keine Quellen für ein entsprechendes Nominal. ${ }^{94}$ Blickt man auf semantische Verbklassen etwa in der Art der von Ehrich/Rapp (2000: 279-281) definierten Gruppen, kann damit gleich eine ganze Reihe von Nominalisierungen ausgeschlossen werden:

(35) States:

a. psychische Einstellungsverben: ${ }^{\star}$ zur Verehrung bringen, ${ }^{\star}$ zur Verachtung bringen, ${ }^{\star}$ zur Bewunderung bringen

b. Besitzverben: ${ }^{\star}$ zum Besitz bringen

Activities:

a. Behandlungs-/Betreuungsverben (Activities): \#zur Behandlung bringen, \#zur Pflege bringen, \#zur Unterstützung bringen, \#zur Beratung bringen

b. Kampfverben: \#zur Bekämpfung bringen, \#zur Belagerung bringen, \#zur Verfolgung bringen

c. Investigationsverben: \#zur Besichtigung bringen, \#zur Befragung bringen, \#zur Untersuchung bringen, \#zur Messung bringen

94 Es gibt zwar einzelne Verbindungen, die sehr wohl bringen $+z u$ mit einer State- bzw. ActivityNominalisierung verbinden, etwa zur Ruhe, zum Stehen, zum Sprechen, zum Sieden, zum Keimen bringen. Dies sind aber keine FVG im Sinne dieser Untersuchung, vgl. dazu Kapitel 2.3.1. 
Achievements:

Nichtkausative Zustandsveränderungsprädikate (Ehrich/Rapp 2000: 281): *zur Verdunstung bringen, *zur Erreichung bringen

Einige der Activities in (35), z. B. befragen, behandeln, untersuchen, weisen zwar auch eine Accomplishment-Lesart auf. Diese ist mit Ehrich/Rapp (2000: 279, Fußnote 33) aber als sekundär gegenüber der Activity-Interpretation anzusehen. Die Verwendungskontexte, in denen ein Rahmenadverbial in/innerhalb möglich ist und die daher eine Accomplishment-Interpretation nahelegen, sind hier in der Tat relativ spezifisch, vgl. dazu das Beispiel in (36b), das gegenüber dem unmarkierten (36a) nur in besonderen Kontexten sinnvoll ist, d. h. hier bei einem Vergleich der jeweiligen Handlungsdauer.

(36) a. Der Oberarzt untersucht den Patienten eine Stunde lang.

b. Der Oberarzt untersucht den Patienten in einer Stunde, der Chefarzt schafft es in zwei Stunden.

\section{b) Permanente Ereigniskontrolle}

Mit der Einschränkung auf Accomplishments ist zwar eine wichtige Eigenschaft der Nominale in FVG mit bringen (zu) benannt. Dies ist freilich noch nicht ausreichend, da durchaus nicht jedes Accomplishment in Frage kommt. Für die Herausarbeitung weiterer Selektionsbeschränkungen erweisen sich besonders die Eigenschaften von bringen als relevant, die im b-Teil der Beschreibung in (29) enthalten sind.

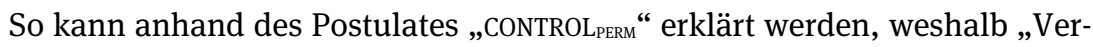
fügbarkeitsverben“ (Ehrich/Rapp 2000: 283) wie entdecken, ausgraben oder erfinden nicht im Nominal eines bringen-FVG vorkommen, vgl. (37).

*einen Kontinent zur Entdeckung bringen, *eine Statue zur Ausgrabung bringen, *eine Stadt zur Eroberung bringen

Im Fall von entdecken und ausgraben findet keine über die gesamte Handlung sich erstreckende Einwirkung des Agens auf den Patiens statt, da der Patiens erst nach Abschluss der Handlung für den Agens überhaupt verfügbar ist. Die für bringen wesentliche Komponente der permanenten Kontrolle des Patiens durch den Agens ist hier somit nicht gegeben.

Das von Winhart (2005: 161) herausgearbeitete Kriterium der Vorexistenz des Patiens (bzw. der konstanten Referierbarkeit), das die Nicht-Akzeptabilität von 
Herstellungs- und Vernichtungsverben durchaus erklären kann (s. o. Abschnitt 5.2.1), bietet für diese Verbgruppe übrigens keine Lösung. Bei erobern, entdecken und ausgraben ist der Patiens sehr wohl auch vor Erreichen des Endzustandes existent, er steht eben nur nicht unter der Kontrolle des Agens. Der Bedeutungs-

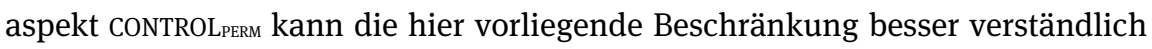
machen.

Die für bringen kennzeichnende Kontrolle des Patiens durch den Agens ist möglicherweise auch für eine Auffälligkeit bei den Verben des Besitzwechsels verantwortlich. Verben wie kaufen, erwerben, bestellen, anfordern sind, obwohl sie tendenziell Accomplishment-Charakter besitzen, nicht in bringen-FVG als Basen belegbar, vgl. (38).
a. *einen Wagen zum Kauf bringen
b. *einen Mantel zur Bestellung bringen

Für die Verben des Besitzerwerbs gilt nun gerade, dass der Constraint ,Agens kontrolliert Patiens permanent' nicht gegeben ist. Wenn jemand etwas kauft, dann kontrolliert er den Patiens erst nach Vollzug der Handlung; während des Kaufs besitzt der Agens jedoch keine Kontrolle über das zu Kaufende (der Verkäufer kann dem Kauf z. B. nicht zustimmen, der Kaufgegenstand kann auf dem Weg zum Käufer verloren gehen usw.).

Mit der andauernden Patiens-Kontrolle als Merkmal des Verbs bringen hängt wohl auch zusammen, dass verkaufen im Gegensatz zu kaufen durchaus in einem entsprechenden FVG vorkommen kann:

(39) a. Ziel des OGV ist es, selbst produzierte Erzeugnisse zum Verkauf zu bringen. (Frankfurter Rundschau, 23.09.1999, S. 9; Zugriff 06.04.2021).

b. Schieles beschlagnahmtes Bildnis „Tote Stadt III“ ist offenbar von der Schwägerin von Fritz Grünbaum 1956 beim Berner Kunsthaus Klipstein \& Kornfeld zum Verkauf gebracht worden (Vorarlberger Nachrichten, 16.01.1998, S. D6; Zugriff 06.04.2021).

Im Fall von verkaufen/Verkauf kontrolliert der Agens den Patiens solange, bis das Verkaufsereignis vollzogen ist. Bei kaufen kommt die Kontrolle des Agens erst nach Abschluss der Transfers zustande. Es ist folglich semantisch nicht mit bringen kompatibel. 


\section{c) Konstanz der Patienskonstitution}

Eine weitere für die Selektion der Nomina relevante Eigenschaft von bringen ist die Unveränderlichkeit der Patienskonstitution im Zuge des Ereignisses $(\neg \exists \mathrm{e}$ ” CHANGE OF STATE (x, y, e”)). Diese kann den Ausschluss zweier großen Klassen von Accomplishments erklären: der großen Gruppe der „Modifikationsverben“ (Ehrich/Rapp 2000: 284) sowie der Ornativa. Zu den Modifikationsverben gehören etwa die Fälle in (40).

(40) umarbeiten, kürzen, modifizieren, renovieren, umgestalten, verändern

Diese Verben zeichnen sich dadurch aus, dass der Patiens nach Abschluss des Ereignisses einen Nachzustand erreicht, der sich von dem jeweiligen Vorzustand erkennbar unterscheidet. Änderungen der Konstitution eines Objekts sind prinzipiell nach ihrem Grad unterscheidbar - etwas kann stark oder nur ein wenig verändert werden. Daher eignen sich Gradadverbiale wie leicht, stark oder gründlich als Testrahmen für Modifikationsverben.

(41) a. Der Text wird stark/leicht gekürzt.

b. Das Haus wird gründlich/oberflächlich renoviert.

Verben wie kürzen unterscheiden sich damit in ihrer semantischen Struktur deutlich von bringen, das nicht mit Gradadverbial konstruierbar ist. Die Nicht-Akzeptabilität von Modifikationsverben bzw. darauf beruhender Nominalisierungen in FVG, die durch Äußerungen wie in (42) illustriert wird, erscheint somit naheliegend.

(42) a. *Sie brachte den Text zur Kürzung.

b. ${ }^{\star}$ Er brachte das Haus zur Renovierung.

Eigens hinzuweisen ist in diesem Zusammenhang allerdings nochmals auf das Verhalten von Verben wie vollenden und abschließen. Bereits aus den oben referierten Überlegungen von Winhart (2005) zum Kriterium der Vorexistenz des Objekts war zu schließen, dass es sich bei beiden Verben nicht um Modifikationsverben im eigentlichen Sinne handelt: Mit Vollendung und Abschluss wird der Patiens nicht in der Weise verändert, dass von einem neuartigen Zustand die 
Rede sein könnte. ${ }^{95}$ Dies wird auch durch das negative Ergebnis der einschlägigen Tests bestätigt:

a. `Er hat den Dom intensiv/gründlich/stark vollendet.

b. *Die Seminararbeit wurde intensiv/gründlich/stark abgeschlossen. ${ }^{96}$

Dass Ornativa (bemalen, verschmutzen, einölen, buttern) grundsätzlich nicht in FVG mit bringen auftreten, vgl. (44), kann ebenfalls anhand des Postulats „ $\neg$ e”CHANGE OF STATE (x, y, e’) erklärt werden.

\section{(44) a. ${ }^{\star}$ Die Kinder brachten den Teppich zur Beschmutzung.}

b. ^Der Künstler brachte das Bild zur Bemalung.

Wenn der Teppich beschmutzt wird, geht er von einem Zustand UNBESCHMUTZT in einen Zustand BESCHMUTZT über. ${ }^{97}$ Das Vorliegen eines Zustandswechsels bei Ornativa kann auch dadurch belegt werden, dass diese mit Prädikaten verbindbar sind, die eine vorhandene oder ausbleibende Veränderung zwischen Ausgangsund Endzustand zum Thema machen.

95 Vollenden wird bei Ehrich/Rapp (2000: 284) allerdings zu den Herstellungsverben gerechnet. Die oben angeführten Argumente sprechen allerdings eher gegen eine solche Einordnung.

96 Die mit vollenden und abschließen in gewisser Weise vergleichbaren Verben abbrechen, stoppen und beendigen sind grundsätzlich als punktuell zu bewerten. Sie sind jedenfalls eher im Zeitpunkt- als mit Zeitrahmenangaben verbindbar.

97 Brinkmann (1995: 53) bezweifelt, dass die Veränderung eines Zustands ein notwendiges Merkmal von Applikativa ist. Brinkmann illustriert ihre These u. a. an dem Satz Donna bestreut den Kuchen mit Zucker: „[T]he verb does not specify that the cake necessarily undergoes any particular change of state, e. g., the sugar may end up on the cake in a thin or thick layer, or even in little heaps. As long as the sugar moves in a distributed manner, the action may be reffered to as bestreuen"(ebd.). Dennoch ist festzuhalten, dass der Resultatszustand von bestreuen eine Situation ist, in der eine ausreichend große Fläche des Kuchens mit Zucker bedeckt ist. Ob der Kuchen dabei nur leicht bestäubt oder mit einer dicken Schicht Zucker überdeckt ist, ist eine Frage des Grades. Dass Resultatszustände unterschiedliche Grade aufweisen, ändert nichts an der Tatsache, dass ein neuer Zustand des Kuchens erreicht ist, auf den referiert werden kann. Auch Brinkmanns Beispielsatz Ununterbrochen berieselt das Kind die Rutsche mit Sand (ebd.) kann ihre These, be-Verben involvierten keine Zustandsveränderung des Objekts, nicht belegen; dazu ist das Beispiel mit dem sehr spezifisch geformten Objekt Rutsche zu ,gesucht'. Außerdem scheint die Akzeptabilität dieses Satzes fragwürdig. Ununterbrochen lässt das Kind Sand auf die Rutsche rieseln ist besser zu bewerten. 
(45) a. Der Teppich sieht jetzt beschmutzt aus.

b. Der Teppich bleibt beschmutzt.

Teilweise sind auch bei den Ornativa adverbiale Angaben des Grades möglich, vgl. (46). Diese weisen ebenfalls darauf hin, dass hier ein meßbarer Zustandswechsel des Patiens vorliegt.

(46) a. Der Teppich ist leicht/stark beschmutzt.

b. Die Wand ist nur leicht/über und über mit Graffiti besprüht.

Dass auch Herstellungs- und Vernichtungsverben (Ehrich/Rapp 2000: 284) mit den Bedeutungsaspekt ,keine Änderung der Konstitution' unvereinbar sind, ist leicht einzusehen, da der Patiens von bringen im Zuge des Ereignisses weder hergestellt noch vernichtet wird. (Dies hatte zumindest für die Herstellungsverben bereits Winhart [2005: 156] mit dem von ihr formulierten Kriterium der referenziellen Identifizierbarkeit richtig vorhergesagt.) In einem FVG mit bringen $(z u)$ treten folglich keine Verben auf, die eine Herstellung oder Vernichtung ihres Objekts bezeichnen, vgl. (47):

(47) a. *die Hausarbeit zur Abfassung bringen

b. *die Stadt zur Zerstörung bringen

\subsubsection{Semantische Eigenschaften der Nomina}

Da hier bisher überwiegend die Verbklassen behandelt wurden, die nicht in bringen-FVG auftreten konnten, ist gewissermaßen ex negativo deutlich geworden, in welcher Weise die Bedeutung von bringen den Nominalen Beschränkungen auferlegt. Im Folgenden ist nun noch einmal kurz der Gegentest zu machen und zu prüfen, ob die Nomina, die in einem FVG mit bringen auftreten, den postulierten semantischen Eigenschaften von bringen auch tatsächlich entsprechen. Es handelt sich um die Nomina in (48).

(48) Abschluss, Anklage, Anzeige, Anwendung, Aufführung, Ausführung, Darstellung, Darbietung, Druck, Durchführung, Einsatz, Umsetzung, Verbreitung, Verkauf, Verlesung, Veröffentlichung, Verteilung, Versteigerung, Vollendung, Vortrag 
selten sind (mit weniger als fünf Treffern in DeReKo bzw. anderer Quelle entnommen): Aufruf (Kontext Auktion), Aussaat, Ausstrahlung, Fertigstellung, Meldung, Sendung, Verrechnung)

Für die Verträglichkeit mit bringen hat sich neben dem Accomplishment-Charakter vor allem die Art und Weise als entscheidend erwiesen, in der Agens und Patiens eines Verbs bzw. seiner Nominalisierung jeweils miteinander interagieren: (a) der Agens kontrolliert den Patiens während des gesamten Ereignisverlaufs und (b) der Patiens unterliegt keiner Veränderung seiner Konstitution.

Das Kriterium (a) ,Agens kontrolliert Patiens‘ ist ohne Zweifel für alle Nominalisierungen in (48) einschlägig: Wenn jemand ein Stück aufführt, ein Bild versteigert usw., handelt es sich stets um Ereignisse, die ohne die Initiative des Agens nicht zustande kämen und die durch Handlungen des Agens (und gegebenenfalls durch dessen mehrfache Intervention) auf das von ihm gewünschte Ziel hin gesteuert werden (vgl. die von Brennenstuhl 1982: 89f. eingeführten Kriterien für ,Kontrolle‘).

Kriterium (b) trifft auf die allermeisten Nomina zu: Ein Theaterstück bleibt es selbst, auch wenn es aufgeführt wird, und es geht nicht in einen Zustand über, der nach einer Aufführung ein erkennbar anderer wäre. Gleiches gilt für ein $\mathrm{Mu}$ sikstück, das vorgetragen wird, eine Dissertation, die gedruckt wird, ein Bild, das versteigert wird, ein Gesetz, das angewandt wird, eine Idee, die dargestellt wird usw. Auch bei Verben wie vollenden und abschließen liegt, wie oben dargelegt, keine Zustandsveränderung des Patiens vor, zumindest nicht im Hinblick auf essentielle Objekteigenschaften.

Lediglich bei dem eher seltenen zur Verteilung bringen stellt sich die Frage, ob hier nicht doch eine Zustandsveränderung enthalten ist: Wenn ein Kuchen verteilt ist, kann er kaum mehr als derselbe angesehen werden. Dennoch, so könnte aus anderer Perspektive argumentiert werden, tangiert auch die Verteilung des Kuchens diesen nicht in seinem ,Kuchen-Sein“: Die einzelnen Stücke sind immer noch Teile desselben Kuchens und teilen exakt dessen Beschaffenheit. Dagegen verändern Handlungen wie Den Kuchen verbrennen lassen, auf den Boden fallen lassen die Beschaffenheit des Kuchens sehr wohl.

Sieht man sich die Vorkommen der Verbindung zur Verteilung bringen an, so zeigt sich freilich, dass in dieser Weise strittige Konkreta wie Kuchen gar nicht erst als Objekte vorkommen. Bei den einschlägigen Belegen, die DEREKo auswirft, 
handelt es sich jedenfalls durchgehend um Gegenstände, deren Verteilung keine Zustandsveränderung des Patiens involviert, vgl. (49). ${ }^{98}$

(49) Aufruf, Broschüre, Bundesabgaben, Gelder, Hirsche, Kohlsuppengrieß, Revanche (österr. für ,Entschädigung`), Lebensmittel, Flugblätter (4x), Produktivitätszuwachs, Summen, Superman-Puppen, Tombolapreise, Waren und Medikamente, 135 Laptops, 536 Derby-Star-Bälle, Geschenke, 1880 Euro, 2200 Euro, 470 Million Schilling, Paletten mit Lebensmitteln, Schirme, Schmiergeld, Staatsgelder, Tourismusbericht, Werbegeschenke, Wohlstand

Die genannten Objekte von zur Verteilung bringen verstoßen sicherlich nicht gegen den entsprechenden Constraint von bringen: Broschüren, Gelder oder Lebensmittel bleiben auch nach einer Verteilung als solche identifizierbar.

Insgesamt lässt sich zu den FVG mit bringen also festhalten, dass die Auswahl der Nomina stark beschränkt ist: Verb und Ereignisnomen müssen hinsichtlich ihrer Ereignisklasse sowie in der Art und Weise, wie die semantischen Rollen miteinander interagieren, übereinstimmen; andernfalls kann kein FVG mit bringen gebildet werden.

\subsection{FVG mit geben}

\subsubsection{Der Bestand der Nomina}

Während die Nomina mit bringen nicht auf Anhieb einer semantischen Klasse zuzuordnen waren, stellt sich der Fall bei den FVG der Struktur jmdm. N geben einfacher dar, da hier auf den ersten Blick drei deutlich unterscheidbare semantische Gruppen erkennbar sind. Die erste Gruppe, vgl. hier (50), umfasst Nomina, die einen Kommunikationsakt bezeichnen. ${ }^{99}$

(50) Anordnung, Anregung, Antwort, Auftrag, Aufklärung, Auskunft, Befehl, Einverständnis, Einwilligung, Empfehlung, Erklärung, Erläuterung, Erlaubnis, Genehmigung, Nachricht, Rat, Ratschlag, Tipp, Versprechen, Zusage, Zusicherung, Zustimmung

98 Abfrage 18.11.2015 (Suche nach ,zur Verteilung \&bringen“ direkt nacheinander).

99 Zum Artikelgebrauch im Einzelnen s. Kapitel 6.3. 
Die zweite Gruppe enthält Ereignisnominalisierungen, die eine - wie auch immer geartete - Einwirkung eines Agens auf eine Person bezeichnen, vgl. (51).

(51) Klaps, Schlag, Tritt, Kuss, Rüffel, Schlag, Stoß, Ohrfeige

Neben den umfangreicheren Gruppen (50) und (51) gibt es noch einen kleineren Bestand von Fügungen, die sich zumindest grob einer semantischen Klasse ,positive Bereicherung` o. ä. zuordnen lassen, vgl. (52).

(52) Beistand, Unterricht, Garantie, Hilfe/Hilfestellung, Unterstützung

Hier scheinen die Fügungen mit bringen aber häufig Formulierungsalternativen für Fügungen mit FVG leisten zu sein. Bei einzelnen dieser Fügungen liegt auch eine Präferenz für ein Possessivpronomen vor (seinen Beistand, seine Garantie, seine Unterstützung geben).

FVG aller drei Gruppen werden überwiegend mit einem Dativobjekt konstruiert, vgl. (53).

(53) a. Susi gab Peter einen Auftrag.

b. Der Professor gab dem Studenten eine Erklärung für sein Verhalten.

c. Der Professor gab dem Studenten keine Unterstützung.

In Einzelfällen ist auch eine Konstruktion mit einer an-PP möglich, wie die Beispiele in (54) zeigen. (Der syntaktische Status dieser PP wird in Kapitel 6.1 diskutiert.)

(54) a. Susi gab Peter einen Tipp./Susi gab einen Tipp an Peter.

b. Der Vater gab seinem Kind einen Rat./Der Vater gab einen Rat an sein

Teilweise erscheint eine an-PP aber fragwürdig oder sie erzwingt eine andere Bedeutung des Nomens, wie die Beispiele in (55) belegen.

(55) a. Der Vater gibt dem Kind eine Antwort./?Der Vater gibt eine Antwort an das Kind.

b. Peter gab Susi die Genehmigung./Peter gab die Genehmigung an Susi.

Die Variante mit PP in (55b) ist am ehesten akzeptabel, wenn man davon ausgeht, dass es sich bei Genehmigung um einen realen Gegenstand, d. h. in diesem Fall 
um ein Genehmigungsschreiben, handelt. Die an-PP scheint also eher eine konkrete Bewegung im Raum zu kodieren, die Konstruktion mit Dativargument dagegen einen Transfer an einen Rezipienten (zur Rezipienten-Rolle Primus 2012: 44f.).

\subsubsection{Die Bedeutung von geben}

Mit dem zuletzt angesprochenen Gegensatz von PP und Dativargument ist auch ein viel beachtetes Problem der Semantik von geben berührt. Aufgrund dieser Konstruktionsalternative, wird in zahlreichen Beschreibungsansätzen eine Polysemie des Verbs geben postuliert: In der Konstruktion mit Dativobjekt sei von einem Verb des Besitzwechsels auszugehen, in der Verbindung mit PP liege hingegen ein Transportverb vor (vgl. Pinker 1989: 82; Hale/Keyser 2002: 177; Wunderlich 2006: 115), vgl. (56). Der Polysemiehypothese zufolge stehen also eine ,caused-possession-' und eine ,caused-motion'-Lesart von geben einander gleichberechtigt gegenüber (Termini nach Levin 2008: 285).

(56) a. Der Junge gab dem Mädchen das Buch. caused possession:, $\mathrm{x}$ causes y to have $\mathrm{z}^{\text {‘ }}$

b. Der Junge gab das Buch an das Mädchen. caused motion: $\mathrm{x}$ causes $\mathrm{y}$ to be at $\mathrm{z}^{6}$

Rappaport Hovav/Levin (2008) vertreten dagegen einen monosemischen Ansatz, dem zufolge engl. give sowohl in der Konstruktion mit Dativobjekt als auch in der Verbindung mit PP als, caused possession' $\mathrm{zu}$ beschreiben ist.

Im Hinblick auf dt. geben sprechen die Argumente eher für die Monosemiehypothese. Einen Hinweis darauf bieten die Beispielsätze in (57), welche gleichermaßen die Folgerung in (58) zulassen.

(57) a. Der Junge gibt dem Mädchen das Buch.

b. Der Junge gibt das Buch an das Mädchen.

Das Mädchen hat das Buch.

Da sowohl für das mit Dativobjekt als auch für das mit PP konstruierte geben gefolgert werden kann, dass ,x y hat', liegt es in der Tat nahe, die ,caused-possession'-Lesart für beide syntaktischen Realisierungen als grundlegend anzunehmen. Dass der syntaktischen Variation semantische Unterschiede entsprechen 
können, ist damit freilich nicht grundsätzlich in Abrede gestellt. Für geben als FV kann die Frage nach der Alternation Dativobjekt/PP und damit einhergehenden semantischen Differenzierungen jedoch vernachlässigt werden, da, wie oben dargelegt, die Konstruktion mit Dativargument für das FVG ohnehin der Normalfall ist.

Die Konzentration der Forschung auf die syntaktische Alternation zwischen Dativ und PP hat dazu geführt, dass wesentliche Aspekte der lexikalischen Semantik des Verbs bisher nur unzureichend in den Blick gekommen sind. So kommen die gängigen Bedeutungsbeschreibungen über eine Paraphrase des Typs ,x causes y to have $z^{\prime}$, s. o. (56a), oder ,x causes y to receive z' (z. B. bei Goldberg 1995: 75; Levin 2008: 285; Proost 2014: 36) nicht hinaus. Problematisch an diesen Bedeutungsangaben ist, dass die wesentlichen Bestandteile der Beschreibung die Prädikate HAVE und RECEIVE - nicht oder nur ungenügend expliziert sind. Dies gilt übrigens auch für Dekompositionsstrukturen, die anstelle natürlichsprachlicher Explikatoren auf abstrakte Konzepte wie „POSS“ zurückgreifen (vgl. „ACT (x) \& BECOME POSS (y,z)“ bei Wunderlich 2000: 250; vgl. auch Winhart 2005: 169). Meinunger (2006: 90) hat das Prädikat „POSS“ insofern etwas expliziter gemacht, als er es aus einer lokativen Relation „BE AT“ herleitet (s. dazu auch Abschnitt 5.2.3). Auf diesem Vorschlag aufbauend könnte die Bedeutung von geben zunächst wie in (59) beschrieben werden.

$$
\text { geben: } \forall \mathrm{x} \forall \mathrm{y} \forall \mathrm{z}[\operatorname{GEBEN}(\mathrm{x}, \mathrm{y}, \mathrm{z}) \rightarrow \operatorname{CAUSE}(\mathrm{x}, \operatorname{BECOME}(\operatorname{BE} \mathrm{AT}(\mathrm{y}, \mathrm{z})))]
$$

Die Dekomposition in (59) ist freilich mit weiteren Eigenschaften anzureichern, da sie in dieser Form auch für caused-motion-Ereignisse gelten kann, vgl. etwa bringen in (29). ${ }^{100}$ Im Unterschied zu Bewegungsereignissen liegt bei geben jedoch kein Pfad vor, wie (60) zeigt.

(60) a. ${ }^{\star}$ Sie gab ihm das Buch von der Küche zum Wohnzimmer.

b. * Sie gab ihm das Buch entlang des Tisches. ${ }^{101}$

100 Jackendoff (1990: 135) analysiert engl. give dementsprechend mit Hilfe eines Primitivs GO. 101 In diesem Fall ginge allenfalls Sie gab ihm das Buch über den Tisch (besser noch mit reichen). Über drückt hier aber kaum einen Pfad aus, sondern eher ein potentielles Hindernis im Raum. 
Ein weiterer Unterschied besteht darin, dass die z-Position auf belebte Partizipanten beschränkt ist: Man kann nur jemandem etwas geben. Die Bedeutung von geben wird daher wie in (61) modelliert:
a. geben: $\forall \mathrm{x} \forall \mathrm{y} \forall \mathrm{z}$ [GEBEN' $(\mathrm{x}, \mathrm{y}, \mathrm{z}) \rightarrow \operatorname{CAUSE}(\mathrm{x}, \operatorname{BECOME}(\mathrm{BE} A \mathrm{AT}(\mathrm{y}, \mathrm{z})))$ ]
b. $\forall \mathrm{x} \forall \mathrm{y} \forall \mathrm{z} \forall \mathrm{e}$ [geben' $(\mathrm{x}, \mathrm{y}, \mathrm{z}, \mathrm{e}) \rightarrow \operatorname{BELEBT}(\mathrm{z})$
$(\mathrm{z}) \& \neg \exists \mathrm{p}(\operatorname{PATH}(\mathrm{p}, \mathrm{e}))]$

\subsubsection{Geben und die Beschränkungen in der Kombinierbarkeit}

\subsubsection{Energietransfer}

Die Ereignisse, die die Nomina in (51) bezeichnen, enthalten einen Energietransfer von einer „energy source“ zu einem „energy sink“ (Langacker 1991: 283; vgl. das Konzept des „transfer of activity“ bei Hopper/Thompson 1980: 251f.). Dieser Energietransfer resultiert jedoch nicht in einem veränderten Zustand des EnergieEmpfängers, wie er etwa bei Das Kind zerbrach die Tasse vorliegt. Dies zeigt sich daran, dass die einschlägigen Testformulierungen für das Vorliegen eines veränderten Resultatszustands (s. o. [45]) kein durchgehend gutes Ergebnis für die Basisverben von (51) liefern; zerbrechen mündet nach Ausweis von (62a) dagegen sehr wohl in einen Zustandswechsel des Patiens.

(62) a. Die Tasse ist jetzt zerbrochen.

b. 'Hans ist jetzt gestoßen.

c. \#Hans ist jetzt geküsst.

d. 'Hans ist jetzt unterstützt.

Auch wenn der in (62b)-(62c) vorliegende Energietransfer keine dauerhafte und einschneidende Zustandsveränderung beim Empfänger herbeiführt, so kann man doch davon ausgehen, dass dieser Transfer immerhin eine temporär vorhandene Spur hinterlassen kann: Wenn jemand gestoßen wird, vgl. (62b), gibt es möglicherweise eine Beule, einen blauen Fleck oder Ähnliches. Um die Ereignisse in (51) von den ,echten' Zustandsveränderungen wie zerbrechen abzuheben, könnte man hierfür ad hoc den Begriff der ,ephemeren Zustandsveränderung، einführen. Orientiert man sich an der Verbklassifikation von Levin/ Rappaport Hovav (1991: 132, 135), wären diese Verben als „verbs of impact by contact“ zu klassifizieren und von den „change-of-state verbs“ zu trennen. Fillmore (1970: 130) unterscheidet hier in analoger Weise zwischen „surface-contact 
verbs“ sowie „change-of-state verbs“ bzw. - nach den typischen Klassenvertretern - zwischen „verbs of hitting“ und „verbs of breaking“ (danach ist hier im Folgenden auch vom „schlagen-Typ“ und vom „,brechen-Typ“ die Rede; zu diesen Verbklassen weiter Jackendoff 1990: 110).

Dass das Merkmal ,Kontakt‘, welches Fillmore (1970) und Levin/Rappaport Hovav (1991) als klassenkonstituierend für Verben des schlagen-Typs ansehen, in der Tat eine wichtige semantische Eigenschaft der Ereignisse in (51) bildet, zeigt sich auch daran, dass am Energie-Empfänger meist jeweils eine (als PP realisierte) Kontaktzone identifizierbar ist: jemanden gegen das Schienbein treten, auf die Wange küssen, gegen die Brust schlagen.

In der semantischen Beschreibung kommt der Aspekt der ephemeren $\mathrm{Zu}$ standsveränderung bzw. des Kontakts dadurch zum Tragen, dass hier ein Prädikat „BECOME (BE AT)“ anstelle von „BECOME (STATE)“, welches für die Verben mit prototypischer Zustandsveränderung bzw. die „change-of-state-verbs“ im Sinne von Fillmore (1970: 130) einschlägig ist. In einer semantischen Analyse dieses Verbtyps ist das y-Argument dabei mit der Energie gleichzusetzen, die von $\mathrm{x}$ an den Rezipienten z übergeht und auf diesen einwirkt. Die semantische Analyse eines ephemeren Zustandsveränderungsverbs ist damit der Struktur von geben sehr ähnlich, vgl. (63) zu schlagen (hier mit einer manner-Angabe „BY HANDBEWEGUNG“).

schlagen: $\forall \mathrm{x} \forall \mathrm{y} \forall \mathrm{z}[$ schlagen'( $\mathrm{x}, \mathrm{y}, \mathrm{z}) \rightarrow \operatorname{CAUSE}(\mathrm{x}, \operatorname{BECOME}(\mathrm{BE}$ AT $(\mathrm{y}, \mathrm{z})))$ by HANDBEWEGUNG (x)]

Schlagen wäre dementsprechend $\mathrm{zu}$ paraphrasieren als „x bewirkt, dass eine Energie y bei $z$ ist, indem $x$ eine Handbewegung ausführt“. Zu überlegen ist allerdings, ob die y-Position in diesem Fall nicht einfacher mit Hand besetzt sein sollte: „x bewirkt, dass die Hand (y) bei z ist“. In diesem Fall läge allerdings ein bloßes Berührungsverb vor (zur Klasse der „,verbs of touching“ Jackendoff 1990: 106-112). Eine solche Paraphrase beschreibt in der Tat eher ein Verb wie berühren, das zwar einen Kontakt, dafür jedoch keinen oder keinen nennenswerten Energietransfer bzw. eine daraus resultierende Zustandsveränderung beim Patiens (und sei sie minimal) ausdrückt.

Wenn die Kompatibilität von geben mit den Energietransfer-Ereignissen in (51) wesentlich darin begründet ist, dass in beiden Fällen ein Transfer ohne dauerhafte Zustandsveränderung am Patiens vorliegt, so wird verständlich, weshalb Verben wie schneiden oder kratzen nicht als Basis eines FVG-Nomens mit geben möglich sind ( ${ }^{\star} j m d m$. einen Schnitt/Kratzer geben). Diese Verben mögen zwar insofern eine gewisse Ähnlichkeit mit dem schlagen-Typus aufweisen, als auch hier 
ein Kontakt vorliegt. Bei näherem Hinsehen ist schneiden bzw. kratzen jedoch kaum als ephemeres Zustandsveränderungsverb zu beurteilen, da diese Handlungen in jedem Fall ein sichtbares Resultat am Patiens hinterlassen. Nach Vollzug einer schneiden-Handlung kann man feststellen: Da ist ein Schnitt auf der Haut. Bei schlagen oder treten ist eine vergleichbare Feststellung nicht möglich ( ${ }^{\star}$ Da ist ein Schlag/Tritt auf der Haut). Im Unterschied zu den Verben, die in FVG mit geben akzeptabel sind, liegen bei Verben des schneiden-Typs somit sichtbar veränderte Resultatszustände eines Patiens vor.

Für die Besetzung der N-Position ist auch mindestens eine weitere Eigenschaft von geben relevant. So kann die Einschränkung in (61b), der zufolge geben kein Pfad-Argument enthält, gut erklären, weshalb Verben wie beschießen, bewerfen bzw. schießen auf, werfen auf nicht in einem entsprechenden FVG auftreten. Bei diesen Verben ist zwar durchaus ein Transfer von Energie an einen Empfänger gegeben. Da jedoch ein Pfad zwischen Energiequelle und -empfänger besteht (Er schoss von der Mauer aus auf den Mann), wären entsprechende Nominalisierungen nicht mit der Bedeutung von geben vereinbar. Eine Fügung wie in (64) ist folglich inakzeptabel.

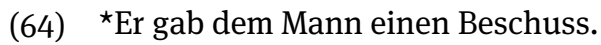

Ornativa (verschmutzen, bemalen, einölen) wiederum enthalten zwar ebenfalls kein Pfad-Argument, sie sind dafür im Gegensatz zu treten, schlagen oder küssen jedoch durch einen sichtbar veränderten Resultatszustand gekennzeichnet, wie (66) belegt (s. auch Abschnitt 5.2.1).

(65) Nach der Behandlung war der Teppich vollkommen verschmutzt.

Die Nichtakzeptabilität eines geben-FVG, vgl. (66), ist vor diesem Hintergrund verständlich.

(66) ${ }^{\star}$ Die Kinder gaben dem Teppich eine Verschmutzung.

Auffällig an den Nomina in (51) ist, dass die Partizipanten überwiegend belebt sind. Bei den Kommunikationsereignissen in (50) versteht es sich von selbst, dass hier nur belebte Partizipanten involviert sein können, da Kommunikation belebte Partizipanten voraussetzt; aber auch für die Nomina der Gruppe (51) besteht offenbar ebenfalls eine Präferenz für einen belebten Partizipanten. (67a) und (68a), die einen belebten Rezipienten enthalten, sind gegenüber (67b) und (68b) jedenfalls deutlich akzeptabler: 
(67) a. Susi gab Peter einen Tritt.

b. 'Susi gab dem Tisch einen Tritt (vs. Susi trat gegen den Tisch).

(68) a. Susi gab Peter einen Kuss.

b. ?Susi gab dem Brief einen Kuss.

Auch eine Stichprobe in DEREKo ergibt für die Kombination von Tritt mit unmittelbar folgendem geben (in allen Formen) unter 152 Treffern nur 18 für ein unbelebtes Objekt. ${ }^{102}$ Diese Kombination ist vor allem deshalb schwierig - so ist $\mathrm{zu}$ vermuten -, weil in diesem Fall keine Spezifikation der Kontaktzone möglich ist, wie (69b) zeigt:

(69) a. Susi gab Peter einen Tritt gegen das Scheinbein.

b. ^Susi gab dem Tisch einen Tritt gegen die Kante.

In diesem Punkt besteht somit ebenfalls eine Übereinstimmung mit geben, dessen z-Partizipant im Normalfall ein belebter Rezipient ist, s. (61b).

\subsubsection{Kommunikationsereignisse}

Dass Kommunikationsereignisse in der N-Position von FVG mit geben auftreten, wie die Beispiele der Gruppe (50) zeigen, erscheint einleuchtend, da Kommunikation in der Regel als Transfer vom Information konzeptualisiert ist: Man kann Nachrichten übermitteln, eine Botschaft überbringen, weitergeben oder zurückhalten usw. Diese Parallelität von Kommunikation und Transfer wird in der kognitiven Linguistik in Form einer metaphorischen Projektion KoMMUNIKATION IST TRANSFER beschrieben, vgl. Lakoff (1987: 450). Die semantische Struktur von Kommunikationsereignissen ist mit einer Struktur wie in (61) daher gut beschrieben. In dieser Struktur sind dann bloß die Argumentstellenbesetzungen in besonderer Weise spezifiziert: y steht nicht für einen konkreten Gegenstand, der an einen belebtes z gelangt, sondern für eine Information. Nicht nur das Bedeutungspostulat ,z ist belebt“ besitzt hier Gültigkeit, auch der Ausschluss des Pfad-Arguments, der die Struktur in (61) entscheidend von Bewegungsereignissen abhebt, ist für die Domäne Kommunikation einschlägig, wie die fragwürdigen Formulierungen in (70) zeigen.

102 Letzter Zugriff am 06.04.2021. 
(70) a. 'Er befiehlt von seinem Zimmer in die Küche.

b. 'Er befiehlt den Flur entlang.

Die Tatsache, dass Ableitungen von Verben wie schreien, brüllen, wimmern, die primär ein Lautereignis und allenfalls sekundär einen Kommunikationsakt bezeichnen, nicht in geben-FVG vorkommen, erscheint vor dem Hintergrund der Beschreibung in (61) verständlich. Ein Verb wie schreien wird man kaum mit einer solchen Dekomposition erfassen können; stattdessen liegt eher ein einfaches Prädikat „АCT (x)“ oder „DO (x)“ vor. Verben dieses Typs sind folglich mit geben semantisch nicht kompatibel. Hier ist lediglich die Konstruktion (einen Schrei, Laut, eine Äußerung) von sich geben möglich, die keinen Transfer an einen Rezipienten ausdrückt, sondern den Ausgangspunkt der Lautemission fokussiert.

Im Hinblick auf die Frage, welche Kombinationen von geben mit einer Nominalisierung aus welchem Grund möglich oder unmöglich sind, ist an dieser Stelle noch einmal etwas ausführlicher auf die FVG einzugehen, die Kommunikationsereignisse versprachlichen, s. o. (50). Da Kommunikation, wie erwähnt, grundsätzlich als Transfer von Information beschrieben werden kann, wäre eigentlich zu erwarten, dass hier kaum nennenswerte Einschränkungen der Kombinierbarkeit mit geben in FVG bestehen. Gleichwohl gibt es mit bitten und verbieten mindestens zwei Kommunikationsverben, die als Nominalisierungen in einem geben-FVG nicht akzeptabel sind.

*jmdm. eine Bitte geben/^ein Verbot geben

Dies ist umso erstaunlicher, als mit jmdm. einen Befehl geben und jmdm. eine Erlaubnis geben semantisch eng verwandte FVG existieren. Eine Erklärung für die Nichtakzeptabilität von *jmdm. eine Bitte geben könnte man darin sehen, dass bitten mit Akkusativobjekt, nicht mit Dativobjekt steht wie fast alle Kommunikationsverben, die ein geben-FVG neben sich haben. Indes wird dieser Faktor kaum ausschlaggebend sein, da etwa auch aufklären und anordnen trotz eines fehlenden Dativarguments ein FVG bilden. Den Ausschluss von *jmdm. ein Verbot geben würde dies ohnehin nicht plausibel machen, da jmdm. etwas verbieten ja vollkommen gängig ist.

Eine weitere Erklärungsmöglichkeit bestünde in der Annahme einer Art Synonymenflucht oder Blockierung: Die Fügungen in (71) wären demzufolge deshalb nicht akzeptabel, weil es die Synonyme eine Bitte aussprechen und ein Verbot aussprechen gibt (die beide übrigens auch als FVG im hier vertretenen Sinne gelten können). Irgendein sprachökonomisches Prinzip, demzufolge ein Konzept 
nicht durch zwei oder mehrere FVG kodiert werden kann, ist jedoch offensichtlich nicht existent, da z. B. neben eine Bitte aussprechen auch das synonyme FVG eine Bitte vorbringen besteht oder zu Mitteilung machen auch die (freilich seltenere) Ausdrucksalternative Mitteilung geben belegbar ist. ${ }^{103}$

Eine Erklärung für die Nichtakzeptabilität von *ein Verbot geben und *eine Bitte geben ist möglicherweise eher bei semantischen Eigenschaften der zugrundeliegenden Verben zu suchen. Ein Grund könnte darin liegen, dass jmdm. etwas verbieten zwar ein Transfer von Information ist, der Empfänger einer Nachricht ,du sollst nicht $\mathrm{P}^{\prime}$ jedoch nicht in der Weise von der Nachricht profitiert wie der Empfänger der Kommunikationsereignisse jmdm. antworten, jmdm. etwas erklären oder jmdm. etwas raten. Verbieten wäre damit eher auf den Sender, d. h. auf denjenigen, der das Verbot ausspricht, fokussiert. Gleiches gälte dann auch für bitten/* eine Bitte geben: Gebeten zu werden ist für den Empfänger einer Bitte kein ,Zugewinn‘. Deshalb erscheint es naheliegend, dass das entsprechende FVG ein Verbot/eine Bitte aussprechen lautet und damit den Sender anstelle des Empfängers in den Mittelpunkt rückt. Dieser Erklärungsversuch lässt allerdings die Frage offen, weshalb jmdm. einen Befehl geben akzeptabel ist, da auch hier der Empfänger kaum von der übermittelten Information profitiert.

Eine zufriedenstellende Lösung hierfür kommt wohl erst in den Blick, wenn man weitere Fälle heranzieht. So hat Winhart (2005) auf eine vergleichbare Akzeptabilitätsidiosynkrasie bei den semantisch untereinander sehr ähnlichen Verben zustimmen und befürworten hingewiesen: Seine Zustimmung geben ist akzeptabel (ebenso wie seine Einwilligung und sein Einverständnis geben), *seine Befürwortung geben dagegen nicht, vgl. (72).

(72) a. Der Bürgermeister gibt seine Zustimmung zu dem Projekt.

b. ${ }^{\star}$ Der Bürgermeister gibt seine Befürwortung zu dem Projekt.

Winhart (2005: 167-169) führt diesen in der Tat erstaunlichen Unterschied zunächst auf die je eigenen semantischen Strukturen der Verben zustimmen bzw. befürworten zurück.

103 Vgl. das folgende Beispiel: „Zum Schluß der Versammlung erklärte Martens, daß er in zwei Jahren bei der nächsten Vorstandswahl nicht mehr kandidieren werde. Mit 70, sagte er, sei es genug und er wolle rechtzeitig Mitteilung geben, damit der Nachfolger genügend Zeit zur Einarbeitung habe“ (Rhein-Zeitung, 20.03.1997; Der Tierpark öffnet schon Karfreitag; Zugriff 06.04.2021). 

a. befürworten: $\lambda \mathrm{y} \lambda \mathrm{x} \lambda \mathrm{r}[\mathrm{DO}((\mathrm{x}, \mathrm{y}) \mathrm{r})]$
b. zustimmen: $\lambda y \lambda x \lambda e:[\operatorname{DO}((x) r), \& \operatorname{BEC}((\operatorname{POSS}((y, z) s))$ e)]

(Winhart 2005: 167 bzw. 169)

Von zustimmen unterscheidet sich befürworten Winhart zufolge ferner darin, dass es eine Proposition als Patiens aufweist. Daher setzt sie die spezifischere Notation (74) an, in der „p“ für „Proposition“ steht.

$$
\text { befürworten: } \lambda \mathrm{p} \lambda \mathrm{x} \lambda \mathrm{r}[\mathrm{DO}((\mathrm{x}, \mathrm{p}) \mathrm{r})]
$$

(Winhart 2005: 167)

In dieser besonderen Argumentstellenbesetzung sieht sie neben der abweichenden semantischen Grundstruktur einen weiteren Hinderungsgrund für die Verbindung von Befürwortung mit geben: „Befürworten ist [...] eine Relation eines Agens zu einer Proposition. Diese Relation kann nicht ,gegeben“ werden“ (Winhart 2005: 171).

Dass eine Proposition (bzw. die Relation zwischen Agens und Proposition) nicht ,gegeben' werden kann, ist jedoch nicht einzusehen. Zustimmen, das mit jmdm. seine Zustimmung geben ein FVG bildet, kann ebensogut als Relation zwischen einem Agens und einer Proposition beschrieben werden wie befürworten. Läge tatsächlich ein Unterschied zwischen befürworten und zustimmen im Sinne Winharts vor, wäre überdies zu begründen, weshalb die durch befürworten kodierte Relation nicht ,gegeben ' werden kann. Hierfür müsste ein übergeordneter Gesichtspunkt angeführt werden, der diese Beschränkung verständlich machen könnte.

Wenn das Vorhandensein einer Proposition nicht der entscheidende Faktor für die Inakzeptabilität von *Befürwortung geben darstellt, so verbliebe immerhin noch der von Winhart postulierte Unterschied in der semantischen Struktur von befürworten und zustimmen in (73a) bzw. (73b) als Erklärung für deren unterschiedliches Verhalten. Der Ansatz einer je eigenen Bedeutungsstruktur für beide Verben wirkt jedoch ad hoc und zirkulär. Es wird jedenfalls keine unabhängige Evidenz dafür angeführt, weshalb sich die Bedeutungsstruktur zweier so ähnlicher Verben in der angegebenen Weise unterscheiden sollte. Wenn man sagen kann Der Plan hat meine Zustimmung, nicht aber ${ }^{\star}$ Der Plan hat meine Befürwortung, so ist dies jedenfalls keine unabhängige Evidenz, da die haben-Fügung möglicherweise nur deshalb besteht, weil sie von der Existenz der jeweiligen geben-Fügung abhängig ist.

Der Grund für die hier vorliegenden Akzeptabilitätsverhältnisse wird somit sicherlich nicht oder nicht allein bei semantischen Eigenschaften der jeweiligen 
Verben, sondern vor allem bei syntaktischen Gegebenheiten zu suchen sein. Befürwortung auf der einen Seite sowie Zustimmung, Einwilligung und Einverständnis auf der anderen Seite sind nämlich durch die Art und Weise unterschieden, in der die Attribuierung erfolgt: Im Fall von Befürwortung ist nur ein Genitivattribut möglich, in den anderen Fällen hingegen nur eine attributive PP, vgl. (75) vs. (76).

(75) die Befürwortung des Plans/ ${ }^{\star} z u$ dem Plan/`für den Plan

(76) a. *die Zustimmung des Plans/die Zustimmung zu dem Plan

b. *die Einwilligung des Plans/die Einwilligung in den Plan

c. *das Einverständnis des Plans/das Einverständnis mit dem Plan

Die Nomina in (76) treten in FVG auf; Befürwortung, das nur durch eine NP im Genitiv attribuiert werden kann, hingegen nicht. Dies kann nun vor dem Hintergrund der Hypothese erklärt werden, dass ein Attribut zum Nomen in FVG auch als Satzglied reanalysierbar sein muss. In der Fügung ${ }^{\star} E r$ gibt die Befürwortung des Plans ist das Genitivattribut nicht als Satzglied interpretierbar, da genitivische Satzglieder - von wenigen Ausnahmen wie sich einer Sache bewusst werden usw. abgesehen - grundsätzlich nicht möglich sind. Eine Ergänzung durch eine PP wie im Fall von Zustimmung geben zu dem Plan ist in dieser Hinsicht unproblematisch, da die PP auch als Satzglied aufgefasst werden kann. Dass die PPs zu Nomina in FVG als Satzglieder aufzufassen sind, stellt jedenfalls die gängige Position der Forschung dar (s. dazu und zu einer weitergehenden Diskussion Kapitel 6.1).

Insgesamt wird man für die FVG mit geben festhalten können, dass die Auswahl der Nomina insgesamt gut von den Eigenschaften des Verbs geben her erklärbar ist. Zum Teil sind aber, wie zuletzt gezeigt, relativ detaillierte semantische und syntaktische Analysen notwendig, damit zugrunde liegende Regularitäten herausgearbeitet werden können.

\subsection{FVG mit machen}

\subsubsection{Der Bestand der Nomina}

Das Verb machen kommt in zahlreichen Fügungen vor, die als FVG im Sinne dieser Untersuchung gelten können, da sie eine Implikationsbeziehung zwischen Nominal- und Gesamtbedeutung aufweisen. Die einschlägigen Verbindungen können teilweise bestimmten semantischen Klassen zugeordnet werden, wie die - nicht erschöpfende - Aufstellung der Nomina in (77) zeigt. Teilweise fehlt 
aber auch die Möglichkeit einer Klassenzuweisung, vgl. die Untergruppe (77g). Ob die skizzierte Einteilung mehr als einen ersten heuristischen Wert hat, sei dahingestellt.

(77) a. physische Aktivitäten: Salto, Sprung, Schritt, Drehung, Übung, Verbeugung, Knicks, Geste, Bewegung, Handbewegung

b. Reise: Gang, Fahrt, Ausflug, Reise, Abstecher, Exkursion, Wallfahrt, Expedition, Flug, Tour, Trip

c. Rekreation: Pause, Urlaub, Ferien, Schläfchen, Party, Fest

d. Sprechakte: Andeutung, Anspielung, Aussage, Bemerkung, Anmerkung machen, jmdm. einen Vorschlag, eine Mitteilung, Witz, Scherz, Jux, Versprechungen (Pl.), Vorhersage, Prophezeiung, Vorwurf, Vorhaltungen, Kompliment(e)

e. psychische Zustände (reflexiv): Illusionen, Hoffnungen, Gedanken, Sorgen

f. geplante Handlungen: eine Messung, Untersuchung, Studie ${ }^{104}$

g. Diverse: Arbeit, Besuch, Beobachtung, Entdeckung, Fund, Gebrauch, Fortschritt, Rück schritt, Fehler, Versuch, Experiment, Jagd, Zugeständnisse

Viele dieser Bildungen bieten die Möglichkeit, ein Argument als PP anzuschließen, vgl. (78).

(78) a. einen Sprung ins Wasser machen

b. einen Ausflug an den See machen

c. einen Witz über den Opa machen

d. einen Besuch bei der Tante machen

Einige FVG mit machen enthalten Nomina, die keine Position für ein Argument bereitstellen, z. B. Pause oder Schläfchen. Umso mehr verwundert, dass Verbindungen wie eine Entdeckung, Beobachtung, einen Fund machen, deren Nomina sonst durchaus zwei Argumente haben, s. (79), innerhalb des FVG kein weiteres Argument vergeben, wie (80) demonstriert.

(79) a. die Entdeckung des Schatzes durch die Piraten

b. der Fund der Arche Noah durch den Archäologen

104 Für diese Fälle bietet sich durchführen als Synomym an. 
(80) a. *Susi macht eine/die Entdeckung des Schatzes.

b. *Susi machte den Fund der Arche Noah.

Dies liegt wie im Fall von *Befürwortung geben (s. o. 3.3.2) offenbar daran, dass der genitivische Anschluss keinen syntaktisch wohlgeformten Satz ergibt. Das genitivische Argument in (80) ist nur als Attribut zum Nomen, nicht aber als Komplement auf Satzebene analysierbar. Letzteres scheint aber wohl erforderlich $\mathrm{zu}$ sein (vgl. auch Kapitel 6.1).

\subsubsection{Die Bedeutung von machen}

Um mögliche Motivationen für die Auswahl der Nomina zu erkennen, ist, wie bei den anderen hier behandelten Funktionsverben auch, ein Blick auf die Bedeutung des Verbs machen nötig. Mit dem weiter unten noch zu behandelnden haben teilt machen die Eigenschaft, dass ein sehr breites Lesartenspektrum vorliegt. In Anlehnung an Chur (1996: 16) können die folgenden Verwendungen von machen (ohne Anspruch auf Vollständigkeit) unterschieden werden:

(81) a. einen Kuchen machen (,herstellen')

b. jmdm. Angst machen (,einen Zustand verursachen')

c. Tuut-tuut machen (,Geräusche hervorbringen')

d. große Augen/ein dummes Gesicht machen (,einen mimischen Ausdruck hervorbringen')

e. den wilden Mann machen (,darstellen')

f. das Bett/sich die Haare machen (,zurechtmachen')

g. Drei plus 3 macht 6/Das macht drei Euro (,als Summe ergeben, kosten`)

h. Was machen Sie da?/Ich kann es nicht genau sehen, aber sie macht etwas. (Proverb)

i. einen Weltstar aus jmdm. machen/jmdn. zu einem Weltstar machen (aus/zu-Alternation)

j. seine Hausaufgaben, seine Arbeit machen (,(eine Aufgabe) erledigen')

k. seine Hausaufgaben, seine Arbeit machen (,(eine Aufgabe) erledigen')

1. Yoga/Sport machen (,betreiben')

Wie Chur (1996: 16) mit Recht feststellt, wird das ohnehin sehr reiche semantische Spektrum noch um zahlreiche idiomatische Verwendungen des Verbs erweitert 
(sich zum Affen machen, Sie macht das Rennen, sich nichts aus etwas machen, machs gut!, den Unterschied machen usw.). ${ }^{105}$ Auch wenn man einzelne der von Chur herausgearbeiteten Lesarten unter einer allgemeineren Bedeutungsposition zusammenfassen könnte - etwa (81a)-(81d) unter eine Lesart ,hervorbringen“ -, bleibt es dabei, dass mit machen ein stark polysemes Verb vorliegt.

Die Suche nach einem gemeinsamen Nenner dieser Bedeutungen ist freilich nicht aussichtslos: Machen bezeichnet in jedem Fall, sofern das Subjekt als belebt zu klassifizieren ist, eine Tätigkeit, die bewusst, zielgerichtet und kontrolliert ausgeführt wird. Als Bedeutung kann daher schlicht das Prädikat Do (x, y) angesetzt werden.

Dass machen mit einem Prädikat Do (x, y) gleichzusetzen ist, wird auch durch die Proverblesart in (81h) bestätigt. Wenn man nach der allgemeinsten Bedeutung von machen sucht, ist das Proverb jedenfalls ein guter Kandidat, da es als Stellvertreter für eine ganze Reihe möglicher Verbalereignisse eingesetzt werden kann; es ist somit per se unterspezifiziert. ${ }^{106}$ Dies zeigt der Testrahmen in (82), der einen durch nämlich eingeleiteten explizierenden Folgesatz enthält.

(82) a. Sie macht etwas, nämlich einen Aufsatz schreiben.

b. Sie macht etwas, nämlich Erdbeeren pflücken.

c. Sie macht etwas, nämlich laufen.

Machen in der Proverblesart ist zwar semantisch sehr allgemein, es ist jedoch nicht vollkommen beliebig, ${ }^{107}$ wie die eingeschränkten Kombinationsmöglichkeiten in den Beispielen in (83) zeigen.

105 Idiomatische Züge trägt auch das häufig als Kausativum klassifizierte machen in jmdn. glauben machen, dass (...), jmdn. lachen/weinen machen (vgl. Chur 1996: 16). Es handelt sich nur um wenige Verbindungen, in denen ein solches machen mit kausativer Funktion vorliegt. Aufgrund der stark eingeschränkten Kombinationsmöglichkeiten, die machen hier aufweist, ist eher von einem Phraseologismus auszugehen.

106 Selbst auf die an sich schon relativ abstrakte faktitive Lesart (j) kann machen sich als Stellvertreter beziehen: Was macht er gerade? Er macht die Wand grün.

107 So die Auffassung von Hale/Keyser (2002: 93), die do und make als „verbs without any semantic component" beschreiben. Als Begründung wird angeführt, dass diese Verben nicht ohne Objekt auftreten können (z. B. *Hans tut, ${ }^{\star}$ Hans macht). Daher könnten sie keine semantisch leeren Komplemente lizensieren. Dies ist ein zentraler Theoriebaustein in Hale/Keysers Ansatz, der unergative Verben wie dance oder cry auf eine verdeckte transitive Struktur mit „light verb“ zurückführt; hierzu auch Kapitel 8.1.2. 
(83) a. 'Sie macht etwas, nämlich schlafen.

b. * Sie macht etwas, nämlich an Kopfschmerzen leiden.

c. *Sie macht etwas, nämlich die Mathe-Aufgabe verstehen.

d. *Sie macht etwas, nämlich ankommen.

Machen kann somit nur mit einem agentiven Verb im explizierenden Folgesatz stehen. Nicht-agentive Verben wie die Achievements verstehen, ankommen sowie der Zustand leiden sind in dieser Position nicht möglich. Daraus lässt sich ableiten, dass machen selbst als agentives Verb Do (x, y) zu klassifizieren ist.

\subsubsection{Machen und die Beschränkungen in der Kombinierbarkeit}

Nachdem die Bedeutung des FV eingegrenzt ist, muss in einem zweiten Schritt nach den semantischen Eigenschaften der Nomina gefragt werden. Folgt man der zu Beginn des Kapitels formulierten Arbeitshypothese, nach welcher das dem Nomen zugrundeliegende Verb und FV semantisch grundsätzlich übereinstimmen, wären die Nomina in (77) zunächst darauf hin zu befragen, ob hier ebenfalls agensgesteuerte Ereignisse gegeben sind. Als Verfahren, welches das Vorliegen einer Agenssteuerung des Ereignisnomens evident machen kann, sei hier ein Test gewählt, der auf die für Agentivität wesentlichen Eigenschaften der Kontrolle zielt. Mit Kontrolle ist die Fähigkeit des Agens gemeint, das Ereignis selbstbestimmt auszulösen bzw. zu unterbinden und gegebenenfalls auch abzubrechen (vgl. Primus 2002: 3; 2012: 18). Aus der Kontrolle des Agens über das Ereignis ergibt sich als weitere Agenseigenschaft in der Regel auch dessen Verantwortung für das Zustandekommen des Ereignisses bzw. für die Art und Weise, in der es stattfindet (zu Kontrolle und Verantwortlichkeit als Agenseigenschaften vgl. neben Primus 2002: 3 auch Brennenstuhl 1982: 89f.).

Das Gegebensein der Eigenschaft Kontrolle sei im Folgenden durch Tests erfragt, die die Möglichkeit, das Ereignis zu unterbinden bzw. abzubrechen, zum Gegenstand haben: Susi unterlässt N, Susi bricht N ab ist hierfür ein geeigneter Test. Für die Eigenschaft Verantwortlichkeit soll ein Muster Susi entschuldigt sich für Det/Poss N als Testrahmen verwendet werden. Beide Tests sind insofern als aussagekräftig zu bewerten, als offensichtlich nicht-agensgesteuerte Nominalereignisse wie z. B. Schmerz, Leid, Freude nicht oder nur sehr schwer in die betreffenden Formulierungen eingefügt werden können: 
(84) a. *Susi unterlässt ihren Schmerz, ihr Leid, ihre Freude.

b. * Susi bricht ihren Schmerz, ihr Leid, ihre Freude ab.

c. 'Susi entschuldigt sich für ihren Schmerz, ihr Leid, ihre Freude.

Wendet man die vorgeschlagenen Tests auf die Nomina in (77) an, ergibt sich folgendes Bild:

physische Aktivitäten:

- Sie unterlässt den Salto, Sprung, Schritt, die Drehung, die Übung, Verbeugung, den Knicks, die Geste, Bewegung, Handbewegung

$\rightarrow$ Handlungskontrolle

- Sie entschuldigt sich für den Salto, Sprung, Schritt, die Drehung, die Übung, Verbeugung, den Knicks, die Geste, Bewegung, Handbewegung

\section{$\rightarrow$ Verantwortlichkeit}

(86) Reise:

- Sie unterlässt den Gang, die Fahrt, den Ausflug, die Reise, den Abstecher, die Exkursion, die Wallfahrt, die Expedition, den Flug, die Tour, den Trip

$\rightarrow$ Handlungskontrolle

- Sie entschuldigt sich für den Gang, die Fahrt, den Ausflug, die Reise, den Abstecher, die Exkursion, die Wallfahrt, die Expedition, den Flug, die Tour, den Trip

$\rightarrow$ Verantwortlichkeit

(87) Rekreation:

- Sie bricht die Pause, den Urlaub, die Ferien, (?)das Schläfchen, ${ }^{108}$ die Party, das Fest ab

$\rightarrow$ Handlungskontrolle

108 In diesem Zusammenhang ist besonders aufschlussreich, dass die Fügung Schläfchen machen akzeptabel ist, `Schlaf machen jedoch nicht. Dies kann genau damit begründet werden, dass Schläfchen als ein zeitlich begrenzter, nicht besonders tiefer und in den Tagesablauf eingebauter Erholungsschlaf einer gewissen Kontrolle durch den Partizipanten insofern nicht ganz entzogen ist, als der Ereignisbeginn von Schläfchen bewusst und willentlich herbeigeführt und im Normalfall ein Ende eingeplant wird. Dass Schläfchen Kontrolle involviert, Schlaf hingegen nicht, zeigt sich auch daran, dass eine Äußerung 'Ein Schläfchen übermannte ihn gegenüber Schlaf übermannte ihn als fraglich bzw. humoristisch einzustufen ist. 
- Sie entschuldigt sich für die Pause, den Urlaub, die Ferien, das Schläfchen, die Party, das Fest

\section{$\rightarrow$ Verantwortlichkeit}

(88) Sprechakte:

- Sie unterlässt die Andeutung, Anspielung, Aussage, Bemerkung, Anmerkung, den Vorschlag, die Mitteilung, die Versprechungen, die Vorhersage, die Prophezeiung, ?den Vorwurf, ?die Vorhaltungen, das Kompliment

\section{$\rightarrow$ Handlungskontrolle}

_ $\quad$ Sie lässt den Vorwurf, die Vorhaltungen fallen

\section{$\rightarrow$ Handlungskontrolle}

- Sie entschuldigt sich für die Andeutung, Anspielung, Aussage, Bemerkung, Anmerkung, den Vorschlag, die Mitteilung, die Versprechungen, die Vorhersage, die Prophezeiung, den Vorwurf, die Vorhaltungen, das Kompliment

$\rightarrow$ Verantwortlichkeit

(89) psychische Zustände (reflexiv):

- Lass die Illusionen, Hoffnungen, Gedanken, Sorgen

\section{$\rightarrow$ Handlungskontrolle}

- 'Sie entschuldigt sich für ihre Illusionen, Hoffnungen, Gedanken, Sorgen

\section{$\rightarrow$ ?'Verantwortlichkeit}

(90) Diverse:

- Sie unterbricht ihren Besuch, ihre Beobachtung der Pinguine, ihren Versuch, ihr Experiment

\section{$\rightarrow$ Handlungskontrolle}

- Sie entschuldigt sich für ihren Besuch, ihre heimliche Beobachtung der Pinguine, ihre Rückschritte, ihren Fehler, ihren Versuch, ihr Experiment, ihre Entdeckung

\section{$\rightarrow$ Verantwortlichkeit}

Als Ergebnis dieses Tests kann festgehalten werden, dass alle Nomina in (77) entweder die Agenseigenschaft Handlungskontrolle oder die Agenseigenschaft Verantwortlichkeit aufweisen - in den meisten Fällen liegen sogar beide vor. Damit können die hier genannten Nominalereignisse als grundsätzlich agensgesteuert angesehen werden, auch wenn ein Prototypikalitätsgefälle etwa zwischen der Agentivität von Bewegung und z. B. der von Schläfchen vorauszusetzen ist: Schläfchen ist aber immerhin die bewusst eingelegte Pause, für die man sich z. B. im 
Unterschied zum Nachtschlaf als unverzichtbarem Teil des Biorhythmus durchaus auch entschuldigen kann. Auch die psychischen Zustände in (89) sind selbstverständlich nicht in prototypischer Weise agensgesteuert. Handlungskontrolle liegt aber bis zu einem gewissen Grad durchaus vor. Damit gilt alles in allem, dass die Agenssteuerung des Nominalereignisses und die Agentivität von machen einander entsprechen.

Wenn man festhalten kann, dass das FV machen nur mit agensgesteuerten Ereignissen kompatibel ist, so stellt sich indes die Frage, weshalb es bei der insgesamt großen Zahl agentiver Verben bzw. agensgesteuerter Ereignisse doch nur eine relativ kleine Gruppe entsprechender FVG gibt. So liegen nicht wenige agensgesteuerte Ereignisnomina vor, die mit machen unverträglich zu sein scheinen. Dies gilt insbesondere für deverbale Nomina, die auf Accomplishments beruhen, vgl. (91).

(91) a. 'Ich mache die Räumung meiner Wohnung.

b. \#Ich mache die Reinigung des Fußbodens.

c. ${ }^{\star}$ Der Pianist macht das Spiel der Sonate.

d. ${ }^{\star}$ Das Kind macht die Verspeisung des Essens.

e. \#Ich mache den Umzug nach Berlin.

f. \#Die Sekretärin macht die Vernichtung der Akten.

Auch die Liste der mit machen kompatiblen NPs in (77) enthält keine Ereignisse, die Accomplishment-Charakter besitzen. Hier handelt es sich überwiegend um punktuelle bzw. iterative Ereignisse (Sprung, Bewegung, Salto, Satz, Schritt) oder um durative Ereignisse (Gang, Spaziergang, Reise, Fahrt, Pause, Urlaub, Besuch, Fortschritt). Der Grund dafür, dass Nominalereignisse mit Accomplishment-Charakter nicht in machen-FVG auftreten, ist in einer wichtigen Eigenschaft von Accomplishments zu sehen. Accomplishments verbinden die Eigenschaften Telizität und Durativität. Es handelt sich um ein zielgerichtetes Ereignis, das eine messbare Dauer bis zu seinem Abschluss einnimmt (Tenny/Pustejovsky 2000: 5). Dieses Ereignis zeichnet sich grundsätzlich durch die inkrementelle „Abarbeitung“ seines Patiens aus: In einem Syntagma wie den Apfel essen ist der Objektreferent Stück für Stück von dem Ereignis des Essens betroffen, so dass jedem Teil des Apfels ein Teilereignis von Essen entspricht und mit Abschluss des Ereignisses der letzte Teil des Objektreferenten von dem Ereignis erfasst ist (vgl. 
Krifka 1989: 159-161). Diese für Accomplishments typische Abarbeitung eines inkrementellen Patiens ${ }^{109}$ ist nun gerade der Grund dafür, dass machen-FVG mit Accomplishments nicht möglich sind. Machen hat in FVG, wie oben dargelegt, eine unterspezifizierte Bedeutung DO (x, y). Accomplishments sind von ihrer Ereignisstruktur demgegenüber verhältnismäßig reichhaltig, da sie, wie angedeutet, die Abarbeitung eines inkrementellen Patiens involvieren. Da machen in seiner Kernbedeutung DO (x, y) selbst kein Accomplishment ist, liegt auch keine semantische Übereinstimmung zwischen Nominalisierung und FV vor; folglich kann es auch keine Accomplishments als Nomina eines FVG selektieren.

\subsection{FVG mit haben}

\subsubsection{Der Bestand der Nomina}

Bereits zu Beginn dieses Kapitels wurde darauf hingewiesen, dass die FVG mit haben insofern ein besonderes Problem darstellen, als sie auf der einen Seite eine klare Präferenz für Nomina zeigen, die mentale Zustände, besonders Emotionen, bezeichnen, sie aber auf der anderen Seite mit einer größeren Zahl von Nomina genau dieser semantischen Klasse nicht kompatibel sind, vgl. (92) gegenüber (93).

(92) die Absicht, Achtung (vor), eine Ahnung, Angst, Anwandlung, Befürchtung, Begabung, Beobachtungsgabe, das Bestreben, Bedenken, ein Bewusstsein (von), Durst, Freude, Furcht, Gefallen, das/ein Gefühl, Gewissheit, Eigenschaft, Einstellung, Empfindung, Erkenntnis, Erwartung, Gespür, den Glauben, Hass, Hoffnung, Hunger, Interesse, Kenntnis, Kummer, Langeweile, Meinung, Misstrauen, Mühe, Mut, Panik, Plan, Respekt, Skrupel, Skepsis, Spaß, Traum, Überzeugung, Verdacht, Vermutung, Verständnis, Vertrauen, Vorstellung, Wahn, Wunsch, Zutrauen, Zweifel

(93) *Bestürzung haben, ${ }^{\star}$ Erschrecken haben ${ }^{\star}$ Traurigkeit haben, *Verzweiflung haben, ${ }^{\star}$ Liebe haben, ${ }^{\star}$ Verwunderung haben

Da sich auch in den bisher behandelten Fällen ein Blick auf die Bedeutung des FV als hilfreich für die Erklärung der Selektionseigenschaften von FVG erweisen

109 Croft (2012: 62f.) setzt neben dem klassischen inkrementellen Accomplishment allerdings auch noch eine Untergruppe „nonincremental accomplishment“ an, vgl. auch Rothstein (2001). 
hat, sei im Folgenden zunächst die Bedeutung von haben umrissen. Dabei handelt es sich freilich um einen ausgesprochen schwierigen Fall, der hier dementsprechend deutlich mehr Raum beansprucht als die bisher gebotenen Verbbeschreibungen.

\subsubsection{Die Bedeutung von haben}

\subsubsection{Haben: Polysemie und Unterspezifikation}

Das Spektrum der Bedeutungen und Funktionen, die haben einnehmen kann, ist außerordentlich vielfältig. Haben tritt als Auxiliar der Perfektbildung in Erscheinung, es weist in Verbindung mit $z u+$ Infinitiv deontische Bedeutung auf $(D u$ hast das zu tun!), und es kann schließlich zur Versprachlichung zahlreicher Relationen zwischen Subjekt und Objekt verwendet werden, wie die Beispiele in (94) zeigen.

(94) a. Jan hat ein Auto.

b. Jan hat eine Tante.

c. Jan hat einen Pickel auf der Stirn.

d. Jan hat große Angst.

e. Jan hatte gestern einen Fahrradunfall.

Nach Ausweis dieser Beispiele kodiert haben somit mindestens die Lesarten in (95), die man vorläufig wie folgt benennen kann: ${ }^{110}$

(95) a. alienables Besitzverhältnis ${ }^{111}$

b. inalienables Besitzverhältnis ${ }^{112}$

c. Lokativ (Verortung)

110 Für das englische have vgl. auch die (etwas umfangreichere) Lesartenzusammenstellung in Ritter/Rosen (1997: 296).

111 Zur Definition von Alienabilität/Inalienabilität vgl. Seiler (1983: 2); Heine (1997: 10); Stassen (2001: 954). Zur schwierigen Bestimmung des Konzepts ,Besitz‘ s. u.

112 Verwandtschaftliche Beziehungen, Körperteile, Teil-Ganzes-Relationen (BAUM-AST) werden in der Forschung traditionellerweise unter dem Begriff ,inalienabler Besitz“ (,inalienable possession“) zusammengefasst; zur Problematik dieser Klassifikation vgl. die Ausführungen von Heine (1997: 10-16). 


\section{d. Emotion \\ e. Ereignisinvolvierung}

Aufgrund der Heterogenität der Relationen, die haben ausdrücken kann, ist es der Forschung bisher schwer gefallen, einen gemeinsamen Nenner für haben zu finden. So wird bereits im ${ }^{1}$ DWB von den ,vielfach schillernden bedeutungen dieses wortes“ ('DWB 4, II, 50) gesprochen, und auch in der jüngeren Forschungsliteratur gilt haben (bzw. seine jeweilige lexikalische Entsprechung in einer anderen Sprache) als Verb, das sich einer präzisen semantischen Bestimmung weitgehend entzieht und notorisch vage bleibt - daher die bereits zum Topos gewordenen Charakterisierungen von haben als „colorless“ (Buck 1949: 740), „unspecific“, „multiply ambiguous“ (Seiler 1983: 64f.), „minimally meaningful“ (Bach 1967: 484) und sogar als „almost pathological“ (Bach 1967: 479) bzw. als „unsuitable lexical item“ (Bickerton 1990: 57). Auch Bierwisch (1983: 91) geht davon aus, dass die einzige Funktion von haben darin besteht, eine Verbindung zwischen zwei Argumenten zu etablieren, die grundsätzlich beliebig ist. Insofern liegt Bierwisch zufolge ein Fall extremer Unterspezifikation vor, der innerhalb der Verbsemantik eine Ausnahme darstellt (vgl. Lüdeling 1995: 1f). ${ }^{113}$

Zwar bietet sich die Annahme an, dass Verwendungen wie die in (95a), die alienablen Besitz zum Inhalt haben, gegenüber den anderen Verwendungen primär sind, da das Besitzen von Gegenständen ein kognitiv zentrales, früh gelerntes und in der Alltagserfahrung fest verankertes Konzept darstellt (vgl. Nikiforidou 1991: 198). Die Versprachlichung eines alienablen Besitzverhältnisses wäre demnach als der Prototyp innerhalb des semantischen Spektrums von haben anzusehen, dem gegenüber die anderen Funktionen als peripher bzw. abgeleitet zu gelten hätten (als primäres Ausdrucksmittel für alienablen Besitz wird haben/have übrigens auch bei Chomsky 1972: 201 angesehen).

Will man in dem Ausdruck alienablen Besitzes die prototypische Funktion von haben sehen, stellt sich jedoch umso dringlicher die Frage, wie das Konzept BESITZ überhaupt begrifflich zu fassen ist. Einen Versuch hierzu hat Taylor (1996: 340) vorgelegt. Dieser beschreibt alienablen Besitz als Konstellation der folgenden Merkmale:

113 Vorläufer dieser radikalen Unterspezifikationsthese ist Meillet (1923: 9): „Le plus souvent, le fr. avoir n'indique rien qu'un rapport entre le sujet et l'objet consideré“; „cette relation n'est pas seulement celle de possession; elle est du type le plus général“. 
(96) a. Besitzer ist ein Mensch

b. Besitz ist unbelebt

c. Besitzer hat ausschließliche Kontrolle über Besitz

d. Besitz kommt durch eine Transaktion wie Erwerb, Erbe zustande

Setzt man die Besitzlesart als Prototyp der lexikalischen Kategorie haben an, ergeben sich allerdings nicht unerhebliche Schwierigkeiten. So bleibt ungeklärt, aufgrund welcher Kriterien unter den vielen semantisch stark divergierenden Lesarten von haben gerade die Besitzlesart als Prototyp zu identifizieren ist. Es ist nicht ersichtlich, weshalb nicht z. B. auch die Experiencerlesart in (95d) oder die Ereignislesart in (95e) die Prototypen sein könnten, da Gefühle und Ereignisse nicht minder als das Konzept Besitz - alltäglich sind und zumindest Gefühle bereits in einem frühen Stadium der Ontogenese vorhanden sind. Fasst man die Summe aller Lesarten von haben als ,radiale Kategorie‘ (zum Begriff Lakoff 1987: 91-96) auf, d. h. als eine Kategorie, die in einem Netzwerk um eine prototypische Lesart herum organisiert ist, müsste zudem genau zu zeigen sein, in welcher Weise die einzelnen Lesarten von der als zentral vorausgesetzten Besitzinterpretation her motiviert sind. Gerade diese von der Besitzlesart ausgehenden Motivationsbeziehungen sind aber kaum plausibel zu machen. So ist noch nicht einmal klar, in welcher Beziehung die Konzepte alienabler und inalienabler Besitz untereinander stehen. Für Verwandtschaftsbeziehungen wie in (95b), die vielfach als Fall von inalienablem Besitz behandelt werden (Nichols 1992: 121f.; Heine 1997: 10; Barker 2011: 1112), bleibt z. B. offen, inwiefern hier überhaupt von Besitz gesprochen werden kann: In der Merkmalliste für alienablen Besitz, die Taylor zusammengestellt hat, ist für das Konzept TANTE HABEN nur ein einziges Merkmal einschlägig, nämlich die Eigenschaft ,Besitzer ist ein Mensch‘. Da ,x ist ein Mensch` ein sehr unspezifisches Merkmal ist, kann dies keine Zugehörigkeit zur Kategorie alienabler Besitz begründen.

An der vermeintlichen Prototypikalität der Besitzlesart von haben sind jedoch auch aus anderen Gründen Zweifel angebracht. So hatte bereits Brinkmann (1959: 184) festgestellt: „Die selbstverständliche Handhabung des Verbs legt den Gedanken nahe, dass ,haben“ ein Besitzen meine und deshalb vor allem mit konkreten Gegenständen verbunden werde, die dem Menschen als ,Habe‘ zugesprochen werden“. Da haben laut Brinkmann in Texten allerdings eher selten den Besitz eines konkreten Gegenstandes bezeichne, folgert er: „Dieser Gedanke trügt“ (ebd.). Brinkmann kann überdies zeigen, dass sich das gegenwartssprachliche haben auch historisch betrachtet „nicht dadurch von dem der älteren Zeit“ unterscheidet, „daß haben heute entdinglicht wäre; man wird im Gegenteil sagen können, daß die Ausdehnung der ,haben'-Fügung auf den gegenständlichen Bereich 
erst allmählich erfolgt ist“ (ebd.). Je weiter man also in der Sprachgeschichte zurückblickt, umso weniger lässt sich Brinkmann zufolge haben eindeutig als Besitzverb klassifizieren.

Wenn haben jedoch nicht oder zumindest nicht in erster Linie als Besitzverb anzusehen ist, stellt sich die Frage, was den Bedeutungskern von haben ausmacht, umso nachdrücklicher. Dieser Frage dadurch auszuweichen, dass man haben wie Bach (1967: 484) oder Bierwisch (1983: 91) von vornherein als bedeutungslos beschreibt, geht sowohl für das Englische als auch für das Deutsche zu weit: Immerhin sind, wie beispielsweise (98) und (99) zeigen, die Kombinationsmöglichkeiten von haben bzw. have nicht beliebig, wie für ein bedeutungsloses Verb zu erwarten wäre.

a. Hans hat Mut.

b. *Hans hat Feigheit.

a. I have a headache.

b. *I have hunger.

Angesichts der ,vielfach schillernden bedeutungen“ ('DWB 4, II, 50) ist die Bedeutung von haben aber in der Tat wohl nur als ein relativ stark unterspezifizierter semantischer Kern zu beschreiben. Worin dieser Kern von haben besteht, soll im Folgenden diskutiert werden.

\subsubsection{Lokalistische Erklärung}

Da haben semantisch außerordentlich schwer zu greifen ist, wird es in der Forschungsliteratur häufig als semantisch sekundär betrachtet und auf grundlegendere Konzepte zurückgeführt. ${ }^{114}$ So beschreibt Brugman (1988: 231) die Bedeutung von engl. have allgemein als „schema of interest or involvment“; andere sehen in der Kontrolle des Possessums durch den Possessor den semantischen Kern von haben (vgl. Hagège 1993: 93ff.; Langacker 1999: 162). ${ }^{115}$ Besonders verbreitet - und daher geradezu als „standard analysis“ (Jackendoff 1990: 261) zu

114 Dies hat diachron gesehen insofern eine gewisse Berechtigung, als Lexeme, die mit dt. haben vergleichbar sind, häufig etymologisch durchsichtig sind und daher verhältnismäßig junge semantische Entwicklungen darstellen (Buck 1949: 740).

115 Zur Problematik des Terminus „control“, der meist mit der Fähigkeit eines Possessors, das Possessum zu manipulieren, gleichgesetzt wird, vgl. Heine (1997: 3). Schwierig an dem Begriff „control“ ist Heine zufolge vor allem, dass bei einem unbelebten Possessor nur schwer von einer Manipulation des Possessums durch den Possessor die Rede sein kann. 
betrachten - ist die Hypothese, dass haben wie grundsätzlich alle Besitzausdrücke letztlich auf einer lokalen Relation basiert - ,all possession is location“ (Freeze 2001: 946; vgl. Lyons 1968: 388ff.; 1977: 722f.; Fillmore 1969: 15-18; Miller/Johnson-Laird 1976: 558; Clark 1978: 89; Jackendoff 1990: 261; Tuschinsky 2000: 123 u. a.). Wie diese Rückführung von haben auf eine räumliche Beziehung im Einzelnen modelliert wird, sei kurz an den verhältnismäßig gut ausgebauten Ansätzen von Jackendoff (1983; 1990) und Freeze (2001) illustriert.

\subsection{Die konzeptuelle Erklärung von Jackendoff (1983; 1990)}

Jackendoff steht für eine Herangehensweise an die lexikalische Semantik, die häufig als ,lokalistisch“ bezeichnet wird: „The basic insight of this [localistic, V. $\mathrm{H}$.] theory is that the formalism for encoding concepts of spatial location and motion, suitably abstracted, can be generalized to many other semantic fields“ (1990: 25; vgl. 1983: 188). ${ }^{116}$ Als Beleg für die elementare Funktion räumlicher Konzepte werden Bewegungsverben angeführt, die neben ihrer eigentlichen Bedeutung auch nicht-lokale Bedeutungen tragen; vgl. hier nochmals Jackendoffs in Abschnitt 5.2.5.1 bereits zitiertes Beispiel engl. go:

(99) a. The messenger went from Paris to Istanbul. (Lokation/Bewegung)

b. The inheritance finally went to Fred. (Besitz)

c. The light went from green to red. (Eigenschaftszuschreibung)

Das Konzept Go stellt Jackendoff zufolge ein wahrscheinlich angeborenes semantisches Primitiv dar, das zusammen mit anderen Primitiven wie PLACE, STAY, BE, MOVE, CAUSE das Gerüst der menschlichen Konzeptorganisation bildet. So wie das abstrakte Konzept GO in (99) sich in verschiedenen ,semantischen Feldern ${ }^{617}$ manifestiert - darunter auch im Feld ,Besitz ‘ -, so können auch die anderen konzeptuellen Primitive spezifische Feldausprägungen erhalten. Tritt das Primitiv BE (AT) - im Bestand der Primitive „the most basic unit“ (Jackendoff 2002: 360) - im Feld Besitz auf, wird es mit einem „field feature“ Possession versehen, das die Art

116 Jackendoff betont allerdings vor allem in jüngeren Arbeiten (z. B. Jackendoff 2002: 367), dass die grundlegende Rolle räumlicher Konzepte zu relativieren sei: Räumliche Konzepte bilden demzufolge nicht die Basis, von der die abstrakten Konzepte per Übertragung abgeleitet seien; vielmehr seien die semantischen Felder einschließlich Lokalisation „parallel instantiations of a more abstract schema“ (2002: 359).

117 Mit Wortfeldern der europäischen Tradition (dazu einführend Harm 2015: 90-94) hat dieses Konzept offenbar nicht unmittelbar etwas zu tun. Was mit ,Feldern` hier gemeint ist, bleibt im Wesentlichen unklar. 
der Argumente und die möglichen Inferenzen determiniert: „If the field feature on $\mathrm{BE}(\mathrm{x}, \mathrm{y})$ is Possession, $\mathrm{X}$ is an object and $\mathrm{Y}$ is a person who owns it; this configuration invokes inference rules about rights to use“ (2002: 360f.; vgl. 1990: 26f.). Mit Hilfe des für das Feld Possession spezifizierten Merkmals BE kann auch die Semantik von engl. have beschrieben werden. Dieses wird von Jackendoff wie folgt dekomponiert:

(100) Beth has the doll.

$$
\text { [state } \left.\left.\mathrm{BE}_{\text {Poss }}\left([\mathrm{DOLL}], \text { [place }_{\mathrm{AT}} \mathrm{T}_{\text {Poss }}([\mathrm{BETH}])\right]\right)\right]
$$

(Jackendoff 1983: 192)

Die Art und Weise, in der Jackendoff das Primitiv BE (AT) und das Feld Possession miteinander in Beziehungen setzt, ist jedoch nicht frei von einer gewissen Zirkularität, da seine Formulierung letztlich darauf hinausläuft, dass, wenn das Feldmerkmal ,Besitz' vorliegt, BE AT , besitzen` bedeutet. Zwar ist unbestritten, dass haben oftmals mit räumlicher Nähe einhergeht, dennoch kann Jackendoff außer dem ad hoc angesetzten Feldmerkmal ,Possession' kein notwendiges Kriterium anführen, das regelt, wann genau BE AT bloß als lokal und wann es als besitzanzeigendes Verb haben zu interpretieren ist. Denn dass nicht alle Relationen, die eine räumliche Nähe bezeichnen, mit haben versprachlicht werden können, liegt auf der Hand, vgl. (101).

(101) a. Die Garage des Nachbarn ist am/beim Haus.

b. ${ }^{\star}$ Das Haus hat die Garage des Nachbarn.

Räumliche Nähe ist somit kein hinreichendes Kriterium für die Definition des Konzepts ,haben` (vgl. auch Heine 1997: 3; Jacob 2003: 36). Die unmittelbare räumliche Nachbarschaft zwischen zwei Objekten ist, auch wenn sie der kanonische Fall sein mag, noch nicht einmal notwendig für haben, wie die Vereinbarkeit der haben-Äußerung in (102a) mit (102b) zeigt.

(102) a. Das Haus hat eine Garage.

b. Die Garage befindet sich zwei Blocks weiter.

Eine angemessene Beschreibung der Bedeutung von haben ist mit Jackendoffs Dekomposition ,BE AT‘ somit noch nicht erreicht. 


\subsection{Die syntaktische Erklärung von Freeze (1992)}

Eine radikal lokalistische Erklärung von Possessiva im Allgemeinen und von haben im Besonderen hat auch Freeze (1992; 2001) vorgelegt. Anders als Jackendoffs konzeptuelle Deutung ist die Hypothese von Freeze jedoch durchweg syntaktisch ausgerichtet. Ein wesentlicher Unterschied gegenüber Jackendoffs Herleitung

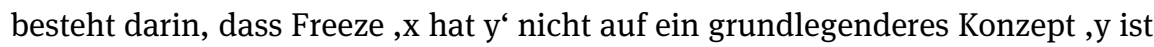

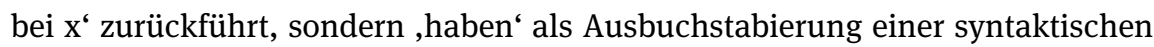
Struktur versteht. Da die Hypothese von Freeze zumindest innerhalb der generativen Tradition als sehr einflussreich gelten kann, ${ }^{118}$ ist ein Blick auf diesen Ansatz in jedem Fall lohnend.

Die Grundthese „all possession is location“(Freeze 2001: 946) kann zunächst an folgendem Gegensatzpaar aus dem Russischen deutlich gemacht werden:

$$
\begin{aligned}
& \text { a. na stole byla kniga } \\
& \text { auf TischGEN. COP.3.SG.PRÄT. Buch } \\
& \text {,Auf dem Tisch war ein Buch.` } \\
& \text { b. u menja byla kniga. } \\
& \text { bei 1.SG.GEN. COP.3.SG.PRÄT. Buch } \\
& \text {,Ich hatte ein Buch.` }
\end{aligned}
$$

Da zwischen dem lokativischen Ausdruck in (103a) und dem possessiven Ausdruck in (103b) eine erkennbare Analogie besteht, stellt Freeze die Hypothese auf, dass die possessive Struktur aus der lokalen Struktur abgeleitet ist. Das Possessivum sei nichts anderes als ein „existential with a [+human] location“ (Freeze 2001: 946). Entscheidend für das Zustandekommen der possessiven Interpretation der lokalen Grundstruktur ist die Bewegung des „[+human] location argument“ (ebd.) in die Spezifiziererposition der IP, vgl. (104).

118 Vgl. u. a. die Rezeption bei Kayne (1993: 6f.); den Dikken (1997: 132f.), Ritter/Rosen (1997: 297), Harley (2002: 48-51), Larson/Cho (2003: 227-229), Lasnik/Uriagereka (2005: 78); Businger (2011). 
(104)

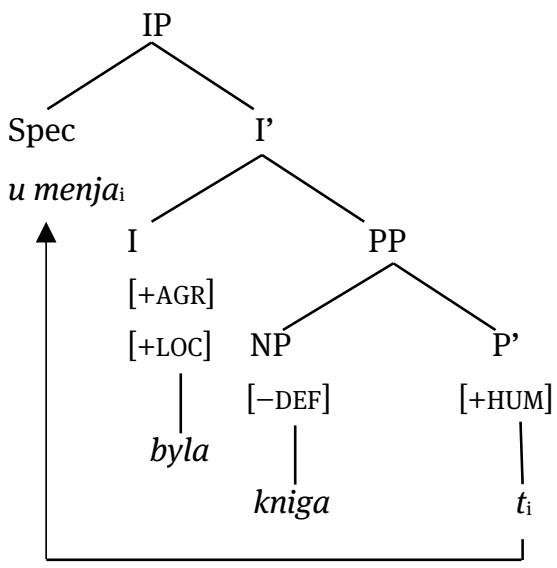

(vgl. Freeze 2001: 947)

(105) a. o menino está com fome

Das Kind ist mit Hunger

,Das Kind ist hungrig.

b. o menino tem fome

Das Kind hat Hunger

,Das Kind ist hungrig.

Im Ausdruck (105a), der eine Verbindung von Kopula und com enthält, ${ }^{119}$ liege, so Freeze, die lokative Basisstruktur vor, während (105b) als davon abgeleitet aufzufassen sei. Diese Ableitung wird in den folgenden drei Stufen modelliert: (i) Im Gegensatz zu der für das russische Beispiel (103) postulierten Bewegung des „location argument “ $P$ ' nach [Spec, IP] werde in diesem Fall nicht P' als Ganzes, sondern nur die von P' dominierte NP mit dem Merkmal [+hum] in die Spezifiziererposition angehoben; die Präposition werde dabei zunächst zurückgelassen. (ii) In einem weiteren Schritt werde die Präposition dann in den INFL-Knoten bewegt, wo sie mit diesem per Kopf-an-Kopf-Adjunktion zu „I.P“ inkorporiert werde; vgl. (106):

119 Auf den Unterschied zwischen estar und ser, die im Portugiesischen (wie im Spanischen) beide als Kopula vorkomen, geht Freeze nicht ein. 
(106)

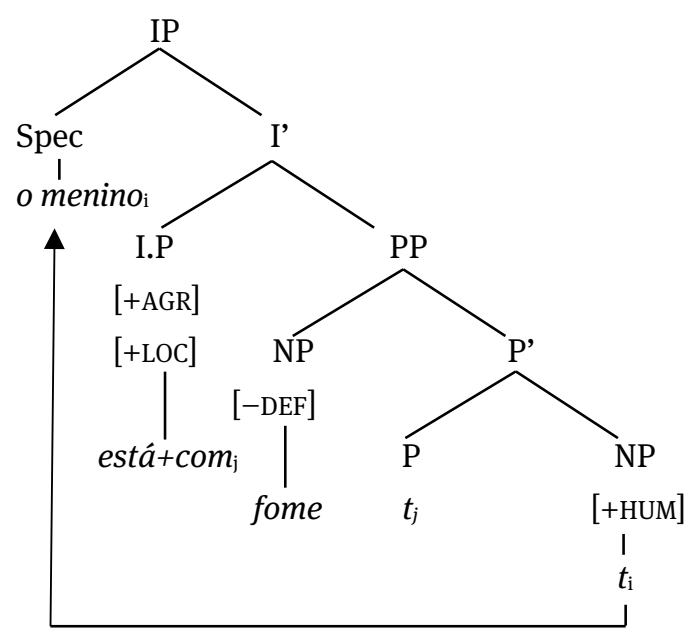

(Freeze 2001: 950)

(iii) Der letzte Schritt der Herleitung bestehe darin, dass die so entstandene Konstituente Kopula + P (está com) als ein Lexem realisiert werde. Port. ter ,haben“ ist Freeze zufolge somit nichts anderes als die lexikalische Ausbuchstabierung eines zugrunde liegenden estar com: ,, have“ can be conceived as the spellout of the set of inflectional features of a P-augmented ,be“ “ (Freeze 1992: 588). Was Freeze hier am Beispiel des Portugiesischen demonstriert, soll wohlgemerkt jedoch nicht nur für ter, sondern grundsätzlich für ,haben'-Lexeme aller Sprachen gelten(, ,have‘ ist always a derived form“, Freeze 2001: 951). ,Haben“ ist somit letztlich kein aus dem Lexikon projiziertes Verb, sondern die phonetische Realisierung eines funktionalen Kopfes, der mit einer Präposition verschmolzen ist. ${ }^{120}$

Die von Freeze aufgestellte Hypothese hat den Vorteil, dass port. ter bzw. die ,haben'-Ausdrücke anderer Sprachen auf einen Nenner mit anderen Possessivausdrücken gebracht werden können, die eine Kopula enthalten (wie z. B. mit dem russischen Ausdruck in [103]). Da possessive Ausdrücke mit einer ,haben‘-

120 Dass Freeze in dieser Ableitung das Possessum in der Spezifiziererposition der PP generiert, mag zunächst überraschend erscheinen. Eigentlich würde man erwarten, dass in der Spezifiziererposition einer PP typischerweise Elemente wie mitten in Paris stehen. Der von Freeze angesetzten lokativen PP wird hier allerdings insofern ein besonderer Status zugesprochen, als sie als „,complete functional complex (CFC)“ in Anlehnung an Chomsky (1986: 15) beschrieben wird. Auf alle PPs, so stellt Freeze (1992: 559) ausdrücklich fest, könne diese Analyse jedoch nicht ausgedehnt werden. 
Lexikalisierung zumindest nach verbreiteter Auffassung seltener sind als die lokativisch formulierte Possession - Bach (1967: 479) hatte ,haben' aufgrund seiner Seltenheit als „almost pathological“ bezeichnet ${ }^{121}$-, ist es grundcsätzlich auch plausibel, dass die seltenere Erscheinung auf die häufigere zurückgeführt wird.

Die von Freeze präsentierte Deutung von ,haben' bringt jedoch auch Probleme mit sich, auf die im Folgenden eingegangen werden soll. ${ }^{122}$ So ist zunächst anzumerken, dass die Alternation von port. estar com vs. ter, auf die Freeze sich als einzigen Beleg für Herleitung von ,haben'-Ausdrücken stützt, nur in einer relativ eingeschränkten semantischen Domäne auftritt, nämlich beim Ausdruck von Empfindungen, vgl. dazu auch die folgenden Beispiele:

(107) a. estar com calor vs. ter calor ,schwitzen“

b. estar com frio vs. ter frio ,frieren“

c. estar com sono vs. ter sono ,müde sein“

d. estar com dores de cabeça vs. ter dores de cabeça ,Kopfschmerzen haben'

e. estar com medo vs. ter com medo,Angst haben“

(s. Dicionario da lingua portuguesa contemporânea 2, 3545)

Gerade bei alienablem Besitz besteht jedoch kein derartiges Nebeneinander; die Variante estar com ist hier nicht zulässig, vgl. (108).

(108) a. Ele tinha uma casa perto do lago.

Sie hatte ein Haus nah P+DET See

b. ^Ele está com uma casa perto do lago.

Wenn, der Annahme von Freeze folgend, sämtliche prädikativen Possessivausdrücke einschließlich ,haben“ von einem lokativen Grundmuster abgeleitet sind, muss als erstaunlich gelten, dass im Portugiesischen gerade bei den Possessiva

121 Zur Auffassung von Bach (1967) und Freeze (1992, 2002), dass nur wenige Sprachen der Welt einen verbalen Possessionsausdruck besitzen, der mit dt. haben vergleichbar ist, und die meisten Sprachen verbale Possession stattdessen mit einer lokativischen Kopula-Konstruktion verbalisieren, vgl. u. a. Heine (1997: 6) sowie Stassen (2001: 955). Hierbei fällt allerdings auf, dass die kaukasischen Sprachen, in denen ,haben'-Ausdrücke sehr verbreitet sind (vgl. Boeder 1980), nicht genannt werden.

122 Eine Betrachtung weiterer syntaktischer Details kann hier nicht erfolgen. Dazu sei auf Harley (2002: 49-51) verwiesen. 
im engeren Sinne die Alternation estar com vs. ter nicht vorliegt, während sie bei Ausdrücken für Empfindungen, die im Hinblick auf Possession eher als peripher einzustufen sind, möglich ist. Das Portugiesische bietet somit keine guten Argumente für die Rückführung der ,haben'-Ausdrücke aller Sprachen auf eine zugrundliegende Inkorporation von Kopula und Präposition. ${ }^{123}$

Ein damit zusammenhängendes Problem der Herleitung von Freeze tritt zu Tage, wenn man Sprachen in Betracht zieht, die über zwei (oder mehrere) ,haben'-Ausdrücke verfügen. So stehen im Gotischen die beiden Verben haban und aigan einander gegenüber: haban drückt zumeist alienablen Besitz aus und bezieht sich auf Konkreta in der Rolle des Possessums, aigan hingegen steht grundsätzlich für inalienable Besitzverhältnisse und ist überwiegend mit Abstrakta kombiniert (dazu Häusler 2004: 136-139). ${ }^{124}$ Wären die Verben des Gotischen Ausbuchstabierungen einer zugrundeliegenden Inkorporation von Kopula und Präposition, wie Freeze unterstellt, müssten Regeln postuliert werden, die diese Ausbuchstabierungen mit den semantischen Merkmalen des jeweiligen Possessums (hier Konkretum vs. Abstraktum) abgleichen. Ähnliches gilt übrigens auch für ter und estar com im Portugiesischen: Auch hier müsste eine Regel aufgestellt werden, die sicherstellt, dass zugrundeliegendes estar com bei einem Possesum, das keinen mentalen Zustand bezeichnet, obligatorisch als ter realisiert werden muss, während die Lexikalisierung als ter bei Objekten wie in (108) optional ist. Zusatzregeln dieser Art sind jedoch überflüssig, wenn man ter bzw. estar com schlicht als lexikalische Einheiten behandelt. Dass Verben semantische Selektionsbeschränkungen hinsichtlich ihrer Argumente aufweisen, ist völlig erwartbar und unproblematisch, wenn man sie nicht als Realisierungen funktionaler Köpfe, sondern als lexikalische Köpfe ansieht (vgl. als Beispiele für Selektionsrestriktionen die Verben dt. töten vs. erlegen, schießen (transitiv) vs. erschießen oder putzen vs. waschen mit ihren unterschiedlichen semantischen Subkategorisierungen).

123 Wie (106) deutlich macht, analysiert Freeze die Kopula ,be‘ als Realisierung des Infl-Kopfes (dies in grundsätzlicher Übereinstimmung mit einem Großteil auch der späteren generativen Literatur, welche die Kopula als Spellout eines funktionalen Kopfes betrachtet, vgl. die Übersicht in Maienborn 2003: 23). Wenn nun ,be‘ die Ausbuchstabierung von Infl $^{0}$ ist, wäre ,have' gewissermaßen die Ausbuchstabierung der Ausbuchstabierung. Dies ist wenig einleuchtend.

124 Auch im Georgischen gibt es zwei Verben, die als Entsprechungen von dt. haben angesehen werden können: mqavs und makvs, wobei mqavs mit belebtem, makvs mit unbelebtem Possessum steht (vgl. Boeder 1980: 208f.); ,belebt' schließt dabei neben Menschen und Tieren auch Fahrzeuge ein (persönliche Mitteilung M. Job). 
Unter der Annahme, dass haben eine lexikalische Einheit darstellt, lässt sich ein weiteres Problem von Freezes Hypothese - die Polysemie von ,haben'-Ausdrücken - relativ leicht beheben. Wird ,haben` als Realisierung syntaktischer Gegebenheiten angesehen, wie Freeze annimmt, müsste auch jede einzelne Bedeutungsvariante innerhalb des Gebrauchsspektrums von ,haben' als Derivat unterschiedlicher syntaktischer Strukturen beschrieben werden. In diese Richtung geht z. B. der in der Tradition von Freeze stehende Versuch von Ritter/Rosen: „[...] the various interpretations of have are derived from the syntactic structure“ (1997: 295). Eine solche Erklärung belastet die Syntax allerdings außerordentlich stark, und es stellt sich zudem die Frage, warum dann nicht viel mehr Verben mit semantischer Variation syntaktisch erklärt werden müssen, wie Lasnik/Uriagereka (2005: 78) in diesem Zusammenhang zu Recht anmerken: „[...] why don't all verbs find the expression of whatever it is they mean through some sort of grammatical construction?“. Im Lexikon dagegen wäre eine ausgefaltete Polysemie, wie ,haben'-Ausdrücke sie in der Regel zeigen, nicht überraschend und verhältnismäßig gut beschreibbar.

Für den Ansatz einer Lexikoneinheit ,haben‘ und gegen die von Freeze vertretene Herleitung sprechen nicht zuletzt die syntaktischen Probleme, die seine in der Government-and-Binding-Tradition formulierte Analyse aufweist. Diese betreffen im Wesentlichen die Kasuszuweisung. Im Normalfall ist davon auszugehen, dass die Präposition com der NP o menino in der D-Struktur, d. h. vor Einsetzen der Bewegungen, einen lexikalischen Kasus zuweist (vgl. Haegemann 1991: 314f.). ${ }^{125}$ Wenn nun o menino in [Spez, IP] bewegt wird, bleibt offen, wie mit dem obliquen Kasus, den o menino aus der D-Struktur mitbringen müsste, zu verfahren ist - mit einem obliquen Kasus in der Subjektposition [Spec, IP] läge auf jeden Fall eine ungrammatische Struktur vor. In Bezug auf das Portugiesische, das kaum morphologische Kasusmarkierung kennt, mag das Problem auf den ersten Blick als vernachlässigbar gelten. Dennoch treten auch hier im Pronominalbereich morphologisch distinkte Formen auf, vgl. etwa eu ,ich' und tu ,du', welche nach der Präposition com als -igo bzw. -tigo realisiert werden. Gerade die kontrahierten Pronominalformen port. comigo, comtigo, comsigo ,mit sich', conosco ,mit uns', convosco ,mit euch' zeigen umso deutlicher, dass eine Bewegung des Pronomens aus einer com regierten PP heraus wenig plausibel ist. Bei Sprachen, die ein ausgebautes System morphologischer Kasus besitzen, tritt das Problem noch schärfer in Erscheinung. Wollte man etwa dt. haben im Sinne von

125 Chomsky (1981: 170) spricht von „,inherent case“. In Anlehnung an den in der deutschsprachigen Literatur verbreiteten Usus (dazu Dürscheid 1999: 52) sei hier stattdessen von „lexikalischem Kasus“ die Rede. 
Freeze erklären, so läge auch hier die Schwierigkeit vor, dass eine NP aus einer PP (z. B. mit bei als Kopf) herauszubewegen wäre. Weil diese bereits in der DStruktur einen lexikalischen Kasus erhalten hat, in diesem Fall den Dativ, ergäbe die Anhebung in die [Spec, IP]-Position eine ungrammatische Äußerung. ${ }^{26}$

Da sowohl Jackendoffs konzeptuelle Dekomposition von haben als auch die syntaktisch motivierte lokalistische Analyse, die Freeze vorgelegt hat, Schwächen aufweisen, ${ }^{127}$ erscheint es angezeigt, nach nicht-lokalistischen Erklärungsalternativen zu suchen. Eine solche Analyse bietet das kognitivistische Modell von Langacker (1993; 1999).

\subsubsection{Haben als „reference point construction“ (Langacker 1993; 1999)}

In kognitivistischen Ansätzen wird haben nicht als bloße Ausbuchstabierung syntaktischer Strukturelemente, sondern als bedeutungstragende lexikalische Einheit behandelt. Freilich ist auch hier die Bedeutung, die haben zugeschrieben wird, sehr abstrakt. So identifiziert Langacker (1993; 1999) eine sehr allgemeine „reference point function“ als semantischen Kern des Verbs („[...] the reference point function of have, which I take to be its only constant import“1999: 183). Ein ,reference point“ ist Langacker zufolge ein Konzept, das zur Situierung eines anderen Konzepts gebraucht wird (analog zu einem Referenzpunkt in der Geometrie). Das Konzept, das mit Hilfe eines Referenzpunktes situiert wird, stellt das ,target‘ dar. Die Menge der potentiellen targets, die auf einen Referenzpunkt bezogen werden können, bilden dessen sog. ,dominion“ (,the conceptual domain (or set of entities) to which a particular reference point affords direct access“, Langacker 1993: 6). Die Bedeutung von haben (bzw. engl. have) ist nun, dass es im Subjekt einen Referenzpunkt (R) für das ein im Objekt versprachlichtes Target (T) bereitstellt:

(109) He (R) has a lot of freckles (T).

126 Die Annahme einer latenten, semantisch leeren Präposition, die dann nur ein funktionaler Kopf wäre, bliebe eine ad-hoc-Annahme, die das Problem der Kasuszuweisung nicht zufriedenstellend löst.

127 Auch die Spracherwerbsforschung bietet keine verlässlichen Anhaltspunkte für die Klärung der Frage, ob Possession primär ist oder die spezifische Ausprägung lokaler Relationen darstellt; für ein Referat der Positionen s. Barker (1995: 44-50). Es scheint sich auf jeden Fall so zu verhalten, dass Kinder bereits sehr früh über einen Basisbegriff ,Besitz‘ verfügen, auch wenn dieser nicht immer klar von räumlichen Relationen getrennt werden kann. 
Die Funktion, eine Relation zwischen einem Referenzpunkt und einem Target herzustellen, gilt Langacker nicht nur für engl. have, sondern generell für possessive Ausdrücke (Genitivattribute, Possessivpronomina). Bei have tritt neben die grundlegende Referenzpunkt-Funktion, die in allen Verwendungen des Verbs zu greifen ist, optional das Bedeutungsmerkmal ,physical control‘ hinzu: „[...] have also displays a variety of more specific senses in which some vestigial notion of control or access is overlaid on this schematic value“ (1999: 183). Das - von Langacker nicht näher bestimmte - Merkmal ,physical control‘ ist vor allem für die Besitzlesart (he has a car etc.) relevant.

Problematisch an Langackers Bestimmung der Semantik von have ist vor allem, dass sie zu allgemein bleibt. Sie unterscheidet nicht ausreichend zwischen einem wie auch immer verstandenen übergreifenden Begriff von ,Possession“/,Possessiv‘, unter den laut Langacker (1999: 73) auch Genitivattribute, Pronomina und PPs mit engl. of fallen, und der spezifischen Semantik von haben. Wäre die Herstellung einer Relation zwischen Referenzpunkt und Target das Kernmerkmal sämtlicher ,possessiver‘ Ausdrücke einschließlich engl. have/dt. haben, so könnte nicht erklärt werden, weshalb z. B. die Referenzpunkt-TargetRelation im Englischen durch eine Genitiv- bzw. of-Konstruktion ausgedrückt werden kann und gleichzeitig eine entsprechende have-Konstruktion nicht möglich ist:

(110) a. The destruction (T) of the city (R)/the city's (R) destruction (T)

b. *The city (R) has destruction (T).

Präzise Vorhersagen, welche Relationen mit have versprachlicht werden können und welche nicht, leistet Langackers Ansatz somit nicht. ${ }^{128}$

\subsubsection{Haben als Indizierungsrelation}

Langacker hatte im Subjekt von have/haben den Referenzpunkt, im Objekt das Ziel/,Target‘ dieser Referenz gesehen. Taylor merkt dazu allerdings kritisch an,

128 Aufgrund dieser Unklarheit hat Taylor (1996: 236-264) versucht, Langackers PossessionsBegriff zu präzisieren, in dem er das Konzept der „cue validity“ bzw. ,informativity“ des Possessors einführt. Auf Näheres sei an dieser Stelle nicht eingegangen, da Taylor über Possession allgemein spricht und sich zu engl. have in keiner Weise äußert. 
dass die von Langacker angenommene Richtung der Verweisrelation Referenzpunkt $\rightarrow$ Target wenig plausibel ist:

[...] a speaker, in producing a possessive, manifestly does not start out from reference point $\mathrm{R}$, only then to select a target from the set of entities that might be identified from R. Rather obviously, the speaker starts with the intention of referring to a target entity $\mathrm{T}$, and then selects the reference point most appropriate to this purpose.

(Taylor 1996: 237; ähnlich Barker 2011: 1125) ${ }^{129}$

Für haben bedeutet dies, dass auch hier eher eine vom Objekt (Langackers ,Target') auf das Subjekt (Langackers ,Referenzpunkt') gerichtete Relation anzunehmen ist. In Bezug auf engl. have verfolgt Taylor diesen Gedanken allerdings nicht weiter. Im Folgenden soll aber genau an diese Beobachtung anschließend dargelegt werden, dass die Bedeutung von haben in der Tat im Wesentlichen darin besteht, eine Indizierungsrelation zwischen Objekt-NP und Subjekt-NP herzustellen, und zwar insofern, als das durch die Objekt-NP kodierte Konzept ein Index für das durch das Subjekt versprachlichte Konzept darstellt.

Bevor die Hypothese, die Semantik von haben bestehe im Wesentlichen in der Etablierung einer Indizierungsrelation, ausgefaltet werden kann, sind grundlegende Eigenschaften von Indexikalität zu beschreiben. Es sei angenommen, dass ein Konzept $\mathrm{X}$ ein Konzept $\mathrm{Y}$ indiziert, wenn die folgenden Bedingungen gegeben sind:

(i) Folgerungsrelation: Vom Vorhandensein des Konzepts X kann auf das Vorhandensein des Konzepts Y geschlossen werden (von Rauch auf Feuer, von Fußspur auf Fuß), nicht notwendigerweise umgekehrt (nicht jedes Feuer produziert Rauch, nicht jeder Fuß hinterlässt eine Spur).

(ii) Unterscheidbarkeit: Die involvierten Konzepte dürfen keine identische Extension aufweisen, sondern müssen als distinkte Entitäten wahrnehmbar sein. (Die Fußspur muss von Fuß unterschieden werden können, der Rauch muss getrennt vom Feuer wahrnehmbar sein, damit eine Indexfunktion etabliert werden kann.)

(iii) Kontiguität in Zeit und Raum: Die involvierten Konzepte müssen in Zeit und Raum aufeinander beziehbar sein (frische Fußspuren, die sichtbar zu einer

129 Im Sinne Langackers äußert sich auch Jacob (2003: 43): „Eine Funktion dieser Formen [der Possessiva, V. H.] ist es, durch den ,Possessor'-Ausdruck einen Referenten zu benennen, der die Basis für die Referenzierung eines anderen Nominalausdrucks (nämlich das ,Possessum`) darstellt“. 
Person hinführen, haben höhere Indexikalität als z. B. ein vereinzelter Fußabdruck im Wald).

(iv) Exklusivität der Relation: eine Fußspur indiziert eine Person X, nicht mehrere.

(v) Unmittelbarkeit (Intransitivität) der Relation: Fußspur ist Index für Person X, nicht z. B. für deren Verwandte.

Kriterium (i) ist dabei das wichtigste; die anderen Kriterien ergeben sich daraus. Zieht man Beispiele für die oben eingeführten Lesarten von haben heran, so zeigt sich, dass in der Tat eine Indizierungsrelation Objekt-NP $\rightarrow$ Subjekt-NP postuliert werden kann: Vom Gegebensein des in der Objekt-NP kodierten Sachverhalts bzw. Referenten kann auf das Gegebensein des Referenten in der Subjekt-NP geschlossen werden (Eigenschaft [i]). Wann immer ein Ereignis UNFALL gegeben ist, gibt es auch jemanden, der von diesem Ereignis betroffen ist, und wenn von einem SCHORNSTEIN die Rede ist, muss auch die Existenz eines zugehörigen GEBÄUDES angenommen werden.

(111) a. Peter hatte einen Unfall.

$\exists$ e[Unfall (e)] $\rightarrow \exists \mathrm{x}$ [Betroffener (x, e)]

b. Peter hat eine Tochter.

$\exists \mathrm{y}[\operatorname{Tochter}(\mathrm{y})] \rightarrow \exists \mathrm{x}[\operatorname{VATER}(\mathrm{x}, \mathrm{y})]$

c. Peter hat Pickel.

$\exists \mathrm{y}[\operatorname{PiCKEL}(\mathrm{y}) \rightarrow \exists \mathrm{x}[\operatorname{TRÄGER}(\mathrm{x}, \mathrm{y})]$

d. Das Haus hat einen Schornstein.

$\exists \mathrm{y}[\operatorname{SchORNSTEIN}(\mathrm{y})] \rightarrow \exists \mathrm{x}[\operatorname{GEBÄUdE}(\mathrm{x})]$

e. Das Auto hat einen Kratzer.

$\exists \mathrm{y}[\operatorname{Kratzer}(\mathrm{y})] \rightarrow \exists \mathrm{x}[\operatorname{Betroffener}(\mathrm{x}, \mathrm{y})]$

f. Das Wort Amour hat die Bedeutung ,Liebe‘.

$\exists \mathrm{y}[\operatorname{WoRTBEDEUTUNG}(\mathrm{y})] \rightarrow \exists \mathrm{x}[\operatorname{WoRT}(\mathrm{x})]$

Die in diesen Beispielen vorliegenden Objekt-NPs enthalten Konzepte, die relativ zu einem anderen Konzept bestimmt sind und die daher zumindest in der hier vorliegenden Lesart als relational gelten können (im Sinne von Löbner 1985: 292). Verbindungen von haben mit nicht-relationalen Konzepten wie ,Berg“ oder ,Baum` - nach Löbner (ebd.) werden diese Konzepte als ,sortal‘ bezeichnet - gibt es ebenfalls. Diese werden weiter unten diskutiert.

Wenn haben also als Konzept mit zwei Argumenten verstanden werden kann, die in einer Relation „INDEX“ stehen, so muss freilich einschränkend hinzugefügt 
werden, dass nicht jede Indexikalitätsrelation durch haben ausgedrückt wird. So besteht für das indexikalische Verhältnis zwischen den Konzepten FEUER und RAUCH nicht die Option einer Versprachlichung durch haben. Gleiches gilt für ein weiteres klassisches Beispiel für Indexikalität, nämlich FussSPUR und Fuss:

(112) a. 'Das Feuer hat Rauch.

b. 'Der Fuß hat eine Spur im Wald.

Der Grund für die fragliche Akzeptabilität von haben in (112) ist darin zu sehen, dass es auf Zustandsrelationen eingeschränkt ist (vgl. auch Benveniste 1966: 198; Lyons 1968: 395 zu den ,haben'-Wörtern unterschiedlicher Sprachen). Eine Relation zwischen zwei prozesshaften Referenten wie FEUER und RAUCH kommt daher nicht in Betracht. Auch der Referent von SPUR ist nicht als ,Zustand von Fuss' analysierbar. Die semantische Beschreibung von haben wird hier dementsprechend durch ein Bedeutungspostulat ergänzt, dass die Subkategorisierung als „Zustand" des Possessors festlegt.
a. $\forall \mathrm{x} \forall \mathrm{y}\left[\operatorname{HABEN}^{\prime}(\mathrm{x}, \mathrm{y}) \rightarrow \operatorname{INDEX}(\mathrm{y}, \mathrm{x})\right]$
b. $\forall \mathrm{x} \forall \mathrm{y}\left[\operatorname{HABEN}^{\prime}(\mathrm{x}, \mathrm{y}) \rightarrow\right.$ ZUSTAND $\left.(\mathrm{x})\right]$

Damit ist für haben eine relativ stark unterspezifizierte Bedeutung angesetzt. Im Unterschied zu Bierwisch (1983), der die Art der von haben ausgedrückten Relation vollständig offen gelassen hat, wird mit der Beschreibung in (113) gleichwohl kein semantisch leeres haben postuliert.

$\mathrm{Zu}$ klären ist freilich noch, wie sich die indexikalische Grundbedeutung des Verbs zur ,besitzen'-Lesart verhält. In dieser im engeren Sinne possessiven Interpretation steht haben im Normalfall mit nicht-relationalen (sortalen) Nomina:

\section{Anna hat ein Auto.}

Im Gegensatz zu relationalen Nomina verweisen sortale Nomina nicht per se auf ein anderes Konzept; sortalen Nomina ist daher auch keine Indexikalität, d. h. keine Verweisfunktion, inhärent. Durch das besitzanzeigende haben in (114) wird gleichwohl sekundär Relationalität bzw. Indexikalität hergestellt. Das Verb $h a-$ ben fungiert damit in gewisser Weise als „Type Shifter“: Dadurch, dass das sortale Nomen Auto als ,Besitz von X“ markiert wird, wird ihm Relationalität zugeschrieben; Auto trägt damit einen Index ,von Person X'. Die sortalen Eigenschaften des Referenten von Auto sind im Kontext der Äußerung (114) nicht 
von Interesse; hier steht lediglich die Relation des Referenten zu einer Person, seine Indexfunktion für diese Person im Fokus.

Indexikalität ist somit eine elementare Eigenschaft des Konzepts ,Besitz‘. Letzteres ist dabei sicher das reichhaltigere Konzept, da etwa noch die Eigenschaft ,Kontrolle des Besitzes durch den Besitzer` oder ,längere Dauer` hinzukommt (s. Taylor 1996: 340). Dass Indexikalität ein wesentlicher Bestandteil von ,Besitz' ist, zeigt sich auch daran, dass die oben aufgeführten Eigenschaften für Indexikalität ausnahmslos auch auf die Besitzrelation zutreffen:

(i) Folgerungsrelation: Possessum indiziert Possessor (s. o.).

(ii) Unterscheidbarkeit: Possessum und Possessor sind nicht referenzidentisch.

(iii) Kontiguität in Zeit und Raum: Possessum und Possessor befinden sich typischerweise in räumlicher Nähe (s. Taylor 1996: 340).

(iv) Exklusivität der Relation: Einem Possessum entspricht in der Regel nur ein Possessor; mein Auto gehört in der Regel nicht zugleich einem anderen. ${ }^{130}$ Umgekehrt entsprechen einem Possessor problemlos mehrere Possessa: mein Haus, mein Auto ...).

(v) Unmittelbarkeit (Intransitivität) der Relation: Ein Possessum ist nicht übertragbar; mein Auto ist nur meines und nicht das meines Vaters oder Großvaters.

Eine Herausforderung für jede Beschreibung der Semantik von haben ist die existentiale Verwendung des Verbs, wie sie in dem schweizerdeutschen bzw. südwestdeutschen Beispiel (115) vorliegt.

(115) In der Schweiz hat es viele Berge.

Diese existentiale Lesart wird bei Freeze (1992) und Partee (2004: 285) als grundlegend für alle Bedeutungsvarianten angesehen, die nicht im engeren Sinne possessiv sind. Die Ausdrücke für Existentialität sind allerdings in vielen Sprachen ganz offensichtlich von anderen Ausdrücken abgeleitet, wie die Fälle in (116) zeigen:

130 Das schließt nicht aus, dass im Einzelfall auch mehrere Personen eine Sache besitzen können (Ehepaar, Erbengemeinschaft, Wohngemeinschaft, Verein usw.). Gleichwohl liegt auch in diesen Fällen eine abstrakte, aus mehreren Personen gebildete Einheit vor, die exklusiv über das Possessum verfügt (Ehepaar $X$ und nicht Ehepaar $Y$ ). Auch juristisch wird Besitz als tatsächliche Gewalt einer Person über eine Sache definiert. Teil- und Mitbesitz stellen dem gegenüber Sonderfälle dar. 
(116) a. dt. es gibt, es besteht

b. schwed. det finns

c. lat. existo < ex-sisto,trete hervor"

d. agr. heurísketai ,es gibt, es findet sich“

Umgekehrt ist in einer Reihe von Sprachen ein Ausdruck für ,haben' klar als Quelle für ein Existential identifizierbar. Heine (1997: 95) nennt als Beispiele frz. il y a, span. hay (+ Ortsangabe), bulgarisch ima (,es hat' > ,es gibt'). Daher ist die Annahme, die existentiale Bedeutung stelle die Basis für alle oder die meisten Bedeutungen von haben dar, alles andere als zwingend. Setzt man dies voraus, wäre für die Standardsprache norddeutscher Prägung zudem eine Basislesart anzunehmen, die gar nicht overt vorkäme. Dies ist zwar nicht auszuschließen, erscheint aber wenig vorteilhaft, wenn es andere Erklärungsmöglichkeiten gibt.

Im Rahmen des hier vertretenen Ansatzes ist die existentiale Verwendung von haben somit sekundär. Sie wäre aus einer Eigenschaftszuschreibung wie in (117) herzuleiten.

(117) Die Schweiz hat viele Berge.

Hier liegt insofern Indexikalität vor, als die DP viele Berge auf einen Ort verweist, dessen Hauptcharakteristikum eben in einer markanten geologischen Eigenheit besteht. (117) wäre damit als ,Die Schweiz ist durch viele Berge gekennzeichnet zu paraphrasieren. Daraus ist per Metonymie die existentiale Lesart abgeleitet:

(118) ein Ort (ein Land) X ist durch einen Gegenstand Y gekennzeichnet

$\rightarrow$ Es gibt den Gegenstand $Y$ an dem Ort X.

Von diesem lokal-existentialen ausgehend haben kann nun wiederum das absolut-existentiale haben erklärt werden. Hier ist der Ortsbezug getilgt oder nur noch implizit gegeben, vgl. das Beispiel (119).

(119) Es hat von fast allem genug für fast alle.

(Variantenwb. 320)

Da existentiales haben für die weitere Argumentation keine Rolle spielt, soll an dieser Stelle nicht weiter darauf eingegangen werden. Dies wäre ein Gegenstand für eine eigene Untersuchung, die auch die sprachhistorischen Gegebenheiten angemessen $\mathrm{zu}$ berücksichtigen hätte. 
Wenn in der formal-semantischen Forschungsliteratur auf die „double nature of the verb have“ (Jensen/Vikner 1998: 113) bzw. auf das Nebeneinander von „existential have“ und ,the true verb have“ (Partee 2004: 289; vgl. Sæbø 2009: 1) abgehoben wird, läuft dies auf das Postulat zweier homonymer Verben ${ }^{1}$ haben und ${ }^{2}$ haben hinaus. Hier wurde dagegen ein Ansatz vertreten, demzufolge es ein einziges Verb haben mit einer unterspezifizierten Bedeutung ,INDEX“ gibt, welche die Basis für alle Lesarten des Verbs einschließlich der possessiven und der existentialen Verwendungen darstellt. Bevor auf haben-FVG im Rahmen dieser Hypothese eingegangen werden kann, ist noch einmal ihre grundsätzliche Erklärungskraft zu überprüfen.

\subsubsection{Vorhersagen auf der Basis der Index-Hypothese}

Die Validität der Hypothese, dass haben im Kern eine Indexrelation zwischen Objekt- und Subjekt-NP kodiert, ist vor allem an den Vorhersagen zu messen, die auf dieser Grundlage möglich sind. Wenn mit dieser These postuliert wird, dass die Objekt-NP einen Index für die Subjekt-NP darstellt, so müssen sich daraus relativ starke Beschränkungen für die Besetzung der Subjekt- bzw. Objektposition ergeben. Diese betreffen vor allem Nomina, die in einer Teil-Ganzes-Relation stehen. Hier scheint insofern eine Regularität zu bestehen, als das Ganze nur in der Subjektposition, ein dazu gehöriger Teil nur in der Objektposition lexikalisiert werden kann, vgl. (120)-(121).

(120) a. Der Hund hat einen Schwanz. (Teil $\rightarrow$ Ganzes)

b. ${ }^{\star}$ Der Schwanz hat einen Hund. (Ganzes $\rightarrow$ Teil)

a. Das Haus hat einen Balkon. (Teil $\rightarrow$ Ganzes)

b. ${ }^{\star}$ Der Balkon hat ein Haus. (Ganzes $\rightarrow$ Teil)

Diese Einschränkung ist auf der Basis der Indexikalitätshypothese vorhersagbar: Ein Ganzes ist in der Regel ein schlechter Index für eines seiner Teile, weil es komplex ist, d. h. mehrere Teile indizieren kann. Die Indexikalität eines Teils für das Ganze ist hingegen höher, weil ein Teil sich in der Regel nur auf ein (unmittelbares) Ganzes bezieht, so dass man mit Wilkins (1996: 281) generell festhalten kann: „Parts can index wholes, but wholes cannot index parts“. Eine Relation Ganzes $\rightarrow$ Teil verstößt somit gegen ein wesentlichen Merkmal von Indexikalität, nämlich die Exklusivität der Relation, vgl. dazu nochmals die eben genannten Beispiele (,!“ steht hier für ,verstößt gegen“, „!!“ für ,verstößt schwer gegen“ sowie „ $\sqrt{ }$ “ für ,verstößt nicht gegen'): 
(122) a. Der Hund hat einen Schwanz. (Teil $\rightarrow$ Ganzes) V $\sqrt{\text { Exklusivität }}$

b. ${ }^{\star}$ Der Schwanz hat einen Hund. (Ganzes $\rightarrow$ Teil) Exklusivität

a. Das Haus hat einen Balkon. (Teil $\rightarrow$ Ganzes) $\quad \sqrt{\text { Exklusivität }}$

b. ${ }^{\star}$ Der Balkon hat ein Haus. (Ganzes $\rightarrow$ Teil) ! Exklusivität

Eine weitere Auffälligkeit von haben, die auf der Basis anderer Beschreibungsansätze nicht zufriedenstellend vorhersagbar ist, betrifft die sog. „transitivity failure“ (Cruse 1986: 165-167; Croft/Cruse 2004: 158f.), die das Verb aufweist. ${ }^{131}$ So liegt in den Beispielen in (123) zwar durchaus eine Relation Teil $\rightarrow$ Ganzes vor, trotzdem ist nur (124a) kontextfrei voll akzeptabel, während (124b) und besonders (124c) spezifischer Kontextbedingungen bedürfen, um als akzeptabel zu gelten.
a. Die Hand hat fünf Finger.
b. \#Der Arm hat fünf Finger.
c. 'Der Oberkörper hat fünf Finger.

$\sqrt{ }$ Unmittelbarkeit

! Unmittelbarkeit

\section{!! Unmittelbarkeit}

Bei den Relationen zwischen Subjekt- und Objekt-NP, die in (125) gegeben sind, handelt es sich um Teil-Ganzes-Relationen, die in einer Verkettung miteinander verbunden sind:

$$
\text { Finger } \rightarrow \text { Hand } \rightarrow \text { Arm } \rightarrow \text { Oberkörper } \rightarrow \text { Körper }
$$

Die Akzeptabilität der Äußerungen in (124) hängt offenbar mit der Nähe bzw. Distanz zusammen, in der Teil und Ganzes stehen: Finger und Hand funktionieren mit haben besser als Finger und Arm bzw. Finger und Oberkörper. Hier ist somit das für Indexikalität kennzeichnende Merkmal Unmittelbarkeit bestimmend für das Akzeptabilitätsgefälle in (124): Der Schluss Finger $\rightarrow$ Hand ist leichter als der Schluss Finger $\rightarrow$ Arm oder Finger $\rightarrow$ Oberkörper, da von Finger unmittelbar auf Hand geschlossen werden, während der Schluss von Finger auf Arm/Oberkörper nur vermittelt über Finger $\rightarrow$ Hand und Hand $\rightarrow$ Arm möglich ist. Finger ist somit ein besserer Index für Hand als für Arm und Oberkörper. Die unterschiedliche Indexikalität der Lexeme Tür/Haus/Klinke erklärt auch das Verhalten von haben in

131 Langacker (1999: 49) erklärt dies damit, dass engl. have nur auf den Wissenshintergrund beziehbar ist, der für die Konzeptinterpretation unmittelbar relevant ist, d. h. den sog. ,immediate scope“. Weshalb dies so sein sollte, wird nicht ausgeführt. Dies müsste jedoch, wie hier vorgeschlagen, von der Bedeutung des Verbs her verständlich gemacht werden. 
dem bei Lyons (1977: 313), Cruse (1986: 156f.) und Croft/Cruse (2004: 158f.) diskutierten und jeweils unterschiedlich erklärten Fällen (126) und (127) (hier aufs Deutsche übertragen):

(126) a. . Das Haus hat eine Tür.

$\sqrt{ }$ Unmittelbarkeit

b. Die Tür hat eine Klinke.

$\sqrt{ }$ Unmittelbarkeit

c. ${ }^{\star}$ Das Haus hat eine Klinke.

! Unmittelbarkeit

a. Das Jackett hat Ärmel.

$\sqrt{ }$ Unmittelbarkeit

b. Die Ärmel haben Manschetten.

! Unmittelbarkeit

c. Das Jackett hat Manschetten.

$\sqrt{ }$ Unmittelbarkeit

Die Äußerung (127c) ist gegenüber (126c) akzeptabel, da die Manschetten als unmittelbare Teile des Ganzen Jackett konzeptualisiert werden können; Klinke ist dagegen nicht als unmittelbarer Teil von Haus identifizierbar. Dementsprechend ist ein Kompositum Jackettmanschette möglich (wie z. B. Hemdmanschette), eine Bildung ${ }^{\star}$ Hausklinke dagegen nicht. Eine Erklärung dafür, dass die mereologischen Verhältnisse in (126) und (127) voneinander abweichen, ist vermutlich in einer wahrnehmungspsychologischen Gegebenheit $\mathrm{zu}$ suchen: Ein Jackett ist kleiner und weniger komplex, so dass es in einem Zug auf seine Teile hin gescannt werden kann. Deshalb kann Manschette auch als unmittelbarer Teil von Jackett aufgefasst werden. Ein Haus ist dagegen im Normalfall wesentlich komplexer, größer und es weist zudem eine Unterscheidung zwischen Innen und Außen auf. Eine Ganzheit wie ,Haus' kann daher nur in mehreren aufeinander folgenden Schritten analysiert werden. ${ }^{132}$

Bei Teil-Ganzes-Relationen sind hinsichtlich der Indexikalitätshypothese einige interessante Einschränkungen festzustellen. In der von Gerstl/Pribbenow (1995: 879-883) eingeführten Alltagstheorie mereologischer Beziehungen wird zwischen folgenden Untertypen von Teil-Ganzes-Relationen unterschieden: Masse-Quantität (z. B. 100 Gramm von dem Mehl), Kollektion-Element (drei von fünf Äpfeln), Komplex-Komponente (Tür-Klinke). Lüdeling (1995: 90f.) weist auf der Basis dieser Unterscheidung darauf hin, dass nur die dritte der von

132 Nach Auffassung des Autors ist die Äußerung *Das Haus hat eine Klinke besser, wenn man sie auf ein Puppenhaus bezieht. In diesem Fall läge eine kleinere Einheit vor, die in einem bzw. mehreren kleinen Schritten analysiert werden kann. 
Gerstl/Pribbenow (1995) postulierten Teil-Ganzes-Relationen durch haben ausgedrückt werden kann (Die Tür hat eine Klinke). Für die Relationen Masse-Quantität und Kollektion-Element ist dies nicht möglich:

(128) a. 100 Gramm von dem Reis in der Pfanne

b. 'Der Reis in der Pfanne hat 100 Gramm.

a. Zwei der drei Äpfel im Korb

b. `Die drei Äpfel im Korb haben zwei.

(Beispiele nach Lüdeling 1995: 90f.)

Sieht man Indexikalität als Grundeigenschaft von haben an, so ist die Unvereinbarkeit der Relationstypen Masse-Quantität und Kollektion-Element mit dem Verb erklärbar. Von 100 Gramm Reis als einer Teilmenge kann nicht auf die genaue Gesamtquantität geschlossen werden, ebenso wenig kann auf der Basis der Angabe zwei Äpfeln im Korb darauf geschlossen werden, dass die Kollektion genau drei Äpfel umfasst. Dass jedoch gerade die Relation Komplex-Komponente mit haben funktioniert, ist genau darin begründet, dass unter den drei mereologischen Relationstypen der Klassifikation von Gerstl/Pribbenow (1995) allein der Typ Komplex-Komponente indexikalisch ist, und zwar insofern, als von der Komponente direkt auf den Komplex geschlossen werden kann. Dies gilt allerdings, wie oben gezeigt, nur für den jeweils unmittelbar höheren Komplex, da Indexikalität keine transitive Relation darstellt.

\subsubsection{Haben und die Beschränkungen in der Kombinierbarkeit}

\subsubsection{FVG mit Gefühlsnomina}

Eine besondere Herausforderung für die Analyse von haben-FVG stellen Nomina dar, die einen emotionalen Zustand beschreiben. ${ }^{133}$ Hier liegt, wie zu Beginn dieses Kapitels bereits angedeutet, eine auf den ersten Blick paradoxe Situation vor.

133 Als Test für die Zugehörigkeit eines Nomens zur semantischen Klasse, emotionaler Zustand sollen hier die Kombinierbarkeit mit empfinden (z. B. Hass empfinden), das mögliche Vorkommen als Genitivattribut zu Gefühl (Gefühl des Hasses) sowie die Einsetzbarkeit in Formulierungen wie $X$ überkam mich gelten. Eine Abgrenzung gegenüber den Nomina, die mentale Einstellungen bezeichnen, s. u. (153), ist gleichwohl nicht immer möglich. 
Mit einer Reihe von Gefühlsnomina kann haben ohne weiteres kombiniert werden, vgl. (130). ${ }^{134}$

(130) (einen) Abscheu, Angst, Durst, Ekel, die Empfindung, Freude, Furcht, das/ein Gefühl, einen Grusel, einen Hass, Hemmungen, Hunger, Kummer, Langeweile, gute/schlechte Laune, Lust, Mitleid, Panik, Skrupel, Scheu, Spaß (?), Widerwillen, einen Zorn, eine Wut ${ }^{135}$

Zahlreiche andere Gefühlsnomina sind in einer Verbindung mit haben jedoch nicht möglich, vgl. die Liste in (131).

(131) Ärger ${ }^{136}$, Ausgelassenheit, Beschämung, Begeisterung, Bestürzung, Beglückung, Betretenheit, Beunruhigung, Erregung, Erschrecken, Erstaunen, Entzücken, Enttäuschung, Euphorie, Fröhlichkeit, Frustration, Irritation, Kränkung, Leid, Niedergeschlagenheit, Rührung, Schaudern, Trauer, Traurigkeit, Scham, Schmerz (Sing.), Unmut, Verärgerung, Verbitterung, Verlegenheit, Verwunderung, Verwirrung, Verzweiflung

Auffallend ist, dass unter den Nomina in (130), die mit haben kombinierbar sind, keine deadjektivischen Bildungen zu finden sind. Dies hat als Konsequenz, dass semantisch sehr eng verwandte Nomina sich - offensichtlich ihrer morphologischen Struktur entsprechend - in haben-FVG entgegengesetzt verhalten:

(132) a. Kummer haben vs. *Traurigkeit haben

b. Freude, Spaß haben vs. *Ausgelassenheit, *Fröhlichkeit haben

Die Gruppe der nicht FVG-fähigen Gefühlsnomina in (131) dagegen setzt sich zu einem großen Teil aus deadjektivischen Nomina (Ausgelassenheit, Traurigkeit) sowie Derivationen auf -ung zusammen (Kränkung, Begeisterung). Letztere sind

134 In der Liste werden nur die einschlägigen Nomina lediglich aufgezählt; auf spezifische Eigenschaften - Artikelfähigkeit, syntaktischer Anschluss - wird hier nicht eingegangen (lediglich, wenn das Nomen nicht artikellos steht, wird der entsprechende Artikel genannt). Die Liste umfasst auch umgangssprachliche Verbindungen wie einen Ekel haben vor, einen Hass haben auf.

135 Unter der Kategorie „mentale Zustände“ werden Gefühle, Erkenntniszustände sowie innere Einstellungen gegenüber Sachverhalten gefasst.

136 Ärger haben kommt in der Bedeutung ,sich ärgern' (um die es hier allein geht) nicht vor. Es ist vielmehr idiomatisiert und steht für ,Unannehmlichkeiten erfahren`. 
nach Bierwisch (1989: 60f.) aufgrund ihrer Argumentstruktur als Ableitungen der passivischen Verbversionen anzusehen:

$$
\text { Aufregung, Begeisterung ... < aufgeregt, begeistert sein ... }
$$

Als Begründung führt Bierwisch an, dass sowohl in der Nominalisierung als auch beim Passivstamm nur eine Theta-Rolle erlaubt ist, während die Rolle, die die kausative Komponente enthält, fehlt; vgl. (134).
a. Peters Begeisterung
b. *die Begeisterung des Films

In diesem Sinne konstatieren auch Fleischer/Barz (2012: 229) eine enge „Beziehung zwischen -ung-Derivat und Partizip II“. Dass ein zufälliges morphologisches Faktum für die Selektion der Nomina in haben-FVG ausschlaggebend sein sollte, erscheint vor dem Hintergrund der bei anderen Verben herausgearbeiteten Regularitäten freilich wenig plausibel. Eher ist hinter der morphologischen Restriktion eine semantische gesteuerte Beschränkung zu vermuten, wie sie für die anderen hier untersuchten FVG plausibel gemacht werden konnte.

Diese semantische Motivation für den Ausschluss deadjektivischer bzw. vom Partizip II gebildeter Nomina wird in einer Eigenschaft zu suchen sein, die für adjektivische Attribuierung grundlegend ist: Bis auf wenige Ausnahmen (wie z. B. ehemalig oder Gradajektive wie groß, klein) sind Adjektive intersektiv (vgl. Heim/Kratzer 1998: 65; aus kognitiv-linguistischer Sicht Sweetser 1999: 137-140). Der Begriff ,intersektiv“ bezieht sich darauf, dass die semantische Komposition von Nomen und Adjektiv als Schnittmenge ihrer jeweiligen Extensionen beschrieben werden kann. So denotiert fröhlicher Junge die Menge der Individuen, die sowohl fröhlich als auch Junge sind (d. h. expliziter formuliert: die Denotation von $\lambda x$ [fröhlich' (x) \& Junge' (x)] ist der Durchschnitt der Denotationen von $\lambda$ $\mathrm{x}$ [fröhlich' (x)] und $\lambda x$ [Junge' (x)]). Eine Phrase wie in (135a) ist daher die Summe der Extensionen von (135b) und (135c).

(135) a. Peter ist ein fröhlicher Junge.

b. Peter ist ein Junge.

c. Peter ist fröhlich. 
Eine Indexrelation kann, wie oben gezeigt, nur funktionieren, wenn Index und Indicatum unterscheidbar sind; vgl. dazu nochmals die entsprechende Formulierung aus Abschnitt 5.5.4:

(ii)' Unterscheidbarkeit: Die involvierten Konzepte dürfen keine identische Extension aufweisen, sondern müssen als distinkte Entitäten wahrnehmbar sein. (Die Fußspur muss erst von Fuß unterschieden werden können, der Rauch muss getrennt vom Feuer wahrnehmbar sein, damit eine Indexfunktion etabliert werden kann.)

Die intersektive Adjektivattribuierung erfüllt die für Indexikalität wesentliche Bedingung der Unterscheidbarkeit nicht: Innerhalb der Extension fröhlicher Junge gibt es kein Element, auf das die Eigenschaften fröhlich sein und Junge sein nicht gleichzeitig zutreffen. ${ }^{137}$

Eine indirekte Bestätigung dieses Zusammenhangs ergibt sich daraus, dass einzelne haben-Prädikationen mit deadjektivischen Nomina akzeptabler werden, sobald sie in Verbindung mit einem lokalen Adjunkt auftreten, vgl. (136).

(136) Ich habe Traurigkeit in meinem Herzen.

In (136) wird die Eigenschaft Traurigkeit nicht mit der gesamten Denotation von Ich gleichgesetzt, sondern bleibt auf die Subdomäne mein Herz beschränkt (Langacker 1987: 272f. würde hier von ,active zone‘ sprechen). Da die Eigenschaft Traurigkeit von Ich unterscheidbar ist, kann indexikalisches haben verwendet werden.

Der Unverträglichkeit von haben mit der intersektiven Semantik von Adjektiven erklärt wahrscheinlich auch das zunächst völlig arbiträr erscheinende Verhalten des Substantivs Freude. Dieses kann nur dann mit haben stehen, wenn es in einer von an oder mit regierten PP vorkommt; Freude über $X$ ist jedoch nicht mit haben vereinbar.

(137) a. Ich habe (meine) Freude an/mit dem Geschenk.

b. *Ich habe Freude über das Geschenk.

$137 \mathrm{Zu}$ erwarten wäre, dass Nominalisierungen auf der Basis nicht-intersektiver Adjektive mit haben kombinierbar sind. Das dies offensichtlich nicht der Fall ist, hat seinen schlichten Grund darin, dass nicht-intersektive Adjektive nicht nominalisierbar sind: ${ }^{\star}$ Ehemaligkeit, \#Größe (nur in intersektiver Lesart, vgl. dazu auch die weiteren Ausführungen). 
Eine Erklärung für die Inkompatibilität von Freude über $X$ und haben ist darin zu sehen, dass dieses in Relation zu passivischem erfreut sein über steht. So sind (138a) und (138b) referenzidentisch.

(138) a. Meine Freude über den Besuch

b. Ich bin erfreut über den Besuch.

Da erfreut über als intersektiv gelten kann - ein über den Besuch wenig erfreuter Junge ist die Schnittmenge aller Individuen, die wenig erfreut über den Besuch und gleichzeitig Junge sind -, ist verständlich weshalb auch Freude über keine Verbindung mit haben eingehen kann. Für Freude an ist keine Beziehung zu einem intersektiven Adjektiv erfreut konstruierbar, vgl. (139). Daher ist Freude haben an etwas vollkommen akzeptabel.

(139) a. Meine Freude an dem Besuch

b. ${ }^{\star}$ Ich bin erfreut an dem Besuch.

c. Ich habe Freude an dem Besuch.

Eine weitere, bisher noch nicht angesprochene Beschränkung für die Kombinationsmöglichkeiten von haben mit Gefühlsnomina ergibt sich aus den aktionalen Eigenschaften des Verbs, genauer gesagt aus seiner Festlegung auf den Ereignistyp ,Zustand‘, s. o. (113b). Vor diesem Hintergrund erscheint es plausibel, dass Nomina wie Schaudern, Erschaudern, Erschrecken, Entsetzen in einem haben-FVG nicht vorkommen. Hier liegt tendenziell ein punktuelles, mindestens aber ein zeitlich begrenztes Ereignis vor, so dass keine Vereinbarkeit mit dem ,State“ haben gegeben ist.

Bei einzelnen Gefühlsnomina ist freilich keine übergreifende Erklärung möglich bzw. überhaupt keine fundierte Hypothese formulierbar, die deren Fehlen in haben-FVG erklären könnte. Dies betrifft insbesondere Trauer und Scham.

$$
{ }^{\star} \text { Der Junge hatte Trauer/Scham/Leid/Schmerz. }
$$

Ein FVG kann in diesen Fällen teilweise mit empfinden gebildet werden. Die Gründe für das Kombinationsverhalten sind nicht ganz klar. Bei Trauer und Scham könnte es daran liegen, dass hier keine, echten' Gefühlsnomina vorliegen: Trauer ist nicht allein ein Gefühl, sondern auch eine ritualisierte Handlung; Scham ist mit Beschämung gleichzusetzen, d. h. mit adjektivischem beschämt sein 
als Basis, und zudem ist es historisch gesehen ebenfalls kein genuines Gefühlswort, da es ursprünglich ,Schande“ bedeutet - ob ein historisches Argument hier weiterhilft, sei indes dahingestellt. Wahrscheinlich ist hier, ähnlich wie im Fall von ${ }^{\star}$ Befürwortung geben vs. Zustimmung geben, ein syntaktischer Grund ausschlaggebend: Im Gegensatz zum einfachen Verb sich einer Sache schämen bzw. sich für etwas schämen ist es für das Nomen Scham schwierig, eine passende syntaktische Anschlussstelle zu eröffnen. Es kann weder mit einem Genitivattribut noch mit einer für-PP kombiniert werden. Lediglich umgangssprachliches Scham wegen wäre denkbar. Die Fügungen ${ }^{\star}$ Leid haben bzw. * Leiden haben als Entsprechungen zum einfachen Verb leiden (an, unter etwas) sind aus ähnlichen Gründen unpassend. Leiden ,Schmerz empfinden' fordert eine Ergänzung mit unter, ?Leid unter ist jedoch fraglich.

(141) a. Er leidet unter dem Lehrer.

b. ${ }^{*}$ Er hat Leid/Leiden unter dem Lehrer.

An einer Krankheit leiden ,krank sein“ ist wiederum selbst Kandidat für ein FVG, da das relationale Nomen Krankheit bereits die lexikalische Information der gesamten VP enthält. Dass auf der Basis eines FVG ein weiteres FVG gebildet werden kann, erscheint nicht naheliegend (auch wenn es sicher nicht prinzipiell ausgeschlossen werden kann). ${ }^{\star}$ Schmerz haben (im Singular) schließlich ist als Pendant zu jmdn. schmerzt etwas ebenfalls aus syntaktischen Gründen unpassend: Der Stimulus, der in der verbalen Konstruktion im Subjekt realisiert wird, müsste in einem FVG an das Nomen anschließbar sein. Hier fehlt aber ebenfalls eine überzeugende Anschlussmöglichkeit: 'Schmerz von, ?Schmerz durch X.

\subsubsection{FVG mit Individuenprädikaten}

Vor dem Hintergrund der bisherigen Ausführungen zeigen Nomina, die ein Individuenprädikat ausdrücken, ein auf den ersten Blick nicht zu erwartendes Muster (zur Unterscheidung ,individual-level-predicates“ vs. „stage-level-predicates“ grundlegend Carlson 1977 sowie Kratzer 1995). HabenPrädikationen sind in einigen Fällen nämlich durchaus auch mit deadjektivischen Nomina möglich, vgl. (142).

(142) Das Mädchen hat Schlagfertigkeit/Intelligenz/Größe.

Die oben postulierte Regel, der zufolge nicht-deadjektivische Nomina für habenFVG ungeeignet sind, scheint hier somit außer Kraft gesetzt zu sein. Als Erklärung 
ließe sich anführen, dass es sich bei Individuenprädikaten um Eigenschaften handelt, die per Definition für die gesamte Existenzdauer eines Individuums gültig sind. Insbesondere belebte bzw. menschliche Eigenschaftsträger zeichnen sich jedoch dadurch aus, dass sie durch mehrere Eigenschaften charakterisiert sind - kein Individuum ist sein Leben lang ausschließlich durch ein einziges Prädikat bestimmt. Dieses Weltwissen könnte durchaus eine Motivation für die Kombinierbarkeit von Individuenprädikaten mit haben darstellen. Als Erklärung reicht dies aber kaum aus, da z. B. auch fröhlich eine Lesart als Individuenprädikat hat (Peter ist ein fröhlicher Junge), dieses aber, wie gezeigt, keine Ableitungsbasis für ein FVG-Nomen bildet.

Auffallend an den Nomina in (142) ist freilich, dass sie durchweg charakterliche Qualitäten versprachlichen, die vor allem für den gesellschaftlichen Umgang und auch für das gesellschaftliche Ansehen einer Person entscheidend sind. Unmittelbar greifbare physische Eigenschaften finden sich hier nicht. Auch Temperamente oder sonstige mentale Dispositionen (Fröhlichkeit, Pessimismus) fehlen in der Aufzählung. Das Besondere an dieser Gruppe kann an der Eigenschaft ,groß‘ deutlich gemacht werden. Eine haben-Prädikation ist in diesem Fall nur dann akzeptabel, wenn sie auf ein charakterliches Merkmal ,groß‘ bezogen wird, vgl. (143a). Als Beschreibung eines körperlichen Merkmals ,groß gewachsen' kommt ein haben-FVG nicht in Betracht, wie (143b) belegt.
a. Das Mädchen hat Größe. (Charakterzug)
b. `Das Mädchen hat Größe. (,Das Mädchen ist groß gewachsen`)

Dies lässt den Schluss zu, dass in der Auflistung in (142) eben nicht die Kernmerkmale einer menschlichen Persönlichkeit (Größe, Geschlecht, Alter u. ä.) enthalten sind, sondern ganz überwiegend Eigenschaften, die jemandem aufgrund eines über längere Zeit wahrnehmbaren Verhaltens von Seiten des sozialen Umfelds zugesprochen werden; diese Eigenschaften können einer Person aber auch relativ leicht wieder abgesprochen werden, wesentlich leichter als die persönlichkeitskonstituierenden Kernmerkmale. Diese ,Tugenden“ sind also bei weitem nicht so eng mit dem Eigenschaftsträger verbunden wie physische Eigenschaften. Die Unterscheidbarkeit von Index und Indicatum, von Eigenschaft und Eigenschaftsträger ist bei einem Konzept ,moralische Größe‘ somit deutlicher sichtbar als bei körperlicher Größe.

Erwähnenswert ist in diesem Zusammenhang, dass eine Äußerung wie (144), obwohl sie ein physisches Merkmal benennt, gleichwohl als korrekt gelten darf:

(144) Das Mädchen hat eine Größe von 1,70. 
Hierauf soll weiter unten noch eingegangen werden.

Bei der Klärung der Frage, weshalb bei den FVG in (142) auch deadjektivische Nomina in Erscheinung treten, obwohl sie doch nach dem oben Gesagten nicht vorkommen dürften, ist zu berücksichtigen, dass die betreffenden Prädikationen typischerweise in Reihungen belegt sind. So ergibt eine COSMAS-Suche nach der Wortfolge haben + Intelligenz 37 einschlägige Textstellen; ${ }^{138}$ in 34 dieser Treffer wird Intelligenz jedoch als eine unter mehreren Eigenschaften genannt, vgl. als Beispiel die Äußerung (145).

(145) Denn der Organisator und Propagandist der Nazi-Bewegung hatte Intelligenz, Sprachgewalt, ein Gespür für Stimmungen, Skrupellosigkeit, Zynismus und Fanatismus. (Nürnberger Zeitung, 13.04.2005; Zugriff 06.04.2021)

Die Aufzählung mehrerer Charaktereigenschaften macht deutlich, dass das Subjekt nicht mit einer dieser Eigenschaften zusammenfällt. Damit ist die Unterscheidbarkeit von Index und Indicatum gewährleistet.

Lediglich in drei Treffern steht Intelligenz haben ohne weitere Eigenschaft, vgl. stellvertretend für die anderen den Beleg (146).

(146) [...] Sein Reichtum bedeutet ihm wenig: „Geld ist für mich nichts als Energie.“ Die wirkliche Philosophie stecke in der Natur: „Pflanzen haben Intelligenz“. (Kleine Zeitung, 17.01.2000, Ressort: Wirtschaft; Zugriff 06.04.2021)

In diesem Fall wird ein Kontrast zu einer Standardannahme über Pflanzen formuliert: Eine Eigenschaft, von der man in der Regel nicht annimmt, dass sie auf den Eigenschaftsträger zutrifft, wird diesem gegen jede Erwartung explizit zugesprochen (die beiden anderen Belege aus der Korpusrecherche sind vergleichbar). Die Unterscheidbarkeit von Eigenschaft und Eigenschaftsträger - hier also ,intelligent sein' und ,Pflanze“ - wird damit als Default vorausgesetzt.

Äußerungen wie $X$ hat Intelligenz, aber auch $X$ hat Länge gewinnen zudem deutlich an Akzeptanz, wenn das Nomen durch ein Adjektivattribut modifiziert wird, das ein Maß oder einen Grad angibt, vgl. (147)-(148).

138 Suche nach „\&haben Intelligenz“ (direkt hintereinander), insgesamt 105 Treffer, davon 37 einschlägig (letzter Zugriff 06.04.2021). 
(147) a. \#Der Junge hat Intelligenz. ${ }^{139}$

b. Der Junge hat eine bemerkenswerte Intelligenz.

(148) a. `Das Buch hat Länge. (Für ,Das Buch ist lang.')

b. Das Buch hat eine ausreichende Länge.

Die NP bemerkenswerte Intelligenz/ausreichende Länge impliziert einen Vergleich gegenüber einem als durchschnittlich vorausgesetzten Maß an Intelligenz bzw. Länge („the contextually salient standard“ bei Heim/Kratzer 1998: 71). In diesem Fall wird der Referent mit anderen Referenten in Relation gesetzt und hinsichtlich der Ausprägung einer bestimmten Eigenschaft verglichen. Die Vergleichsoperation macht es nötig, in einem ersten Schritt zwei Referenten sowie eine Eigenschaft einzuführen, die dann in einem folgenden Schritt miteinander in Beziehung gesetzt werden. Mengentheoretisch formuliert, wird bei einem Vergleich die Schnittmenge des Referenten X mit einer Eigenschaft P mit der Schnittmenge von $\mathrm{Y}$ und $\mathrm{P}$ abgeglichen. Wenn $\mathrm{X}$ intelligenter als $\mathrm{Y}$ ist, dann ist der Anteil, den X an der Eigenschaft Intelligenz hat, größer als der entsprechende Anteil von Y. Dies impliziert aber auch, dass die Entitäten X und Y nicht zur Gänze mit der Eigenschaft $\mathrm{P}$ gleichzusetzen sind. Damit ist auch hier das Indexikalitätsmerkmal der Unterscheidbarkeit gegeben. ${ }^{140}$

Dieser Zusammenhang bestätigt sich, wenn man explizite Vergleiche mit haben wie in (149) heranzieht. Auch hier macht das Gegebensein einer Vergleichsoperation die Verwendung von haben akzeptabler:

(149) a. \#Der Junge hat Intelligenz.

b. Der Junge hat eine Intelligenz wie Einstein.

Einen impliziten Vergleich kann man übrigens auch für das eben erwähnte $X$ hat eine Größe von $Y$ in (144) voraussetzen; diese Formulierung setzt die Existenz einer Menge potentieller Körpermaße im Diskursuniversum voraus, aus der genau dieses eine gewählt wird.

Ein Vergleich mit bzw. einen Kontrast zu einem Normalmaß findet sich auch bei einer anderen haben-Fügungen, die freilich nicht als Individuen-, sondern

139 Die Äußerung ist akzeptabel, freilich nur, wenn man eine inhaltliche Hervorhebung annimmt. Aus diesem Grund wird sie mit „\#“ markiert.

140 Zum Artikelgebrauch in diesen Fügungen s. Kapitel 6.3. 
eher als Stadienprädikat zu klassifizieren ist. Es handelt sich um die Verbindungen in (150).

(150) Der Student hatte den Mut/die Frechheit, zu protestieren.

(150) ist als eine Eigenschaftszuschreibung , $\mathrm{X}$ ist so mutig/frech, dass $\mathrm{X}$ in einem bestimmten Augenblick P tut' zu paraphrasieren; diese Fügung drückt die besonders starke Ausprägung eines Merkmals aus, die dann in einer bestimmten Handlung des Eigenschaftsträgers mündet. Ein Abgleich mit einem Normalmaß ist also auch hier implizit gegeben.

Die hier vertretene Hypothese, dass die Akzeptabilität eines Nomens in einer haben-Fügung von dessen Indexikalität abhängt, lässt freilich noch die Frage offen, weshalb nominale Individuenprädikate wie Feigheit, Kleinlichkeit, Dummheit nicht oder nicht so gut in die N-Position eines haben-FVG eingesetzt werden können, obwohl deren jeweiliges positives Gegenstück vollkommen akzeptabel ist, wie sich an (151a) und (152a) vs. (151b) und (152b) zeigen lässt.

(151) a. Ich habe Mut.

b. ${ }^{\star}$ Ich habe Feigheit.

(152) a. Ich habe die Fähigkeit, mehrere Dinge gleichzeitig zu tun.

b. `Ich habe die Unfähigkeit, mehrere Dinge gleichzeitig zu tun.

Eine Erklärung hierfür ist möglicherweise darin zu suchen, dass Feigheit/Dummheit/Unfähigkeit primär als Negation ihrer positiven Entsprechungen, d. h. als Abwesenheit von Mut/Intelligenz/Fähigkeit konzeptualisiert sind. Der Schluss von einer nicht-vorhandenen, als ungültig assertierten Eigenschaft auf einen Eigenschaftsträger ist sicher wesentlich problematischer als ein Schluss, der auf einer assertierten Eigenschaft basiert. Die Indexikalitätshypothese bietet somit auch hier einen Interpretationsansatz.

\subsubsection{FVG mit Kognitions- und Einstellungsnomina}

Eine umfangreiche Gruppe von FVG mit haben bilden neben den Ausdrücken für emotionale Zustände auch Verbindungen, die innere Einstellungen oder kognitive Zustände versprachlichen; vgl. dazu die Liste in (153):

(153) Achtung (vor), die/eine Ahnung, das/ein Bestreben, Bedenken, (den/einen) Gedanken, Gefallen, den/einen Einfall, den/einen Eindruck, die/eine Erkenntnis, die/eine Erwartung, Genugtuung, den 
Glauben, (einen) Hintergedanken, die/eine Hoffnung, die/eine Idee, die/eine Intuition, die/eine Meinung, Misstrauen, Mühe, Respekt, Skrupel, den/einen Traum, die Überzeugung, den/einen Verdacht, die/eine Vermutung, (das) Vertrauen, Vorbehalte, die/eine Vorstellung, (Possessivpron.) Zweifel

Wie für die oben behandelten Nomina für Emotionen gilt auch für die Gruppe in (153), dass mentale Zustände sowie Gefühlsausdrücke stets auf einen Experiencer verweisen. Sie sind daher mit der Indexikalität von haben vereinbar und können in einem entsprechenden FVG auftreten.

Im Hinblick auf die Kompatibilität mit haben sind hier allenfalls die FVG einen Einfall/Geistesblitz haben erläuterungsbedürftig. Diese Fügungen kodieren ein punktuelles Ereignis und stehen daher im Widerspruch zum Zustandscharakter des Verbs haben, s. o. (113b). Dass sie trotzdem in der Liste vorkommen, mag mit einer Analogie zu begründen sein: Da Erkenntnis haben vorkommt, können auch Nomina, die eine plötzlich eintretende Erkenntnis versprachlichen, in der Fügung realisiert werden. Dieses Problem könnte aber möglicherweise auch als Hinweis darauf gesehen werden, dass der Zustandscharakter für haben gar nicht so zentral ist. Für Indexikalität ist eine längere Dauer der Relation jedenfalls nicht zwingend: Die Relation muss lediglich solange existieren, damit erfolgreich vom Index auf das Indicatum geschlossen werden kann.

$\mathrm{Zu}$ den Nomina in (153) ist anzumerken, dass für eine Teilmenge auch eine Verbindung mit besitzen oder verfügen über möglich ist, so dass in diesen Fällen eher von einer Extension der Besitzlesart von haben auszugehen ist:

(154) Achtung, Bewusstsein, Erkenntnis, (die) Gewissheit, Gespür, Bewusstsein, Einstellung, ein Interesse, Hochachtung, Kenntnis, (das) Verständnis, Vorliebe, Neigung, Leidenschaft

Die Zuordnung zu indexikalischem bzw. possessivem haben ist partiell offenbar davon abhängig, ob die Einstellung bzw. der kognitive Zustand permanent oder temporär ist. So ist plausibel, dass Substantive wie in (155), die sich auf in der Regel zeitlich begrenzte Bewusstseinsinhalte beziehen, nicht mit besitzen, sondern nur mit haben kombinierbar sind.

(155) Gedanken, Meinung, Traum, Einfall, Eindruck, Erkenntnis (anders Plur.), Idee, Erwartung 
Da besitzen gegenüber haben den Aspekt der dauerhaften Kontrolle einen Possessors über ein Possessum stärker betont, ist dieser Befund wenig überraschend.

Auch epistemische Einstellungen zu einem Sachverhalt, die keine Gewissheit über dessen Gegebensein enthalten, sind tendenziell nicht mit besitzen verbindbar. Dieses ist hier, wie (156) zeigt, gegenüber haben jedenfalls die schlechtere Variante.

(156) ('Er besitzt) Glauben, Vermutung, Verdacht, Zweifel.

Hinzu kommt, dass auch negative Eigenschaften nicht gut mit besitzen kombiniert werden können.

(157) ('Er besitzt) Vorbehalte, Misstrauen, Bedenken.

Da man negative Eigenschaftsausprägungen schlecht besitzen, d. h. über sie verfügen kann, vgl. (158), ist es plausibel, dass haben in diesen Fällen die bessere Wahl ist.

(158) a. *Ich besitze eine Schuld von 100 Euro bei der Bank.

b. Ich besitze ein Guthaben von 100 Euro bei der Bank.

Haben ist gegenüber besitzen semantisch deutlich unspezifischer; dementsprechend sind auch die Kombinationsmöglichkeiten von haben weniger eingeschränkt als die von besitzen.

\subsection{Fazit}

Die Ausführungen zu den FVG mit bringen, machen, geben und haben konnten zeigen, dass die Selektion der Ereignisnomina alles andere als arbiträr ist. Als der wichtigste Faktor, der die Wahl der Nomina beschränkt, hat sich die Bedeutung des FV erwiesen: In FVG können nur solche Nomina auftreten, die mit der semantischen Grundstruktur des Verbs kompatibel sind. Ob ein Nomen in einem FVG vorkommen kann, hängt zudem auch immer davon ab, ob das sich daraus ergebende FVG syntaktisch wohlgeformt ist, was sich u. a. an dem Gegensatz zwischen ${ }^{\star}$ Befürwortung geben und Zustimmung geben plausibel machen ließ.

Auf der Basis der Feststellung, dass die Selektion der Nomina regelhaft ist, kann für die betreffenden FVG auch eine bessere Einordnung hinsichtlich ihrer Stellung zwischen Grammatik und Lexikon erreicht werden: Die einzelnen FVG 
sind nicht notwendigerweise als je eigener Phraseologismus im Lexikon gespeichert, und es muss auch keine beliebig zusammengestellte Liste von Nomina angenommen werden, die mit den einzelnen FV korrespondieren (Sailer 2003). Auch die These eines durch ein Netz von Familienähnlichkeiten zusammengehaltenen Idiomverbunds (Zeschel 2008) erweist sich vor dem Hintergrund der hier erzielten Ergebnisse als nicht ausreichend. Vielmehr ist von einer NomenVerb-Konstruktion auszugehen, die insofern grundsätzlich regelhaft ist, als die Selektion der Nomina klaren semantischen Restriktionen unterliegt.

Die hier ermittelten Befunde werfen indes auch Probleme auf. So ist ein offensichtlicher Widerspruch aufzuklären: In Kapitel 3 wurde dargelegt, dass das FV keinen Beitrag zur lexikalischen Gesamtbedeutung der Nomen-Verb-Verbindung beisteuert. Insofern kann es als semantisch leer gelten. In Kapitel 5 wurde jedoch zu zeigen versucht, dass das Verb die Selektion der Nomina bestimmt. Ein semantisch leeres Verb kann freilich keine Selektionsbeschränkungen ausüben (und es kann auch nicht von einem anderen sprachlichen Ausdruck selektiert werden). Entweder muss also eine der beiden bisher aufgestellten Hypothesen ,Das FV ist semantisch leer' bzw. , Das FV schränkt die Auswahl der Nomina ein“ falsch sein oder es bietet sich ein dritter Lösungsweg an, der zwischen den Hypothesen zu vermitteln vermag. Da die eben angesprochene Frage nach den Lexikoneinträgen auf das Engste mit der Frage nach dem semantisch leeren, aber in der Selektion wirksamen FV zusammenhängt, seien beide Themenkomplexe gemeinsam in Kapitel 8 behandelt. 